\title{
Sets of numbers from complex networks perspective
}

\author{
PHD DISSERTATION
}

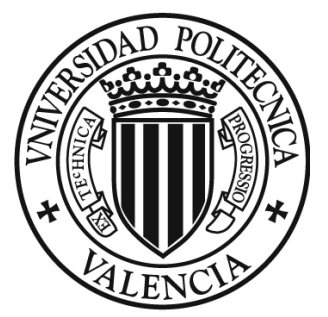

\author{
Author \\ Pedro Antonio Solares Hernández
}

\author{
Advisors \\ Dr. J. Alberto Conejero \\ Dr. Miguel A. García March
}

Valencia, September 2021 



\section{Dedicatorias}

A Dios, por permitirme llegar a este punto tan importante del desarrollo de mi trayectoria académica.

A mis directores de tesis, Dr. J. Alberto Conejero Casares y Dr. Miguel Ángel García-March, sin los cuáles no habría sido posible llegar a este sueño cumplido.

A los padrinos y contribuyentes de la tesis, Ministerio de Educación Superior Ciencia y Tecnología de la República Dominicana, Fundación Casa Brugal, Fundación Proyecto Escuela Hoy Inc., Fundación para el Avance de las Matemáticas, Superintendencia de Salud y Riesgos Laborales de la República Dominicana, y Francisco D'óleo Ramírez.

A mis padres, Pedro A. Solares y Rafaela Hernández, por haber creído en mi. Por darme lo mejor que pudieron cada día. El amor que me dieron, me acompanará siempre. Les dedico este logro y espero que siempre se sientan orgullosos de mí.

A mi esposa, Miranda Granero Terol, por darme el apoyo emocional que siempre necesité y hacerme sentir tan especial.

A mis hermanos, Rafael, Evelyn, Raúl, Yina, Willy y Daniel, en quienes me apoyé en los días en los que me faltaron algunas sonrisas y motivaciones.

A mi madrina, Dra. Maricarmen Álvarez Carrera, por siempre mantenerme ilusionado para lograr esta meta. Este logro también es de ella.

A mis suegros, Domingo Granero y Xelo Terol, por hacerme parte de sus vidas durante este maravilloso proceso de crecimiento profesional.

A mi mejor amigo, Carlos M. Infante Hernández, por siempre decirme que lo podía lograr.

A mis compañeros de investigación, Fernando A. Manzano Aybar, y Francisco Javier Pérez-Benito, en quienes me apoyé para iniciar el maravilloso viaje del aprendizaje de la programación computacional.

Finalmente, a todos aquellos que se sientan aludidos, muchísimas gracias. 



\section{Table of Contents}

Dedicatorias .................................. vii

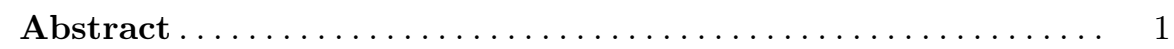

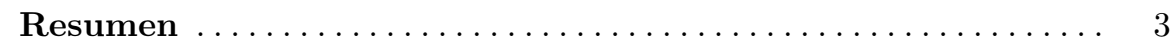

Resum ......................................... 5

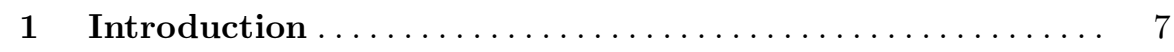

1.1 Examples of application of complex networks ........... 8

1.2 A perspective on divisibility networks $\ldots \ldots \ldots \ldots \ldots \ldots$

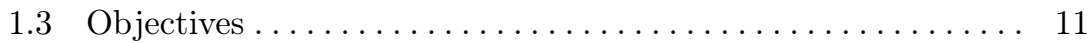

1.4 Preliminaries. . .................................. 12

1.4 .1 What is a network?. . . . . . . . . . . . . . . . . . . . . 12

$1.4 .2 \quad$ Basic notation and network measures . . . . . . . . . . 12

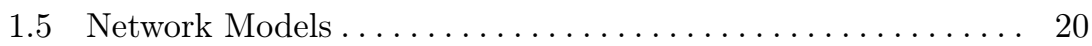

1.5 .1 Random Networks $\ldots \ldots \ldots \ldots \ldots \ldots \ldots \ldots \ldots \ldots \ldots$

$1.5 .2 \quad$ Small world networks $\ldots \ldots \ldots \ldots \ldots \ldots \ldots \ldots \ldots \ldots \ldots$

$1.5 .3 \quad$ Scale-Free Networks $\ldots . . . \ldots \ldots \ldots \ldots \ldots \ldots$. . . . . . . . . . 31

1.5.4 Hierarchical networks $\ldots \ldots \ldots \ldots \ldots \ldots \ldots \ldots \ldots . \ldots \ldots$

1.6 Network in number theory $\ldots \ldots \ldots \ldots \ldots \ldots \ldots \ldots \ldots \ldots \ldots$

1.6 .1 Divisibility networks $\ldots \ldots \ldots \ldots \ldots \ldots \ldots \ldots \ldots \ldots . \ldots \ldots$

1.6.2 Network analyses of conjectures in number theory .... 43

1.6.3 Networks based on Fibonacci numbers . ........... 46

2 Divisibility patterns within Pascal divisibility networks (Journal article) $\ldots \ldots \ldots \ldots \ldots \ldots \ldots \ldots \ldots \ldots \ldots \ldots \ldots \ldots \ldots$

Abstract ......................................... 49

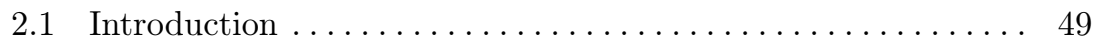

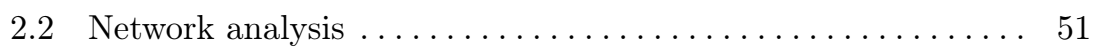

$2.2 .1 \quad$ Degree distribution $\ldots \ldots \ldots \ldots \ldots \ldots \ldots \ldots \ldots \ldots \ldots \ldots$

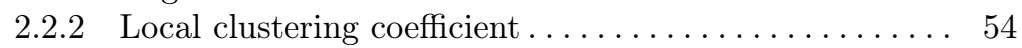

$2.2 .3 \quad$ Global clustering coefficient . . . . . . . . . . . . . . . . 57

2.2 .4 Assortative coefficient $\ldots \ldots \ldots \ldots \ldots \ldots \ldots \ldots \ldots \ldots$

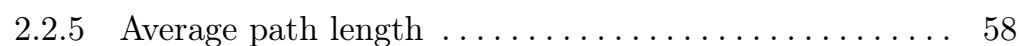




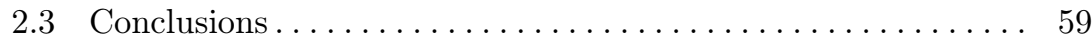

3 Divisibility networks of the rational numbers in the unit

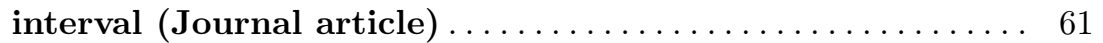

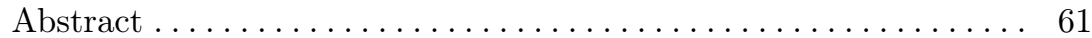

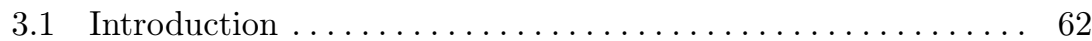

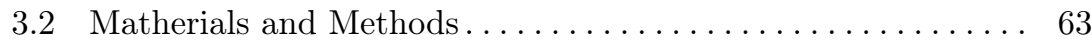

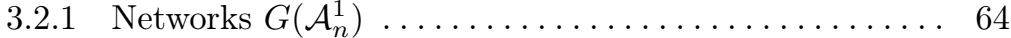

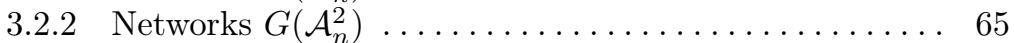

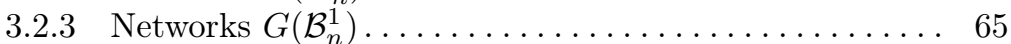

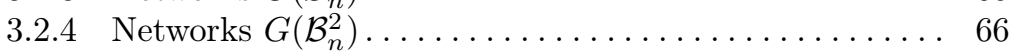

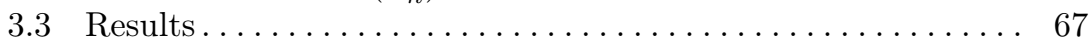

3.3 .1 Degree distribution . . . . . . . . . . . . . . . . . 67

3.3 .2 Density and Sparsity . . . . . . . . . . . . . . . . . . . 69

3.3.3 Local clustering coefficient . . . . . . . . . . . . . . . 70

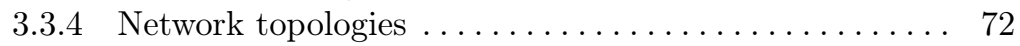

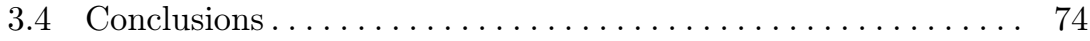

$4 \quad$ Discussion of the results and conclusions $\ldots \ldots \ldots \ldots \ldots 77$

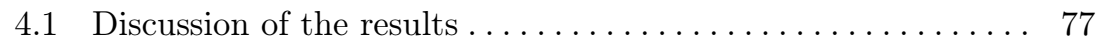

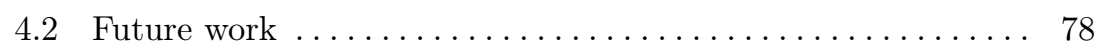

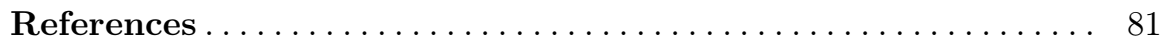




\section{Abstract}

The study of Complex Systems is one of the scientific fields that has had the highest productivity in recent decades and has not ceased to fascinate the community dedicated to studying its properties. In particular, Network Science has proven to be one of the most prolific areas within Complex Systems. In recent years, his methods have been applied to model multiple phenomena in real life, both naturally generated, such as in biology, and due to the actions and interactions of man, such as social networks or communication networks.

Recently, it has been seen how the methods of Network Science can be applied in the context of mathematics, as is the case of Number Theory. One of the most studied cases is networks whose elements are numbers and which are related through the divisibility relation. The main objective of this thesis is to extend these studies to other sets of numbers. On the one hand, we study the divisibility in natural numbers when we obtain these from Pascal matrices of increasing size, which allows us to extract non-sequential sets of numbers with non-constant increments between them. On the other hand, we study the case of the divisibility relation of rational numbers. Cantor's diagonal argument provides a way to order all rational numbers, which allows us to check to what extent some of the properties observed for the divisibility of natural numbers are extensible to a more general context.

The thesis is divided into 4 Chapters. Chapter 1 contains a general introduction to the thesis and it is structured into 6 sections. In Sections 1.1 and 1.2 , we briefly introduce Network Science, show some application examples, and motivate the study of networks of numbers generated from the divisibility property. In Section 1.3, we define the objectives of this $\mathrm{PhD}$ thesis and its scope. In Section 1.4, we present the notion of network, its representations, and some measures that can be calculated on them, such as nodes degrees, their distribution, the assortativity and the clustering coefficients.

In another hand, in Section 1.5, we review the best-known network models such as Erdös and Rényi random networks, Watts and Strogatz small-world networks, Barabási and Albert scale-free networks, and hierarchical networks. Finally, at the end of this Chapter 1, we show in Section 1.6 a review of various studies carried out in order to apply Network Science methods to problems and properties that arise in Number Theory, such as divisibility 
networks or networks generated from Collatz's Conjecture. or Goldbach's Strong Conjecture.

In Chapters 2 and 3, we show the results obtained and that have been published to date. Finally, in Chapter 4, we summarize the conclusions obtained and indicate some related problems that we consider of interest to address in the future. 


\section{Resumen}

El estudio de los Sistemas Complejos es uno de los campos científicos que ha tenido mayor productividad en las últimas décadas y no ha dejado de fascinar a la comunidad que se dedica al estudio de sus propiedades. En particular, la Ciencia de Redes se ha mostrado como una de las áreas más prolíficas dentro de los Sistemas Complejos. En los últimos años, sus métodos han sido aplicados para modelar múltiples fenómenos de la vida real tanto generados de manera natural, como puede ser en el caso de la biología, como debidos a las acciones e interacciones del hombre, como puede ser el caso de las redes sociales o las redes de comunicaciones.

Recientemente, se ha visto cómo los métodos de la Ciencia de Redes pueden ser aplicados en el contexto de las matemáticas, como es el caso de la Teoría de Números. Uno de los casos que más se han estudiado es el de las redes cuyos elementos son números y que se relacionan mediante la relación de la divisibilidad. El objetivo principal de esta tesis es extender estos estudios a otros conjuntos de números. Por una parte, estudiamos la divisibilidad en los números naturales cuando obtenemos estos a partir de subconjuntos de números naturales extraídos de matrices de Pascal de orden creciente, lo que nos permite extraer conjuntos de números de manera no secuencial y con incrementos no constantes entre ellos. Por otra parte, estudiamos el caso de la relación de divisibilidad de los números racionales, dado que a partir del argumento diagonal de Cantor se pueden ordenar, lo que nos permite comprobar hasta qué punto algunas de las propiedades observadas para la divisibilidad de los números naturales son extensibles a un contexto más general.

La tesis se divide en 4 capítulos. El capítulo 1 contiene una introducción general a la tesis y está estructurado en 6 secciones. En las secciones 1.1 y 1.2, presentamos brevemente la Ciencia de Redes, mostrando algunos ejemplos de aplicación y motivamos el estudio de redes de números generadas a partir de la propiedad de divisibilidad. En la Section 1.3, definimos los objetivos de esta tesis doctoral y su alcance. En la sección 1.4, presentamos la noción de red, sus formas de representación y algunas medidas que se pueden calcular sobre ellas, como son los grados de los nodos, la distribución de estos grados, la asortatividad y los coeficientes de clustering.

Por otro lado, en la Sección 1.5, revisamos los modelos de redes más conocidos como son las redes aleatorias de Erdös y Rényi, las redes de pequeño mundo de Watts y Strogatz, las redes libres de escala de Barabási y Albert y las redes jerárquicas. Mostramos en la Sección 1.6, una revisión de diversos estudios realizados con el fin de aplicar métodos de la Ciencia de Redes a problemas y propiedades que surgen en la Teoría de Números, como son las redes de divisibilidad o redes generadas a partir de la Conjetura de Collatz o la Conjetura Fuerte de Goldbach.

En los Capítulos 2 y 3, mostramos los resultados obtenidos y que han sido publicados hasta la fecha y, finalmente, en el Capítulo 4, resumimos 
las conclusiones obtenidas e indicamos algunos problemas relacionados que consideramos de interés abordar en un futuro. 


\section{Resum}

L'estudi dels Sistemes Complexos és un dels camps científiques que ha tingut major productivitat en les últimes dècades i no ha deixat de fascinar a la comunitat que es dedica a l'estudi de les seues propietats. En particular, la Ciència de Xarxes s'ha mostrat com una de les àrees més prolífica dins dels Sistemes Complexos. En els últims anys, els seus mètodes han sigut aplicats per a modelar múltiples fenòmens de la vida real tant generats de manera natural, com pot ser en el cas de la biologia, com deguts a les accions i interaccions de l'home, com pot ser el cas de les xarxes socials o les xarxes de comunicacions.

Recentment, s'ha vist com els mètodes de la Ciència de Xarxes poden ser aplicats en el context de les matemàtiques, com és el cas de la Teoria de Números. Un dels casos que més s'han estudiat és el de les xarxes els elements de les quals són números i que es relacionen mitjançant la relació de la divisibilitat. L'objectiu principal d'aquesta tesi és estendre aquests estudis a altres conjunts de números. D'una banda, estudiem la divisibilitat en els nombres naturals quan obtenim aquests a partir de matrius de Pascal de grandària creixent, la qual cosa ens permet extraure conjunts de números de manera no seqüencial i amb increments no constants entre ells. D'altra banda, estudiem el cas de la relació de divisibilitat dels nombres racionals, atés que a partir de l'argument diagonal de Cantor es poden ordenar, la qual cosa ens permet comprovar fins a quin punt algunes de les propietats observades per a la divisibilitat dels nombres naturals són extensibles a un context més general.

La tesi es troba dividida en 4 Capítols. El capítol 1, conté una introducció general a la tesi i està estructurat en 6 seccions. En les seccions 1.1 i 1.2, presentem breument la Ciència de Xarxes, mostrant alguns exemples d'aplicació i motivem l'estudi de xarxes de números generades a partir de la propietat de divisibilitat. En la Section 1.3, definim els objectius d'aquesta tesi doctoral y el seu abast. En la Secció 1.4, presentem la noció de xarxa, les seves formes de representació i algunes mesures que es poden calcular sobre elles, com són els graus dels nodes, la distribució d'aquests graus, la asortatividad i els coeficients de clustering.

En la Sección 1.5, revisem els models de xarxes més coneguts com són les xarxes aleatòries de Erdös i Renyi, les xarxes de xicotet món de Watts i Strogatz, les xarxes lliures d'escala de Barabási i Albert i les xarxes jeràrquiques. Mostrem en la Sección 1.6 una revisió de diversos estudis realitzats amb la finalitat d'aplicar mètodes de la Ciència de Xarxes a problemes i propietats que sorgeixen en la Teoria de Números, com són les xarxes de divisibilitat o xarxes generades a partir de la Conjectura de Collatz o la Conjectura Forta de Goldbach.

En els Capítols 2 i 3, vam mostrar els resultats obtinguts i que han sigut publicats fins hui i, finalment, en el Capítol 4, resumim les conclusions obtin- 
gudes i indiquem alguns problemes relacionats que considerem d'interés abordar en un futur. 


\section{Introduction}

Nowhere is the impact of network science more evident than in the scientific community.

Albert-László Barabási.

A network is a mathematical concept that is used to model systems where one can establish relationships (edges) between different components (nodes). When the network has non-trivial topological features, its study falls within the field of Network Science. In many systems of interest, one can identify a network that represents how its parts are interconnected $\left[\mathrm{B}^{+} 16\right.$, Introduction]. Network science is naturally contextualized as part of complex systems theory. Complex systems are those whose behavior is intrinsically difficult to model due to the interactions between their parts or with an environment. The multiple applications of Network Science have made it a very active area within the scientific community, particularly in complex systems.

As discussed in $\left[\mathrm{B}^{+} 16\right.$, Introduction], the most cited papers within complex systems are WS98 and BA99. These two papers are much more cited than those which are the most cited papers in other branches of complex systems, like Chaos Theory (Butterfly Effect Lor95]), neural networks [Hop82, renormalization group theory [Wil75], spin-glasses [EA75, or fractals [Man82]. As a matter of fact, the discoverer of scale-free networks, AlbertLásló Barabási is one of the most cited scientists in the world (at the early of September 2021, he was cited 269202 times, with h-index=159).

A fundamental starting discovery in network science was the Graph Theory, with the solution of the problem of the Königsberg Bridges by Euler. It further developed through the study of Random Networks models by Erdös and Rényi in [ER59, ER60. Importantly, by the turn of the century, complex topologies of networks were introduced, giving rise to what we understand nowadays as Network Science: The first one was the small-world network model by Watts and Strogatz in [WS98], the second one was the scale-free network model discovered by Barabási and Albert [BA99].

Random networks were first introduced as a mathematical topic, with no real impact on real-world problems, as the connections in physical or social systems are not random. The properties of these networks were far from real, 
observed networks $\mathrm{B}^{+} 16$, Ch. 3.10], [New18]. The introduction of the smallworld and scale-free hallmarked a research effort that approached the theory to real-world phenomena.

\subsection{Examples of application of complex networks}

In the previous section, we discussed the impact of the discovery and introduction of the small-world and scale-free concepts, highlighting their importance in describing the complexities of real-world phenomena. This effort has continued up to today, where directed networks, networks of networks, spatial modular networks, etc., are used to get insight into various applications, such as:

1. The study of the effects of natural phenomena such as global warming, extreme weather events, and earthquakes can be performed from a network perspective. So far, physics has advanced to understand the structure and pattern of climatic phenomena using network approaches. However, the network science approach applied to these types of phenomena could offer alternatives to predict the complexity of phenomena such as earthquakes, the strength of El Niño events, increased air pollution, etc. $\mathrm{FMC}^{+} 16, \mathrm{GGB}^{+} 12, \mathrm{BBB}^{+} 14, \mathrm{MBMK} 12$.

2. A complex network science approach to study climate phenomena, which has allowed the generation of a type of complex network called climate networks. Here, the nodes stand for the geographic locations where the natural phenomenon occurs. The level of similarity, also called correlation, between the records (temperature, pressure, winds, precipitation, increased rainfall in some regions, and droughts in other regions, decreased fishing, famine, or plagues) represent the links. [ $\left.\mathrm{FMC}^{+} 16\right]$.

3. The interrelationship between physiological systems and the underlying properties of complex networks has permitted us to develop a framework to probe the interactions between various systems. This has allowed constructing a comprehensive physiological network, which undergoes dynamic transition phases that are associated with a rapid reorganization of physiological interactions on time scales of about a few minutes, indicating high network flexibility in response to disturbances $\left[\mathrm{BBK}^{+} 12\right]$.

4. Applications of complex network properties related to the optimization of electrical power systems, allowing the study and characterization of a greater resistance of the network, a reduction in energy losses and line load, better voltage stability, and a supply to critical loads during a blackout [SEM18].

5. The application of complex network concepts that permit to explain the stability of large and complex ecosystems has shown that the trophic coherence determines food stability [JDGDM14. Here the authors construct a network in which the nodes are the species, and the predator-prey interactions determine the edges. 
6. The development of an optimal strategy that allows immunizing a population with the minimum possible dose $\left[\mathrm{CPH}^{+} 08\right.$, or a population whose members are connected following a scale-free network pattern. Although this last strategy does not require knowledge of the nodes or the degrees of connectivity of each subject with respect to the others. It is highlighted that these populations follow a distribution of broad degrees, which implies the existence of hubs and, therefore, it is of vital importance the treatment of centrality, as an inherent property of networks that follow a power-law CHBA03.

7. In the same direction, the efficient immunization of a network was proposed by extracting an amount $n$ of its nodes, immunizing the node with the greatest centrality. Then this process is repeated sequentially, allowing what the authors call "targeted immunization". On the other hand, they determine the critical percolation threshold $p_{c}$ and the size of the giant component $P_{\infty}$. Since the number of $n$ nodes that can be taken varies, i.e., $1 \leq n \leq \infty$, the authors have found a new general scale relationship between $\left|p_{c}(\infty)-p_{c}(n)\right|$ and in particular the relation for $n$ as, $\left|p_{c}(\infty)-p_{c}(n)\right| \sim n^{-1} \exp (-\alpha n)$. Concerning scale-free networks, they have determined that $p_{c}$ has a transition from zero to nonzero values, as $1 \leq n \leq \mathcal{O}(\log N)$, where $N$ is the size of the network [LSD $\left.{ }^{+} 20\right]$.

8. Applications of complex modular structural networks to simulate and estimate the probability of the spread of diseases worldwide, such as pandemics, applying properties related to hierarchical networks [VBH20.

9. The application of the measures of centrality and nested communities has permitted in the field of psychiatry has allowed explaining the underlying relationships (causal relationships) between the different diseases or pathologies that the same patient has (comorbidity), to make better diagnoses [HC18].

10. The study and characterization of urban and highway transport flows through applying the property of complex networks called "percolation," which is understood as the filtration transition process in a dynamic traffic network that is seen affected by bottlenecks, which evolves as time passes. In this sense, the authors have proposed a new way of organizing traffic, simulating it as a network that follows a percolation process. The underlying idea is to show that global traffic is broken when the identified bottlenecks are congested $\left[\mathrm{LFW}^{+} 15\right]$. In this direction and using complex networks again, it has been possible to identify multiple abrupt phase transitions in urban transport congestion [LBHGSR21].

\subsection{A perspective on divisibility networks}

One active area of research within Network Science is that the network itself is built according to a mathematical recipe or numeric relation. The nodes 
inherit the connections among them (edges) and the strength of these interactions from some mathematical structure. With this idea, we can construct complex artificial networks in which their properties are related to the underlying mathematical structure. This allows to both give new insight on this mathematical structure and to find if this path leads to interesting networks with application in some real-world problems, see Section 1.6. This thesis is devoted to the study of these types of networks.

The first works in this area appeared circa 2005. One of the foundational works was realized by Chandra et. al., who studied the Goldbach Strong Conjecture (GSC) [SL84] using concepts from complex networks theory CD05. This work allowed to find a probability function $P$ that establishes the minimum distance between pairs of possible primes that sum up to a certain even number; that is, $P=|p-q|^{\alpha}$, with $(-\infty<\alpha<\infty)$, where $p$ and $q$ are prime numbers such that $n_{e}=p+q$ is an even number. We recall that according to Goldbach's conjecture, any even number can be broken up as the sum of two prime numbers. The authors report that if $\alpha>-1.8$, then the network constructed from this relation presents a small-world topology, i.e., the average shortest distance is small (increases only logarithmically with the size of the network). However, the clustering coefficient, which measures the tendency of the nodes in a graph to cluster together, is high compared to the random network. Conversely, if $\alpha<-1.8$, the network is regular [WS98].

Zhou et. al., were the first to analyze divisibility networks of natural numbers [ZWHC06]. The originality of this idea was to establish edges between the nodes (which are the natural numbers) through divisibility relations in modulo zero between these nodes. The result was a network that presents scale-free properties; that is, the probability for a node to have $k$ connections is $p_{k} \approx k^{-2}$. The clustering coefficient is relatively high at the rate of $C \approx 0.34$. Also, the average path length in whatever place of the network, which is the average number of steps along the shortest paths for all possible pairs of network nodes, is upper bounded independently of the network size. In their work, authors considered arbitrary networks of natural numbers, i.e, $G=(N, E)$ (here $N$ is the set of nodes, which are natural numbers, i.e. $N \in \mathbb{N}>1$, and $E$ the collection of edges). The edges follow the following rule: for all $a, b \in N$ there is an edge between them if and only if $a \mid b$ or $b \mid a$ in modulo zero. However, it turns out that in the limit, there will always be some prime number $p \in N$ that is not divisible in modulo zero with some value ( $a$ or $b$ ), and therefore the distance between $p$ and $a$ or $p$ and $b$ is infinite.

To illustrate this let us suppose that $p$ is a prime such that $p \in V$, where $V$ is the set of all prime numbers. On the other hand, if we denote as $q=\min (V)$ such that $q>1$, then, in the limit, there will always be some value $\Omega=p \mid q$, such that, $\Omega \notin \mathbb{Z}^{+}$. This means that the degree (number of connections) of $p$ is zero, that is, $k_{p}=0$, and as we will see in Section 1.5.3.1 if $k=0$, then 
the constant $C$ of the equation $p_{k}=C \cdot k^{-\gamma}$ does not converge, and then, the path length between nodes $p$ and $q$ goes to infinity.

This apparent problem was solved by Shekatkar et. al., in [SBA15] where they built undirected networks, similar to those of Zhou et. al., in which the nodes are the natural numbers and the divisibility relation between them gave the edges. Among the most important results provided, they found analytical expressions for the calculation of local clustering coefficient, $C_{n}$ where $n$ is the number of different factors of a given node, that is, its number of connections (see [SBA15, Eq. 2]), in terms of the network size. They concluded that the value of $C_{n}$ depends only on the number of different prime factors of $n$ and their powers, without considering the prime values present there. This result allowed them to conclude that $C_{n}$ remains constant for values $\frac{N}{2}<n<N$, with $N$ the total size of the network. This led them to discover a new property called similary stretching, (see [SBA15, Figs. 4 \& 5]).

In this Ph.D. thesis, we take on the ideas from Shekatkar et. al., extending them to some subsets of natural numbers extracted from the Pascal triangle and extending them to the set of rational numbers in the unit interval, too. In this sense, we have defined the following general and specific objectives.

\subsection{Objectives}

The general objective of this doctoral thesis is to study the topology of complex networks in which the nodes are real numbers and connectivity is established through divisibility relations in zero modulus, between them. To achieve this general objective, we have established the following specific objectives:

1. Study the state of the art related to the construction of complex networks whose nodes are real numbers.

2. Determine the numerical sets that had not yet been previously studied, from the perspective of complex network science.

3. Characterize the connectivity relationships of the previously identified numerical sets.

4. Establish the Kolmogorov-Smirnov goodness fit test to determine if the behavior of the data distribution is similar to the power-law distribution.

5. Program the necessary algorithms that allow us to obtain the adjacency matrices, to later study the properties of the complex networks identified. 


\title{
1.4 Preliminaries
}

\author{
Few research fields can trace their birth \\ to a single moment and place in history. \\ Graph theory, the mathematical scaffold \\ behind network science, can.
}

Albert-László Barabási.

This section introduces the theoretical foundations necessary for the analysis and study of complex networks. These concepts will be used in the following sections, and we collect them here for an easy reference. Further insights in network theory can be found in, e.g., [ $\mathrm{B}^{+} 16$, New18, Est12, AAH02, Wea96].

\subsubsection{What is a network?}

First, we introduce the concept of a complex network which, in few words, is just a graph. We define a complex network of the form $G=(V, E)$ as a mathematical structure consisting of a collection of nodes joined by edges. Complex network Theory and Graph Theory are nevertheless commonly understood as slightly different topics, due to the approach to study these objects: In Graph Theory one is more concerned with heuristics and algorithms applied to these elements; while in Complex Network Science one is more concerned with studying the topology derived from the connections between the nodes.

Therefore, we can look at the set of nodes, or vertices $V$, as independent elements or phenomena, whose relations among them are given by the set of edges or links $E$. These edges usually represent one or more characteristics shared by pairs of nodes. With the incorporation of computers and the increase of their computational power, some datasets started to be analyzed: The first studied examples were the actor collaboration network, the World Wide Web, the electrical power grid network in the US [BA99], or communication networks Arm98, and the spreading of viruses on them [PSV01]. See Table 1.4.1 for further details about some of the most studied examples of networks.

\subsubsection{Basic notation and network measures}

In this section, we introduce the main notation and measures used in network science. Unless otherwise stated, we will refer to an arbitrary network $G=$ $(V, E)$, where $V$ is a non-empty set of nodes, denoted by $v_{1}, \ldots, v_{n}$, and $E$ is the set of edges given by a set of pairs of elements of $V$. If the order of the elements in these pairs is relevant, we will say that the network is directed; if not, we will say that it is undirected. 


\begin{tabular}{lll}
\hline Network & Node $(V)$ & Edge $(E)$ \\
\hline Internet & routers & internet connections \\
World Wide Web & web pages & hyperlinks \\
Power grid & power plants & cables \\
Phone calls & phone numbers calls \\
Email & email addresses emails \\
Science collaboration scientists & co-authorship \\
Actor network & Actors & Coappearance movies \\
Citation network & Papers & Citations \\
Protein interactions & proteins & binding interactions \\
Food web & species & predation \\
Neural networks & neurons & synapses \\
Metabolic networks & metabolic & metabolic reactions \\
Friendship networks & people & friendship relations \\
\hline
\end{tabular}

Table 1.1: Examples of nodes and edges from different networks $\mathrm{B}^{+} 16, \mathrm{Ch}$. 2.3] and [New18, Ch. 6.1].

The networks studied in this thesis will be undirected, so we will introduce the following definitions and measures just for this type of networks. Besides, our networks will be simple, without loops connecting a node with itself by a single edge.

\subsubsection{Adjacency matrix}

There are two fundamental mathematical concepts to represent a network: the Laplacian matrix and the adjacency matrix. However, the latter is the most widely used concept to study the connectivity relationships between the nodes of a network. This matrix provides the complete description of the links between nodes. The adjacency matrix $A_{n}$ of a network $G=(V, E)$ is given by a square $n \times n$ matrix whose elements $A_{i j}$ are set as follows:

$$
A_{i j}= \begin{cases}1, & \text { if there is an edge between the nodes } i \text { and } j \\ 0, & \text { otherwise. }\end{cases}
$$

To illustrate the construction of an adjacency matrix, we consider the network in Fig. 1.1, which has 6 nodes and 7 edges.

From Fig. 1.1, we can obtain its adjacency matrix,

$$
A=\left(\begin{array}{llllll}
0 & 0 & 0 & 0 & 1 & 1 \\
0 & 0 & 1 & 0 & 0 & 0 \\
0 & 1 & 0 & 1 & 1 & 1 \\
0 & 0 & 1 & 0 & 0 & 1 \\
1 & 0 & 1 & 0 & 0 & 0 \\
1 & 0 & 1 & 1 & 0 & 0
\end{array}\right) .
$$




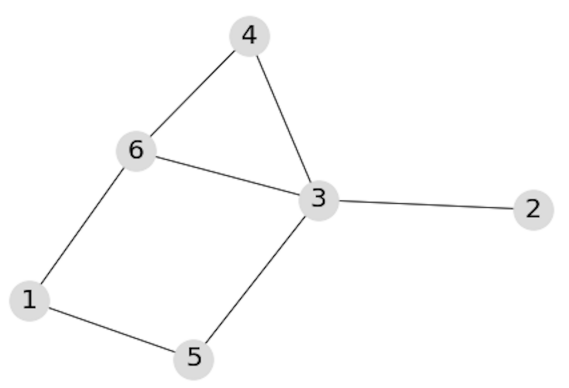

Fig. 1.1: Network notation. This network has 6 nodes and 7 edges.

We can see that the matrix is symmetric with respect to its main diagonal, which means that we are considering an undirected network. It is worth mentioning that, in general, these matrices need not be symmetric. If this was the case, we say that we have a directed network. In our graphs, we will consider simple networks without loops connecting a node with itself.

\subsubsection{Degrees in networks}

Given an undirected network $G=(V, E)$, we call the degree of the node $v_{i}$ as the number of edges connected to it, and we denote it by $k_{i}$. Then, the total number of edges $|E|$ will be given by $E=\frac{1}{2} \sum_{i=1}^{N} k_{i}$. The degree of the node $v_{i}$ can also be obtained from the adjacency matrix as $k_{i}=\sum_{j=1}^{N} A_{i j}$. Then, the total number of edges can also be estimated as $|E|=\frac{1}{2} \sum_{i=1}^{N} k_{i}=$ $\sum_{1 \leq i, j \leq N} A_{i j}$.

One of the most important properties of a network is its average degree. This measure gives us an idea of the average stability of the connections between nodes by measuring the number of edges compared to the number of nodes. We call the average degree $\langle k\rangle$ to the average number of connections of all the nodes in a network, given by

$$
\langle k\rangle=\frac{1}{N} \sum_{i=1}^{N} k_{i}=\frac{2|E|}{|V|} .
$$

In many large networks, we have that $\langle k\rangle \ll N \ll|E|$. In our small example, in Fig. 1.1. its average degree is $\langle k\rangle=\frac{2 \times 7}{6} \approx 2.33$. This means that each node has, on average, approximately 2.33 edges.

We can also estimate the percentage of existing edges with respect to the maximum possible number of edges. In a graph with $N$ nodes this will be $\left(\begin{array}{c}N \\ 2\end{array}\right)=\frac{1}{2} N(N-1)$. Therefore, we can define the density of a network $G=(V, E)$, denoted by $\rho(G)$, as the fraction of existing edges respect to the maximum number of admissible edges 


$$
\rho(G)=\frac{E}{\left(\begin{array}{c}
N \\
2
\end{array}\right)}=\frac{2 E}{N(N-1)}=\frac{\langle k\rangle}{N-1} .
$$

Since most undirected networks have a large number of nodes, then the estimation of $\rho(G)$ can be reduced to

$$
\rho(G)=\frac{\langle k\rangle}{N} .
$$

This last expression shows that the density gives us information about the probability of a pair of nodes being connected at random by an edge, therefore $\rho(G) \in[0,1]$. In our example, we have $\rho(G) \approx 0.39$.

Given a network $G=(V, E)$, we say that $H=(W, F)$ is a subnetwork of $G$ if $\emptyset \neq W \subseteq V$ and $F \subseteq E$. Let us consider a family of subnetworks $H_{n}=\left(W_{n}, F_{n}\right)$ of $G$ with $\left|W_{n}\right|=n$ and $W=\cup_{n} W_{n}, F=\cup_{n} F_{n}$ and $H=\cup_{n} H_{n}$. If $\rho\left(H_{n}\right)$ tends to 0 as $n$ tends to $|V|$, then we say that the network is sparse. Otherwise, we say that the network is dense.

\subsubsection{Degree distributions}

Throughout this section, we describe one of the most fundamental properties of networks, their degree distribution $\{p(k)\}_{k=0}^{\infty}$. The network can be studied through the degree distribution $p(k)$, which accounts for the frequency with which nodes of degree $k$ appear in a $G$. That is, one has to count how many nodes have each degree $k$, which we denote as $N(k)$, and divide by the total number of nodes in the network in order to obtain the fraction of nodes in the network with degree $k$, i.e.

$$
p(k)=\frac{N(k)}{|V|} .
$$

This provides the probability distribution that a randomly selected node in the network has degree $k$. Since it is a probability distribution, it fulfills that

$$
\sum_{k=0}^{\infty} p(k)=1 .
$$

In Fig. 1.2, we represent the degree distribution of the network example considered in this section. We have $p_{1}=1 / 6$, since one of the six nodes has degree $k_{2}=1$. In the same way, we get $p_{2}=1 / 2$ (three of the six nodes have degree $\left.2, k_{1}=k_{4}=k_{5}=2\right), p_{3}=1 / 6$ (since $k_{6}=3$ ), and $p_{4}=1 / 6$ since $k_{3}=4$, and then, for every $k_{i} \geq 5$ we have null probabilities $p\left(k_{i}\right)=0$. We can easily check that $\sum_{k=0}^{\infty} p(k)=1$, as indicated in Eq. (1.7).

The degree distribution $\{p(k)\}_{k=0}^{\infty}$ has an average which evidently coincides with the average degree 


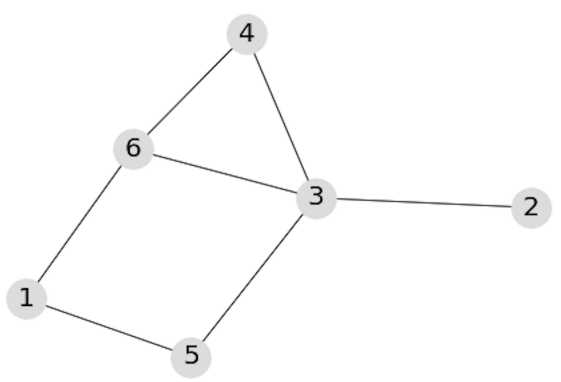

(a)

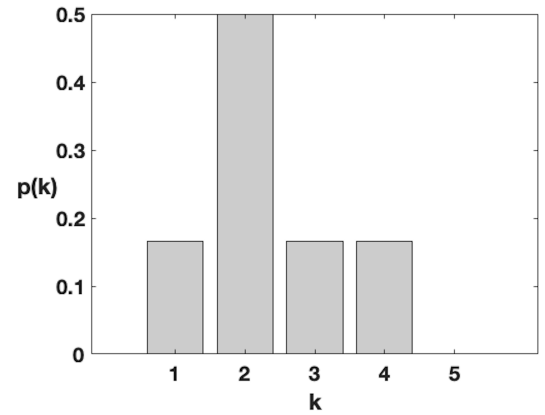

(b)

Fig. 1.2: Network representation and its degree distribution. We show our network example in Fig. 1.2a and its degree distribution in Fig. 1.2b.

$$
\langle k\rangle=\sum_{k=0}^{\infty} k \cdot p(k) .
$$

The degree distribution provides much information about the phenomena studied from the perspective of network theory, being a central part of studying the structure of a given network. Later, we will use the degree distribution to obtain different measures from it, particularly when it resembles a power-law (or scale-free) distribution (also known as Zipf's law or the Pareto distribution). In such case, the distribution is of the form $p(k) \propto k^{-\gamma}$, with $\gamma$ some constant typically falling in the interval $[2,3]$; thus, it has a well-defined average. Note that it has finite variance only if $\gamma>3$ and that there are also examples of power laws where $\gamma<2$, e.g., in continuous time random walks [New05.

Nodes in a network can be connected by a path that results in the concatenation of several edges. A network can be split into one or more subsets, with all the nodes in each subset being accessible from the rest and not accessible from any other node of any other subset. Each one of these sets is called a connected component. We say that a network is connected provided there is an unique connected component. We seldom have a connected network in real applications, but we have a huge connected component containing most nodes. Such a component is called the giant connected component.

Connectedness is closely related to the robustness of the network. A network is considered to be robust if it still contains a giant connected component after removing some of its nodes. In random networks, we see that this giant network can emerge at some phase transition. We will also see that scale-free networks usually contain one giant connected component. The connectivity property is strongly related to other fundamental properties of the network, 
such as the centrality, the existence of hubs, the degree distribution, the transitivity, the global and local clustering coefficients, the average degree, etc.

We can estimate the averaged distance between any pair of nodes or average path length, denoted by $\langle d\rangle$. It is the averaged distance between all pairs of nodes in the same connected component. This measure has sense if we consider that each edge has an associated distance. If this is not explicitly mentioned, we can say that all edges have a weight equal to 1 . It can be computed as

$$
\langle d\rangle=\frac{1}{N(N-1)} \sum_{1 \leq i, j \leq N} d_{i, j},
$$

where $d_{i, j}$ represents the distance between nodes $v_{i}$ and $v_{j}$, that is given by the shortest path connecting them. Clearly, if two nodes cannot be connected by a sequence of edges, then the distance between them will be $\infty$. So this measure is only relevant within the maximal subnetworks of a given one, with all their nodes connected among them by some of these paths.

Finally, we also recall that the maximum distance between any pair of nodes in a network is called the diameter of the network, also denoted as $d_{\text {max }}$. For a network with several connected components, we say that the diameter is $\infty$, since we can easily find a pair of nodes at $\infty$ distance.

\subsubsection{Assortativity}

We call the assortativity coefficient of a network $G=(V, E)$, denoted by $r(G)$ or simply by $r$, to the tendency of the nodes to connect to others with the same or similar characteristics. It is expressed in terms of the degrees of adjacent nodes and their connection preference in the whole network,

$$
r=\frac{\sum_{1 \leq i j \leq N}\left(A_{i j}-k_{i} k_{j} / 2|E|\right) k_{i} k_{j}}{\sum_{1 \leq i, j \leq N}\left(k_{i} \delta_{i j}-k_{i} k_{j} / 2|E|\right) k_{i} k_{j}},
$$

where $\delta_{i j}$ is the Kronecker delta, $k_{i}$ is the degree of the node $v_{i}$, and $A_{i j}$ stands for the corresponding element in the adjacency matrix of the network. It is worth mentioning that computing assortativity from Eq. 1.10 supposes a high computational cost. Therefore, we can approximate the assortativity using the expression New18

$$
r=\frac{S_{1} S_{e}-S^{2}}{S_{1} S_{3}-S_{2}^{2}}
$$

where

$$
S_{e}=\sum_{i j} A_{i j} k_{i} k_{j}=2 \sum_{l_{(i, j)}} k_{i} k_{j}, \quad \text { and } \quad S_{m}=\sum_{i=1}^{N_{n}} k_{i}^{m}
$$


for $m=1,2,3$. This coefficient ranges from -1 to 1 . If $r>0$, then we say that the network is assortative. If $r<0$, then we say that the network is disassortative, and if $r=0$, we say that it is non-assortative. Most empirical networks, except social networks, are in general disassortative or degree-degree anticorrelated. Johnson, et. al., found that this is in general due to the principle of maximum entropy [JTMM10.

\subsubsection{Clustering Coefficients}

The local clustering coefficient of a given node $i$ measures the proportion of its adjacent nodes which are connected among themselves. We can calculate the local clustering coefficient as

$$
C_{i}=\frac{2 E_{i}}{k_{i}\left(k_{i}-1\right)},
$$

where $E_{i}$ represents the number of links between the $k_{i}$ neighbors of node $i$. This value ranges from 0 to 1 , and it can be understood as the probability of interconnection between adjacent nodes of a given one. For instance, $C_{i}=0.5$ implies that there is a $50 \%$ chance that two adjacent nodes of a given one were connected between them. To illustrate this concept, let us consider the undirected network of Fig. 1.3a.

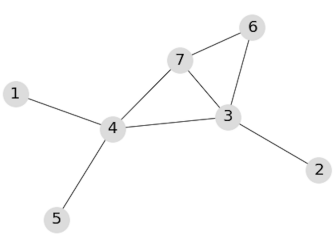

(a)

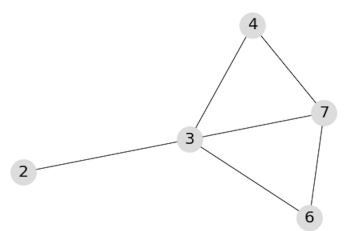

(b)

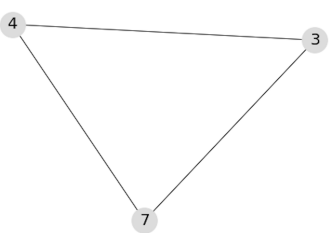

(c)

Fig. 1.3: Network representations: We have a network with 7 nodes and 8 edges in Fig. 1.3a. We represent the subnetwork of the adjacent nodes to node $v_{3}=3$ in Fig. 1.3b. We show the triangle formed by the vertices $v_{3}, v_{6}$, and $v_{7}$ in Fig. $1.3 \mathrm{c}$

Let us compute the local clustering coefficient of $v_{3}$, named $C_{3}$. First, we take the subnetwork determined by their adjacent nodes, see Fig. $1.3 \mathrm{~b}$ and we compute the number of edges between them, see Fig. $1.3 \mathrm{~b}$. Since $v_{2}$ is not connected to any other node, then $E_{2}=0$. Nodes $v_{4}$ and $v_{6}$ are only connected with $v_{7}$, so $E_{4}=E_{6}=1$. Since $v_{7}$ is connected with nodes $v_{4}$ and $v_{6}$, we have $E_{7}=2$. Therefore, $C_{3}=\frac{2(0+1+1)}{4(4-1)}=\frac{1}{3}$. The other clustering coefficients of this network are $C_{1}=0, C_{2}=0, C_{4}=1 / 6, C_{5}=0, C_{6}=1$, and $C_{7}=2 / 3$. 
Once computed the local clustering of each node, we can estimate the average local clustering coefficient of the whole network. It is denoted by $\langle C\rangle$ and it is obtained by calculating the mean between all the values of $C_{i}$, with $i=1, \cdots, N$, that is

$$
\langle C\rangle=\frac{1}{N} \sum_{i=1}^{N} C_{i}
$$

This coefficient lies between 0 and 1 , and it estimates the probability that two adjacent nodes to a randomly chosen node were connected by an edge. For our example in Fig. (1.3a), we have

$$
\langle C\rangle=\frac{(0+0+1 / 3+1 / 6+0+1+2 / 3)}{7}=\approx 0.31 \text {. }
$$

Another clustering measure is the global clustering coefficient, denoted as $C_{\Delta}$. We say that a triplet is any set of three nodes. The global clustering coefficient measures the total number of closed triplets in a network. It can be easily seen that a triangle $A B C$, like the one in Fig. 1.3c, has 3 triplets associated to it: $A B C, B C A$, and $C A B$. We define the global clustering coefficient as

$$
C_{\Delta}=\frac{3 \times \text { Number of triangles }}{\text { Number of connected triples }} \text {. }
$$

For a more accessible computational estimation of the global clustering coefficient, we can define it in terms of the elements of the adjacency matrix of the network

$$
C_{\Delta}=\frac{\sum_{i, j, k} A_{i j} A_{j k} A_{k i}}{\sum_{i} k_{i}\left(k_{i}-1\right)},
$$

where $A_{i j} A_{j k} A_{k i}=1$ represents a closed triplet. When the denominator is 0 , we say that $C_{\Delta}=0$.

If we return to Eq. 11.13, we can see that $E_{j}$ stands for the number of closed triplets that contain $v_{j}$, as each link between two adjacent nodes to a given one closes a triplet. 


\subsection{Network Models}

Network science aims to build models that reproduce
the properties of the real networks.

Albert-László Barabási.

The study of real-life phenomena modeled with networks has become a vey appealing field of study in the last decades. Three are the cornerstones that set the basis of Network Science: The random networks introduced by Erdös and Rényi (1959) [ER59] and Gilbert (1959) Gil59], the small-world networks (1998) introduced by Watts and Strogatz [WS98, Wat04, and the scale-free networks introduced by Barabási and Albert (1999) [BA99].

However, the modeling of real-life problems dates back to earlier times. The first to model this type of problem was Euler, in 1736, giving an ingenious and simple solution to the famous problems of the seven bridges of Königsberg. The solution to this problem gave rise as well know as Graph Theory. According to Barabási $\mathrm{B}^{+} 16$, Ch. 2.1], the Euler's ingenious resolution gives us two important messages: The first is that some problems become simpler and more tractable if they are represented as a graph. The second is that the existence of the path does not depend on our ingenuity to find it. Rather, it is a property of the graph. Indeed, given the structure of the Königsberg graph, no matter how smart we are, we will never find the desired path. In other words, networks have properties encoded in their structure that limit or enhance their behavior.

Unlike Graph Theory, Network Science studies the structure of the connections between the nodes based on the topological study of the network, which is essential to understand the interaction between the components (nodes). In this section, we review and analyze some of the most well-known topologies that arise from the connectivity relationships (edges) between the components. We will focus on the description of random networks, small-world networks, and scale-free networks.

\subsubsection{Random Networks}

Let us consider a set of nodes siting in specific places in space. We want to establish, randomly, routes that connect pairs of nodes. Suppose that node $v_{i}$ can be connected with the node $v_{j}$ with certain probability. This type of phenomenon occurs when we are in the presence of random networks. These models were firstly introduced by Erdös and Rényi in 1959 [ER59, ER60], and they are also known as Erdös-Rényi networks.

According to Newman, et. al., New18 a random network is a specific type of network in which we fix a predetermined set of parameters, usually the 


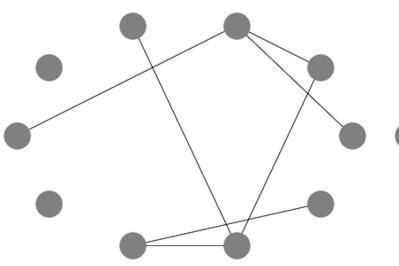

(a)

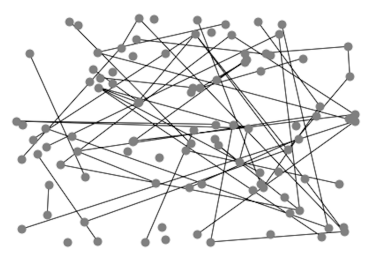

(d)

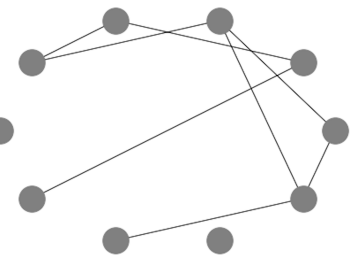

(b)

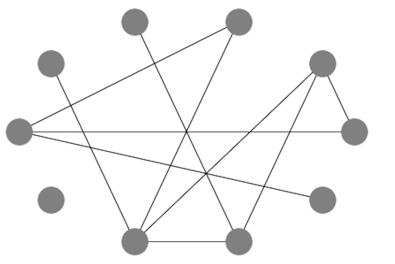

(c)

Fig. 1.4: Representation of two random network models $G(N, p)$. Panels (a), (b) and (c) represent three instances of $G(10,0.15)$. We observe that the number of edges is different for each simulation, that is, $(L=7,8,9)$. Panels (d), (e) and (f) show three instances of $G(100,0.01)$. Here there are a lot of nodes with degree $k=0$.

number of nodes $N=|V|$, the number of edges $L=|E|$, and the probability $p$, in such a way that the edges are connected to the nodes in a random way. To build a random network we carry out one of the following approaches.

Random networks with $N$ nodes, where the edges are generated following a binomial probability distribution of parameter $p$. These networks are denoted by $G(N, p)$.

1. We consider a fixed number of nodes $N$ and a probability $p \in[0,1]$.

2. We choose a pair of nodes $v_{i}, v_{j} \in V$ and a random number $p_{0}$ between 0 and 1 . Then we connect the pair of nodes $v_{i}, v_{j}$ if $p_{0}<p$ and we leave them disconnected if $p_{0} \geq p$.

3. Step 2 must be repeated for each one of the $N(N-1) / 2$ different pairs of nodes.

Random networks with $N$ nodes and $L$ edges where the nodes connect to each other, randomly, using one of those $L$ edges. We denote them by $G(N, L)$. They are generated as follows:

1. We consider a fixed number of nodes $N$ and edges $L$.

2. We choose randomly a pair of disconnected nodes $v_{i}, v_{j} \in V$ and we connect them by an edge. 


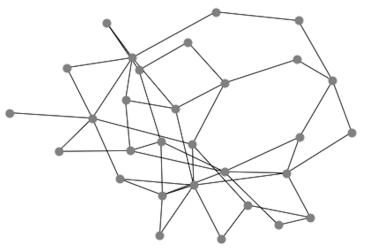

(a)

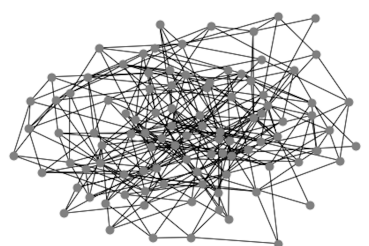

(d)

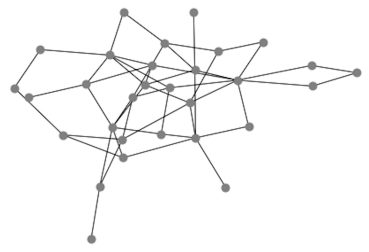

(b)

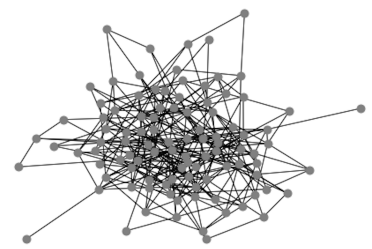

(e)

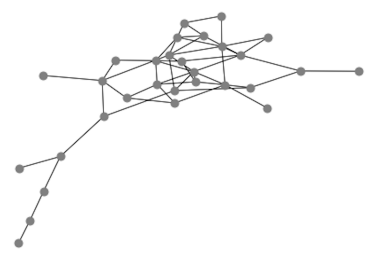

(c)

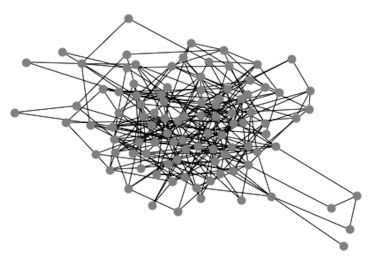

(f)

Fig. 1.5: Representation of two random network models $G(N, L)$. Panels (a), (b) and (c) represent three instances of $G(30,50)$. We observe that the number of edges is 50 in the three cases. Panels (d), (e) and (f) show three instances of $G(100,300)$. Each one contains 300 edges.

3. Step 2 must be repeated for each one of the $L$ edges.

We illustrate examples of these networks in Figs. 1.4 and 1.5.

\subsubsection{Degree distribution}

It is challenging to compute properties and topologies of networks generated under the Erdös-Rényi model $G(N, L)$ because of the underlying nature of the dynamics of the edges allocation. Of course, some properties that depend on the number of links, such as the average degree $\langle k\rangle=2 L / N$ are easy to compute.

However, we do not know the probability of a pair of nodes $v_{i}, v_{j}$ to be connected. Then, it is very hard to compute the degree distribution $\{p(k)\}_{k=0}^{\infty}$, the local clustering coefficient $C_{i}$, the average path length $\langle d\rangle$, etc. This is why most of the work and algorithm development have been focused on the random networks of type $G(N, p)$. Further details on the following estimations

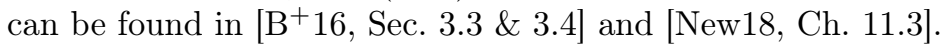

Given a fixed number of nodes $N$ and a probability $p$ one can have slightly different network configurations since the number of edges may vary. The probability that a random network has exactly $L$ links depends on the next terms: The probability that the node $v$ was connected to $L$ specific nodes is 
$p^{L}$, but we have to multiply it by the probability that it will not be connected with the rest, that is $(1-p)^{N(N-1) / 2-L}$. There are $\left(\begin{array}{c}N(N-1) / 2 \\ L\end{array}\right)$ possibilities to plug the $L$ edges with the $N$ nodes. Therefore, the probability that the network generated under the parameters above has $L$ edges is equal to

$$
p_{L}=\left(\begin{array}{c}
N(N-1) / 2 \\
L
\end{array}\right) p^{L}(1-p)^{N(N-1) / 2-L} .
$$

This is a binomial distribution. The expected number of edges of a network of type $G(N, p)$ will be given by

$$
\langle L\rangle=\sum_{L=0}^{\frac{N(N-1)}{2}} L p_{L}=p \frac{N(N-1)}{2} .
$$

Hence, this is equal to the probability $p$ multiplied by the number of attempts to connect an edge. Then, we can easily obtain the average degree of the network $\langle k\rangle$, which reads

$$
\langle k\rangle=\frac{2\langle L\rangle}{N}=p(N-1) .
$$

Let us show how to obtain the degree distribution $\{p(k)\}_{k=0}^{\infty}$. If we revise again Figs. 1.4 and 1.5. we can observe that each realization has a different number of edges. Moreover, we have some nodes that have more connections than others. The degree distribution should capture these differences. Let us compute it for random networks of type $G(N, p)$.

Let us consider an arbitrary node $v$ of a random network of type $G(N, p)$. This node may be connected with each one of the other $(N-1)$ nodes with probability $p$. This means that the probability of being connected, in particular, to $k$ nodes and not to the rest is given by: $p^{k}(1-p)^{N-1-k}$. Now, we can choose $k$ edges of the $N-1$ potential edges that a node can have, which gives $\left(\begin{array}{c}N-1 \\ k\end{array}\right)$ different combinations. Therefore, the total probability that a node was connected exactly with $k$ of the other $N-1$ nodes is

$$
p(k)=\left(\begin{array}{c}
N-1 \\
k
\end{array}\right) p^{k}(1-p)^{N-1-k},
$$

which is a binomial distribution of parameters $N-1$ and $p$ that represent the network size and probability of connection. The standard deviation for this distribution will be

$$
\sigma=[p(1-p)(N-1)]^{1 / 2} .
$$

Nevertheless, when $\langle k\rangle \ll N$, it is common to approximate the binomial by a Poisson distribution because it provides an analytic expression that is easier to manipulate in subsequent calculations. The condition $\langle k\rangle \ll N$ is usually satisfied in real networks since most of them are sparse. When $p$ 
is small, the degree distribution of random networks $G(N, p)$ can be well approximated by a Poisson distribution, see Fig. 1.6a.

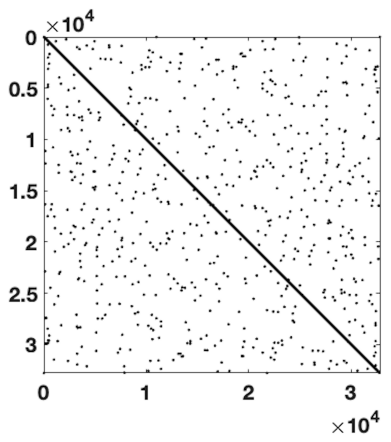

(a)

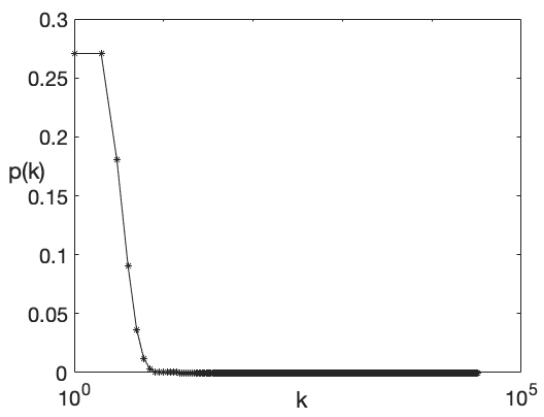

(b)

Fig. 1.6: Representation of the sparse adjacency matrix of a network $G\left(2^{15}, 0.01\right)$ (left) and its degree distribition (right).

For a network with average degree $\langle k\rangle$, its degree distribution $\{p(k)\}_{k=0}^{\infty}$ can be approximated by the following Poisson distribution

$$
p(k)=e^{-\langle k\rangle} \frac{\langle k\rangle^{k}}{k} .
$$

We can easily check that the binomial and Poisson distributions have a peak around $\langle k\rangle$, as expected. Besides, the distribution width is also controlled by $\langle k\rangle$, and therefore by $p$ as we can see in Eqs. 1.19 and 1.21 . When $\langle k\rangle$ increases, we can find more differences between the degrees. Another advantage of approximating the degree distribution by a Poisson distribution is that it does not explicitly depend on the network size.

We conclude this section obtaining the expected maximum and minimum degree in a random network. Let us consider a network of $N$ nodes. We define the degree $k_{\max }$ as the value such that the network has at most one node with a degree higher than $k_{\max }$. According to Barabási, $k_{\max }$ means that the area behind the Poisson distribution for all $k \geq k_{\max }$ should be $1 / N$. The area is given by $1-P\left(k_{\max }\right)$, where $P(k)$ is the cumulative degree distribution obtained from $p(k)$. Then, for a Poisson distribution, we have 


$$
\begin{aligned}
1-P\left(k_{\max }\right) & =1-e^{-\langle k\rangle} \sum_{k=0}^{k_{\max }} \frac{\langle k\rangle^{k}}{k !} \\
& =e^{-\langle k\rangle} \sum_{k=k_{\max }+1}^{\infty} \frac{\langle k\rangle^{k}}{k !} \\
& \approx e^{-\langle k\rangle} \frac{\langle k\rangle^{k_{\max }+1}}{\left(k_{\max }+1\right) !}
\end{aligned}
$$

As a consequence, a random network lacks of hubs, or nodes with very high degree. To calculate the value of $k_{\text {min }}$ we follow the same argument used to estimate $k_{\max }$. Here, $k_{\min }$ is the value such that there is, at most, one node with a degree less than $k_{\min }$. Analogously, we have

$$
P\left(k_{\min }-1\right)=e^{-\langle k\rangle} \sum_{k=0}^{k_{\min }-1} \frac{\langle k\rangle^{k}}{k !} .
$$

\subsubsection{Giant connected component}

Consider again Fig. 1.4a. Notice that there are nodes that remain isolated while others have degree $k \geq 1$. When the number of degrees increases, most of the nodes remain in one connected component. Erdös and Rényi obtained the size of the largest connected component of a random network $G(N, p)$. Let us show how this largest connected component can be obtained. Further details can be found in [New18, Ch. 11.5].

The biggest connected component of a random network $G(N, p)$ is the connected set off nodes with the highest number of nodes. It is evident that if $p=0$ the size will be 0 and if $p=1$, then the size will be $N$. In one extreme, the result is independent of the network size, but in the other it is proportional (equal) to the number of nodes. Let us try to determine when there is the change of behavior between these two limiting cases as in [ER59, SR51].

Let us denote by $w$ the average fraction of nodes that do not belong to the giant component. Pick any node $v_{i}$ not in the giant connected component. We have two cases: Firstly, $v_{j}$ is in the giant connected component, so the probability that $v_{i}$ is not connected with $v_{j}$ should be $1-w$. Secondly, $v_{j}$ is not connected in the giant connected component but it is still connected with $v_{i}$, so the probability should be $p w$. Adding both probabilities and considering it for any of the other $n-1$ nodes, we have

$$
w=(1-p+p w)^{N-1} .
$$

Considering Eq. 1.19, we can simplify the previous equation to

$$
w=\left[1-\frac{\langle k\rangle}{N-1}(1-w)\right]^{N-1} .
$$

Then, taking logarithms of both sides we obtain 


$$
\begin{aligned}
\log w & =(N-1) \log \left[1-\frac{\langle k\rangle}{N-1}(1-w)\right] \\
& \simeq-(N-1) \frac{\langle k\rangle}{N-1}(1-w) \\
& =-\langle k\rangle(1-w) .
\end{aligned}
$$

Finally, taking exponentials of both sides again, we have exactly the fraction of nodes that does not belong to the giant component

$$
w=e^{-\langle k\rangle(1-w)} .
$$

Since we are interested in the size of the giant component, we denote it as $G_{\mathrm{C}}$. Since $G_{\mathrm{C}}=1-w$, with $w$ as defined above, the size of giant component will be

$$
G_{\mathrm{C}}=1-e^{-\langle k\rangle G_{\mathrm{C}}} .
$$

We can appreciate that the higher the average degree $\langle k\rangle$, the closer the network is to have a unique connected component. If $\langle k\rangle$ is large enough, then there are two possible solutions: One when $G_{\mathrm{C}}=0$ and another one when $G_{\mathrm{C}}>0$ and only between these values can a giant component be found. We can find the transition value by taking derivatives

$$
\frac{d}{d G_{\mathrm{C}}}\left(1-e^{-\langle k\rangle G_{\mathrm{C}}}\right)=1,
$$

that yields

$$
\langle k\rangle e^{-\langle k\rangle G_{\mathrm{C}}}=1 .
$$

We cannot explicitly express $G_{\mathrm{C}}$ in terms of $\langle k\rangle$. However, we can illustrate this result, like in [New18, Ch. 11.5, Fig. 11.2]. In Fig. 1.7 we have three different curves with a different average degree $\langle k\rangle$. Fig. 1.7a represents the three curves where $y=1-e^{-\langle k\rangle G_{\mathrm{C}}}$ for different average degree value $\langle k\rangle$. The discontinuous black line at the diagonal shows identity equation $y=G_{\mathrm{C}}$, where the intersection gives the solution to Eq. $1.29, G_{C}=1-e^{-\langle k\rangle G_{\mathrm{C}}}$. There is only one intersection for the bottom curve at $G_{\mathrm{C}}=0$, so there is no giant component, while for the top curve, there is a solution at $G_{C}=0.64$. This last solution is represented at the intersection point of the vertical line at $G_{\mathrm{C}}=0.64$ and the identity equation $y=G_{\mathrm{C}}$. The middle curve is precisely at the threshold between the regime where a non-trivial solution for $G_{\mathrm{C}}$ exists and the regime with only the trivial solution $G_{\mathrm{C}}=0$.

In Fig. $1.7 \mathrm{~b}$ we show the resulting solution for the size of the giant component as a function of $\langle k\rangle$. We appreciate a phase transition 


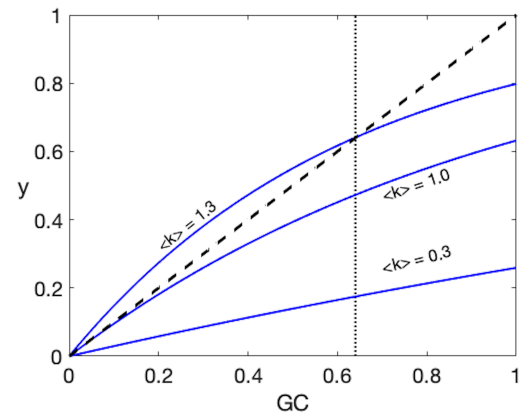

(a)

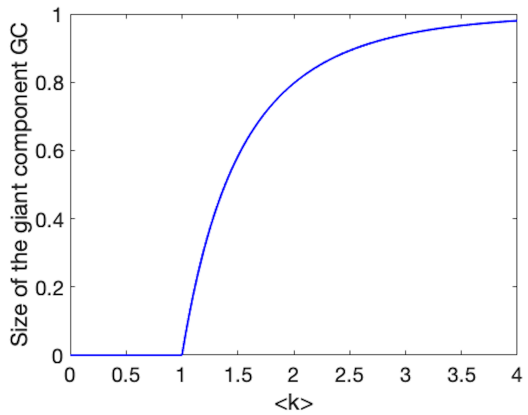

(b)

Fig. 1.7: Graphical solution for the size of the giant component. (a) Representation of the three curves $y=1-e^{-\langle k\rangle G_{C}}$ in terms of $G_{C}$ for $\langle k\rangle=0.3,1$, and 1.3. A phase transition appears when $\langle k\rangle=1$. (b) Phase transition diagram of the relative size of the giant connected component as a function of the average degree. The phase transition is again noticed for $\langle k\rangle=1$.

\subsubsection{Clustering coefficient}

Given an arbitrary node $v_{i}$, its clustering coefficient represents the probability that two of its adjacent nodes are connected by an edge. One of the properties of random networks that differ from other real-world networks is precisely their clustering coefficient since it normally presents values that tend to zero, while the clustering coefficients of many of the networks in real life have higher values, even reaching up to 1 [New18]. In a random network $G(N, p)$, the probability that two of its adjacent nodes are connected by an edge will always be $\frac{\langle k\rangle}{N-1}$, so the local clustering coefficient of $v_{i}$ will be

$$
C_{i}=\frac{\langle k\rangle}{N-1}
$$

To illustrate this result, let us consider the random network of Fig. 1.6a that has $N=2^{15}$ and was generated with probability $p=0.01$. The mean degree will be $\langle k\rangle=327.68$, and hence, the clustering coefficient is $C=0.01$, exactly the probability $p$.

\subsubsection{Path length}

Another one of the most important properties of real networks is the average path length $d$. To calculate it in a random network $G(N, p)$ it is enough to calculate the logarithmic relationship between the average degree and the size of the network New18] through the expression 


$$
d=\frac{N}{\ln \ln \langle k\rangle} .
$$

As an example, we consider the results discussed previously, with $\langle k\rangle=$ 327.68 and $N=2^{15}$ to compute the path length, this is, $d=\ln \left(2^{15}\right) / \ln (327.68)=$ 1.8. We also show in Fig. 1.8 the normalized evolution of the clustering coefficient and the path length Eq. (1.33). We can appreciate that $C_{n}$ tends to 0 and $d$ tends logarithmically to 1 .

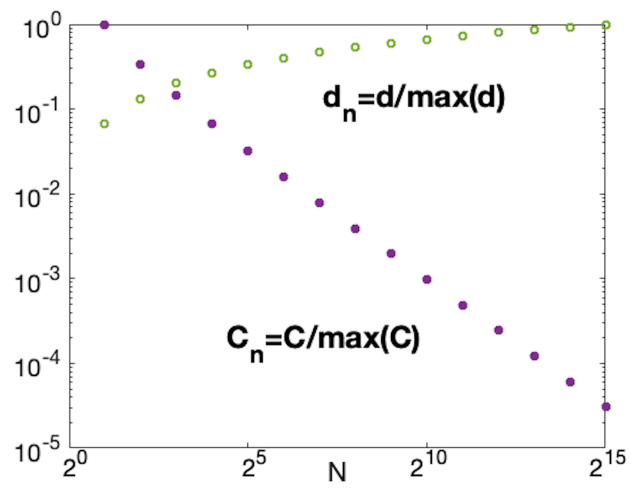

Fig. 1.8: Evolution of the normalized clustering coefficient and path length for random networks of the form $G\left(2^{15}, p\right)$.

\subsubsection{Small world networks}

We have studied random networks in Section 1.5.1, and we saw that one of their most relevant properties is that the clustering coefficient $C_{i}$ tends to zero when the network size grows and that the path length $d$ grows logarithmically, as nodes are added to the network, see Fig. 1.8. However, other real-world network configurations have small path lengths but also high clustering coefficients, see Fig. 1.9. Such networks are called small-world networks and their main characteristics are a small average path and high clustering coefficients.

One of the main examples of this type of networks are social networks. Many social researchers started to work on this topic around 40's, independently of mathematicians. Social scientists were content to study small social communities and weak relationships between people. However, mathematicians found it more interesting to study social networks when the population size was very big. Despite the interest in studying unpredictable connections between people that seemed loosely connected, it was not until 1960 when the social scientist de Sola Pool and the mathematician Kochen provided the 
first advances in the formulation of what we know today about the small world phenomenon [DSPK78, Koc89].

We can consider social networks as networks where people are represented by nodes and their relationships by edges. While the works of de Sola PoolKochen are based on the topologies of the structure of social networks and without contributing much to the literature emanating from random networks, the models Erdös-Rényi abound in its sense. That is, both investigations were polarized, and nothing was known about what was happening between this disciplinary gap $\mathrm{B}^{+} 16$. The preliminary version of DSPK78] inspired Milgram's experiment on social distance, what was later commonly know as the theory of the six degrees of separation or the small world phenomenon Mil67b, Mil67a, MT69. In a few words, this work illustrated that despite the network's size, the distance between any pair of nodes is really small since people are more interconnected than we can think at first sight.

Some drawbacks of Milgram's experiment are the following: It does not favor the quality of the information collected since it does not guarantee that the relationships are minimum distance, favoring many clusters with small numbers of nodes. Random networks cannot predict two important properties that social networks have: (1) the small-world phenomenon (or small mean distances between two randomly selected nodes) and (2) high values of the clustering coefficients. To overcome these deficiencies, Watts and Strogatz presented a new algorithm for generating networks that can satisfy both properties [WS98]. Let us discuss in some detail their model.

Suppose an initial one-dimensional network with $N$ nodes, see Fig. 1.9 These nodes can be arranged in the form of a k-regular ring. That is, each node is connected with $2 k$ of its closest neighbors, i.e., each node $v_{i}$ has degree $2 k$. We denote by $p$ the probability of reconnect each link from one node with any other randomly chosen node. For a network with $p=0$ it can be seen that the connectivity is the same and the degree of each node is still $2 k$, and its clustering coefficient is $C_{i} \sim \frac{3}{4}$. Any non-zero value of $p$ introduces a disorder in the network so that the connectivity is not uniform but still maintaining an average value of $2 k$. This construction allows us to tune the graph between the regularity $p=0$ (Fig. 1.9a) and the disorder $p=1$, see Fig. $1.9 \mathrm{c}$. Therefore, the intermediate region $0<p<1$, see Fig. $1.9 \mathrm{~b}$, is precisely the area where the small-world phenomenon occurs, and where the most interesting and theoretically challenging phenomena occur.

One interpretation of small-world networks is that the distance $d$ between two randomly chosen nodes in a network is short. To illustrate this, let us consider a random network with average degree $\langle k\rangle$. A node in this network has on average $\langle k\rangle$ nodes at distance one $(d=1),\langle k\rangle^{2}$ nodes at distance two $(d=2),\langle k\rangle^{3}$ nodes at distance three $(d=3)$, and so on. Then, the expected number of nodes at a distance $d$ from a given node can be estimated 


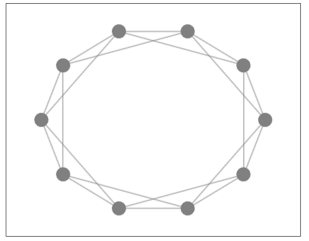

(a) $p=0$

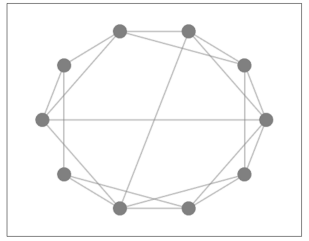

(b) $0<p<1$

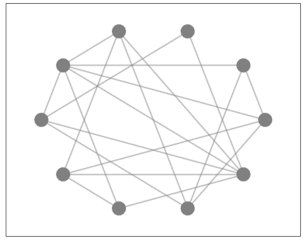

(c) $p=1$

Fig. 1.9: Different configurations to build the small-world networks: (a) A regular ring where each node is connected to their next neighbors. Here, each node has $\langle k\rangle=1 / 2$ with $p=0$. In (b) each node is connected to the rest with probability $0<p<1$. For a small $p$, the network continues with a high clustering coefficient, but the random long-range links can drastically decrease the distances between the nodes $\left[\mathrm{B}^{+} 16\right.$, Box 3.9]. Finally, for $p=1$ the configuration in (c) turns to a random network, as we discussed in Section 1.5 .1

recursively as $\langle k\rangle^{d}=\langle k\rangle \cdot\langle k\rangle^{d-1}$. In this way, the number of nodes at a distance at most $d$ from a given node can be estimated as

$$
N(d) \approx 1+\langle k\rangle+\langle k\rangle^{2}+\cdots+\langle k\rangle^{d}=\frac{\langle k\rangle^{d+1}}{\langle k\rangle-1} .
$$

If $d_{\max }$ is the network diameter, then $N\left(d_{\max }\right) \approx N$. For values of $\langle k\rangle \ll 1$, we can neglect the -1 in the numerator and denominator, and taking logarithms we get

$$
d_{\max } \approx \frac{\log (N)}{\log (\langle k\rangle)} .
$$

Some remarks should be given concerning this last equation. On the one hand, since the nodes at a distance $d_{\max }$ are usually very few, the estimation in Eq. 1.35 works quite well as an approximation of the average path length $\langle d\rangle$. On the other hand, since $\log (N) \ll N$, we have that the dependency of $\langle d\rangle$ in $\ln (N)$ implies that the distances in a random network are several orders of magnitude lower than the network size. Consequently, by small in the small world phenomenon, we mean that the average path length (or diameter) depends logarithmically on the size of the network. Therefore, small world means that $\langle d\rangle$ is proportional to $\log (N)$, rather than $N$ or some power of $N$.

To calculate the local clustering of a node $v_{i}$, we must first estimate the number of edges $L_{i}$ between their $k_{i}$ adjacent nodes. We know that the probability that the neighbors of a given node are linked to each other is $p$ and there are $k_{i}\left(k_{i}-1\right) / 2$ possible links between the $k_{i}$ adjacent nodes to $v_{i}$. 
Therefore, the expected value of $L_{i}$ will be $\left\langle L_{i}\right\rangle=p \frac{k_{i}\left(k_{i}-1\right)}{2}$. Hence, the local clustering $C_{i}$ will be given by

$$
C_{i}=\frac{2\left\langle L_{i}\right\rangle}{k_{i}\left(k_{i}-1\right)}=p=\frac{\langle k\rangle}{N} .
$$

From this last equation, we can conclude that if $\langle k\rangle$ is fixed, then the probability $p$ (and the local clustering coefficient $C_{i}$ ) tend to zero as $N$ grows, at the rate $1 / N$. Besides, despite being a local measure, the local clustering coefficient depends heavily on the network size $N$.

\subsubsection{Scale-Free Networks}

In this section, we will describe the scale-free networks proposed by Barabási and Albert in 1999. At that time, along with other researchers, they focused on projects to map the World Wide Web $(W W W)$, where the documents are represented by nodes and the hyperlinks between documents by edges. In collaboration with Jeong, they mapped the domain of the University of Notre Dame nd.edu, which at that time had 300,000 documents and 1.5 million links between them $\left[\mathrm{B}^{+} 16\right.$, Video 4.1]. The goal was to approximate the properties of random networks using Poisson distributions. They expected that the topology of the web would behave like a random social network because each document contained information from personal and professional searches.

However, when looking at the results, they realized that certain pages were highly connected to others despite the WWW network's apparent randomness. This phenomenon is contrary to what should happen in a random network because the presence of these hyperlink containers produces a series of high clusters and, which is in disagreement with Eq. 1.32 already discussed in Section 1.5.1.3.

This study revealed the existence of very few pages that were highly connected while most of the pages remained with very few links. The existence of hubs (nodes with an exceptionally large number of edges) led them to verify that if a large number of nodes (more than 80\%) were disconnected, the network remained connected. However, if some nodes with many connections were disconnected (between 5 and $10 \%$, approximately), then the network was disconnected. The most exciting point about this property is that it also holds for some other networks such as sexually transmitted diseases $\left[\mathrm{LEA}^{+}\right.$01, phone books $\mathrm{OSH}^{+}$07], bibliographic citations between members of the academic community New01, technological alliances, coappearance of film actors BF02, neural synapses [CH03, contacts between people in an organization $\mathrm{WWA}^{+} 08$ ], food chains [PLC91, connections between organisms linked to metabolism or regulatory proteins $\mathrm{RSM}^{+} 02$ or the spread of diseases and computer viruses [PSV01]. 
The presence of hubs allowed the emergence of a new type of networks called scale-free networks. We will see that the degree distribution of any of these networks follows a power-law of the form

$$
p_{k}=C k^{-\gamma}
$$

with $C, \gamma>0$.

Networks showing such degree distribution show some other properties, such as:

1. The exponent $-\gamma$ in the power-law $\left(k^{-\gamma}\right)$ is usually between 2 and 3 .

2. Any node is connected to any other node with very few degrees of separation (small-world phenomenon). In fact, distances are even smaller than in random networks, due to the ultra-small regime of scale-free networks, see $\mathrm{B}^{+} 16$, Sec. 4.6$]$.

3. Existence of nodes whose connectivity value exceeds the average number several times, which is not typical of random networks.

4. They present hierarchical modularity, in the sense that several small communities form bigger communities, which recombine into even bigger communities. This can be appreciated when looking at the dependence of the node clustering coefficient of its degree, which usually follows the law $C_{i} \approx i^{-1}$. This property will be explained in Section 1.5.4.

Due to the singular distribution of scale-free networks, many of the statistical methods such as sampling, analysis of variance, generalization, correlation coefficients are not relevant to treat them. The reason is that these methods implicitly involve normal distributions, which does not occur in these networks. However, we will try to outline some notions about these networks.

\subsubsection{Power-laws and scale-free networks}

We consider, again, an arbitrary and undirected network $G=(V, E)$, with degree distribution $p(k)$. Mathematically, the scale-free networks show an asymptotic behavior that is determined by Eq. (1.37). The probability $p(k)$ that a node has degree $k$ is inversely proportional to $k$ power to some exponent $\gamma>0, p(k) \propto k^{-\gamma}$.

In general, in the real-life and nature examples, we find $2<\gamma<3$. The power-law degree distribution is associated with a preference for attachment and far away from being random. Therefore, a Poisson distribution presents a poor adjustment when fitting a power-law distribution, mainly due to the hubs. However, on a logarithmic axis scale $(\log -\log )$, the data points form an approximate straight line.

For representing power-law distributions, it is better to take logarithms to both sides of Eq. (1.37). Then, one gets that $\log p(k)$ depends linearly on $\log k$, whose coefficient (slope) is the value of the exponent $\gamma$ 


$$
\log p(k)=-\gamma \log k+\log (C) .
$$

We can see that this distribution depends on the $\gamma$ power of $k$, and this is the reason why this type of distribution is called a power-law. Since $p(k)$ represents the fraction of nodes with degree $k$, this means that $p(k)$ is the probability of the total fraction of nodes with degree $k$, therefore

$$
\sum_{k=0}^{\infty} p(k)=1
$$

However, for $k=0$, Eq. 1.37 diverges because $p(0)$ tends to $\infty$ and we know that all probabilities must lay between 0 and 1 . This leads us to think that the power-law distribution has some minimum degree $k_{\min }$. Let us suppose that $k_{\min } \geq 1$, which means that there are no nodes with zero degrees so that $p(0)=0$, when substituting in Eq. 1.39 , we get

$$
C \sum_{k=1}^{\infty} k^{-\gamma}=1
$$

or

$$
C=\frac{1}{\sum_{k=1}^{\infty} k^{-\gamma}}=\frac{1}{\zeta(\gamma)},
$$

where $\zeta(\gamma)=\sum_{k=1}^{\infty} k^{-\gamma}$ is the Riemann zeta function and therefore, for all $k>0$ and assuming $p_{0}=0$, the correctly normalized power-law distribution will be

$$
p(k)=\frac{k^{-\gamma}}{\zeta(\gamma)} .
$$

It is important to highlight that the expression in Eq. 1.42 still diverges for the value of $k=0$. The $k_{\min }$ is exactly the point from which the tail of the distribution becomes heavy. Often only the heavy tailed part of the distribution contains the relevant information of the network, particularly it contains the greatest number of degrees [CSN09. For this reason, we must normalize the fraction of nodes with degree $k$ by the minimum value of the degrees, that is, $k_{\min }$ and we get

$$
p(k)=\frac{k^{-\gamma}}{\sum_{k=k_{\min }}^{\infty} k^{-\gamma}}=\frac{k^{-\gamma}}{\zeta\left(\gamma, k_{\min }\right)},
$$

where $\zeta\left(\gamma, k_{\min }\right)=\sum_{k=k_{\min }}^{\infty} k^{-\gamma}$ is the so-called generalized zeta function. If we consider a $k_{\min } \geq 1$, the sum over all $k$ is well approximated by the integral and therefore the normalization constant $C$ can be written as

$$
C \simeq \frac{1}{\int_{k_{\min }}^{\infty} k^{-\gamma} d k}=(\gamma-1) k_{\min }^{\gamma-1},
$$


or

$$
p(k) \simeq \frac{\gamma-1}{k_{\min }}\left(\frac{k}{k_{\min }}\right)^{-\gamma} .
$$

In the same way, the cumulative distribution function represented can be written as

$$
P(k)=\left(\frac{k}{k_{\min }}\right)^{-(\gamma-1)} .
$$

\subsubsection{Size of hubs}

There are networks whose number of nodes is huge. For instance, our brain has approximately $10^{11}$ neurons and the WWW, $10^{12}$ webpages, see Table 1.4.1. However, in all the networks that the human has discovered, there is finiteness. This means that we can count the number of nodes and connections in these networks, particularly in scale-free networks. Let us estimate the degree of the hubs. Suppose that there is a value $k_{\max }$ from which it is expected that at most, there will be a vertex with a degree greater than

$k_{\max }$. From the Eq. 1.46 and taking into account that $\int_{k_{\max }}^{\infty} p_{k} d k=\frac{1}{N}$, we obtain that

$$
k_{\max }=k_{\min } N^{\frac{1}{\gamma-1}} .
$$

The previous expression allows to conclude that the size of the hub in a network is directly proportional to the number of nodes and connections. That is, the larger a network is, the greater the degree of its largest hub will be.

\subsubsection{Hierarchical networks}

Hierarchical networks or modular networks are a type of complex network whose construction is based on the dense interconnection between nodes, see Fig. 1.10. The modularity characteristic is due to the underlying property in this type of network that has to do with preferential attachment. That is, nodes prefer to connect to those of the same community rather than those of another $\left[\mathrm{B}^{+} 16\right.$, Ch. 9.1].

We illustrate here two of the main types of hierarchical networks:

1. Social networks. A very particular characteristic of social networks is that they are full of communities, which are easily identifiable. This makes the study of social networks topologies of vital importance. Zachary was the first one who applied graph theory methods in order to identify these communities [Zac77]. This social network has been studied due to a conflict between the club administrator, John A., and the instructor, Mr. Hi. This conflict caused the club to split into two equally proportioned parts, see Fig. 1.11a, which could be identified after studying the applying Ford-Fulkerson algorithm to the social network obtained measuring the affinity between their members. 


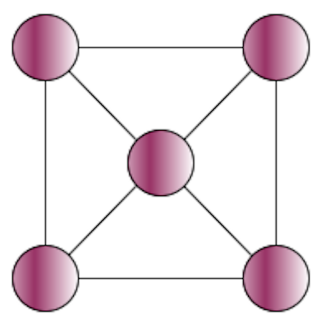

(a)

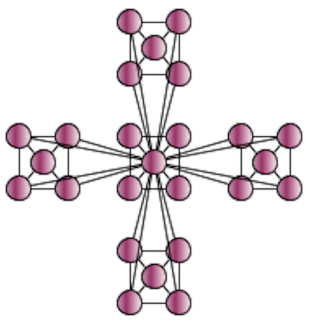

(b)

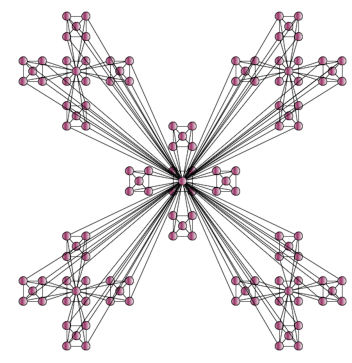

(c)

Fig. 1.10: Representation of some hierarchical networks. Panel (a) shows a densely connected center-module with 5 nodes and 8 edges. (b) Four replicas of the center-module, where one can see that peripheral nodes are connected with the center node of the center-module. This structure has 25 nodes and 56 edges. (c) Twenty-five replicas of the center-module. This structure has 125 nodes and 344 edges.

2. Biological Networks. In 1999, L.H. Hartwell argued that "biology must move beyond its focus on single genes. It must explore instead how groups of molecules form functional modules to carry out specific cellular functions" [HHLM99. This argument caught the attention of E. Ravasz et. al., who led the first attempt to identify these modules in metabolic networks systematically $\left[\mathrm{RSM}^{+} 02\right]$, see Fig. $1.11 \mathrm{~b}$. They made the following fundamental hypothesis: A network's community structure is uniquely encoded in its wiring diagram. It was possible by constructing a model that allowed the identification of sub-groups of molecules that, being interconnected, formed communities.

The concept of modularity is the main characteristic of this type of network. It is closely related to the emergency property, which characterizes complex networks that expand as nodes are added to the network. The modularity of this network implies that: 1) nodes have a preferential attachment, and 2) the clustering coefficient is high because each module is densely connected, both with the module that generates it, as well as with the potentials modules that it generates.

\subsubsection{Degree distribution}

One of the essential measures in the study of complex networks is the degree distribution. We will show an example of how to study the degree distribution in one particular case, starting from one of the main characteristics of scalefree networks: the hubs.

Let us consider Fig. 1.12, as it is done in $\left[\mathrm{B}^{+} 16\right.$, Adv. Top. 9.A]. We identify the center of one of the smallest modules as hubs, and they will be 


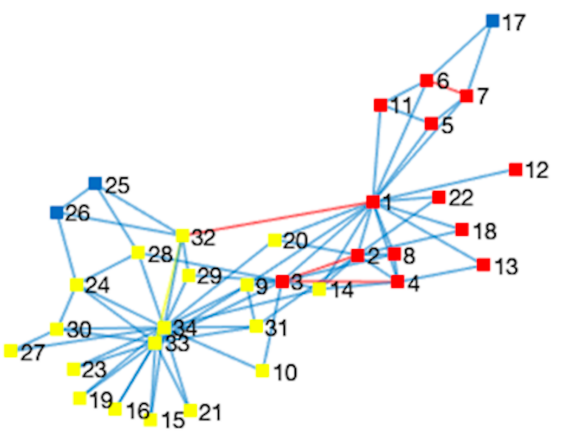

(a)

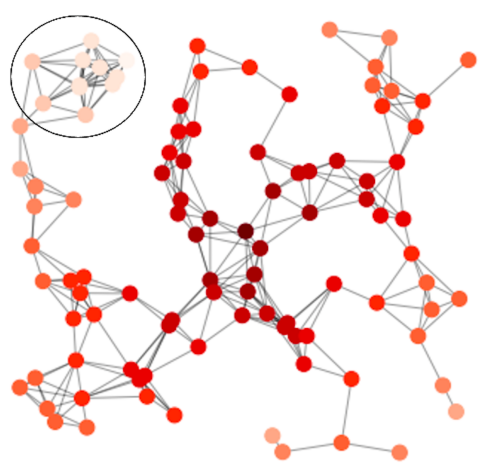

(b)

Fig. 1.11: Representation of two examples of hierarchical networks. (a) shows Zachary's Karate social network in which the color nodes represent two communities. This network has 34 nodes and 78 edges. (b) shows an example of a metabolic network with 100 nodes and 311 edges. Each color represents a different community.

denoted by $H$. For example, we call $H_{n}$ to the central node of the hierarchical network. Then, we consider one of its repetitions, which we call $H_{n-1}$ to its central node. Next, we consider the peripheral module, considering its central node, which we call $H_{n-2}$. We repeat this process for all peripheral branches throughout the network.

Fig. 1.12 is built for 4 iterations. For convenience, let us consider a hierarchical network obtained by this recursive process after $n$ iterations. After the $n$-th iteration, the degree of the hub $H_{i}$, denoted by $k_{n}\left(H_{i}\right)$ is

$$
k_{n}\left(H_{i}\right)=\sum_{l=1}^{i} 4^{l}=\frac{4}{3}(4 i-1)
$$

Let us now count the number of modules in the network. We have 4 modules for $i=n-1,4 \cdot 5$ modules for $i=n-2$ and so on, until $4 \cdot 5^{n-2}$ for the case $i=1$. Recursively, the number of $H_{i}$ modules hubs with degree $k_{n}\left(H_{i}\right)$ will be $N_{n}\left(H_{i}\right)=4 \cdot 5^{n-i-1}$. Substituting in Eq. 1.48, we have

$$
\log \left(N_{n}\left(H_{i}\right)\right)=C_{n}-i \cdot \log (5),
$$

with $C_{n}=\log (4)+(n-1) \cdot \log (5)$. Besides, we can also approximate $\log \left(k_{n}\left(H_{i}\right)\right) \approx i \cdot \log (4)+\log (4 / 3)$. Combining both expressions, we get

$$
\log \left(N_{n}\left(H_{i}\right)\right)=C_{n}^{\prime}-\log \left(k_{n}\left(H_{i}\right)\right) \frac{\log (5)}{\log (4)},
$$




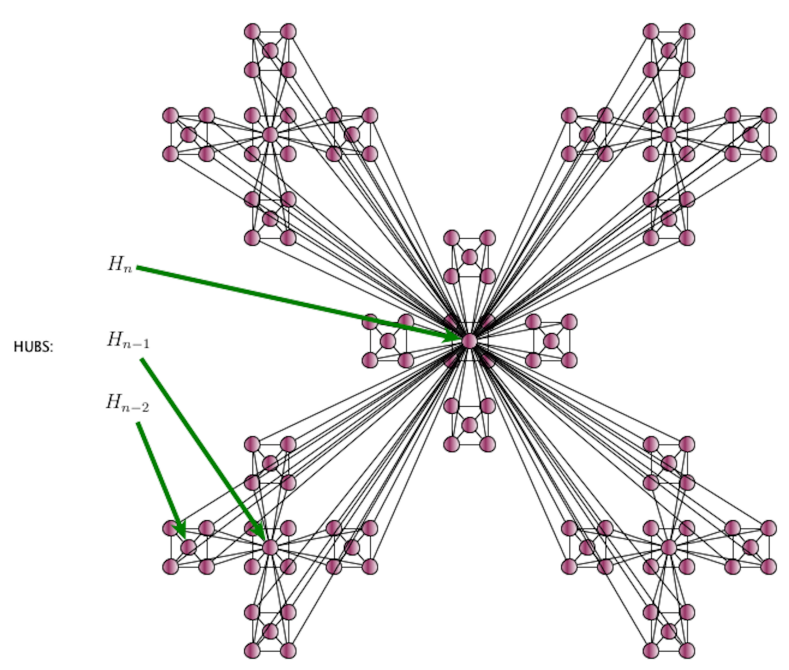

Fig. 1.12: Computing the Degree Distribution like [ $\mathrm{B}^{+} 16$, Fig. 9.35].

for some constant $C_{n}^{\prime}$ or, simplifying the notation $k_{n}\left(H_{i}\right)$ by $k_{i}$, we have

$$
N_{n}\left(H_{i}\right) \approx \log \left(k_{i}^{-\frac{\log (5)}{\log (4)}}\right) .
$$

For computing the degree distribution, it is necessary to normalize Eq. (1.51) as follows

$$
p_{k_{i}} \approx \frac{N_{n}\left(H_{i}\right)}{k_{i+1}-k_{i}} \approx k_{i}^{-\gamma} .
$$

Since $k_{i+1}-k_{i}=\sum_{l=1}^{i+1} 4^{l}-\sum_{l=1}^{i} 4^{l}=4^{i+1}=3 k_{i}+4$, we obtain

$$
p_{k_{i}}=\frac{k_{i}^{-\frac{\log (5)}{\log (4)}}}{3 k_{i}+4} \approx k_{i}^{-1-\frac{\log (5)}{\log (4)}},
$$

and $\gamma=1+\frac{\ln 5}{\ln 4}=2.16$, satisfying that $2<\gamma<3$, that is a characteristic parameter for scale-free networks.

\subsubsection{Hierarchical clustering}

Let us consider again Fig. 1.10. Here, the network is built up of a number of small cross-shaped sub-modules Fig. 1.10a which in turn are divided into smaller modules with 5 nodes Figs. $1.10 \mathrm{~b}$ and $1.10 \mathrm{c}$ The measure that captures the modularity in this type of networks is the clustering coefficient $C_{i}$ of the node $v_{i}$, expressed in terms of the behavior of its $k_{i}$ adjacent nodes. From Eq. 1.51), we can obtain the clustering coefficient of the hubs $H_{i}$ 


$$
C\left(H_{i}\right)=\frac{2 k_{i}}{k_{i}\left(k_{i}-1\right)}=\frac{2}{k_{i}-1} .
$$

So that, we have that hubs satisfy that $C(i) \approx \frac{2}{i}$. Therefore, we can appreciate a property that usually holds in scale-free networks: the higher the degree of a node is, the lower its clustering coefficient is.

\subsection{Network in number theory}

Numbers represent absolute reality.

Pythagoras.

Modeling relations between numbers through networks seems to be an exciting idea that captures the scientific community's attention dedicated to studying complex systems. There is an interest in checking whether the behavior observed in networks associated with real-life processes is also replied in networks associated with abstracts elements such as numbers and with different connectivity relationships between them.

We know the human capacity to create is extensive and is often unlimited Ara10, and one of his most commendable creations is the numbers Bor66. Since numbers were discovered, humans have used them to number and count, coming up with clever and fun ways to do it Str12, Loc09, as if it were a game, going from one infinity to another [Dau90. The wealth and properties that are inherent in numbers have allowed the creation of what is now known as Number Theory, which is the branch of mathematics that is responsible for studying the properties of integers [Apo13, Hist. Introd.]. Precisely, this section aims to study the relationship between number theory and network theory. These two fields have found a commonplace in the study of networks whose elements are numbers that are linked through some mathematical relationship.

\subsubsection{Divisibility networks}

Zhou and Wang were the first ones to study networks that emerge from number theory concepts, such as the divisibility network of the natural numbers in [ZWHC06]. In this work, they represented the nodes as natural numbers, and two nodes $n, m \in \mathbb{N}$ were connected by a directed edge if $n$ divides to $m$. One of their most important results is that this network has a large clustering coefficient $C \approx 0.34$, which is almost not sensitive to the network size. Besides, they also show that the average distance $\langle d\rangle$ between a pair of nodes is upper bounded in contrast to small-world networks [WS98, in which there 
is a lower bound for $\langle d\rangle$. Some other interesting results are that the network posses a hierarchical architecture and that the degree distribution is scalefree of the form $p(k) \sim k^{-\gamma}$. Fig. 1.13 shows a representation of the natural number divisibility structure.

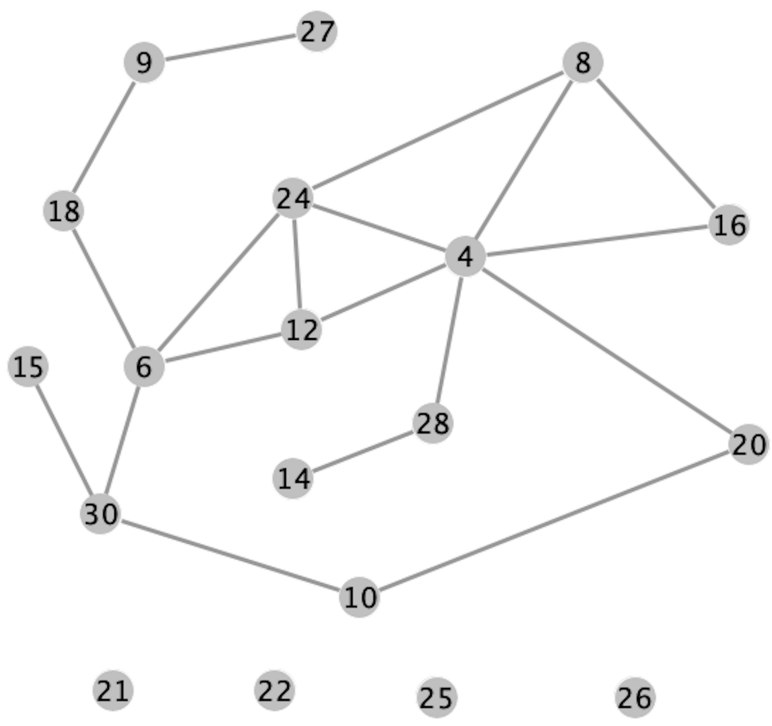

Fig. 1.13: Network representation with 19 nodes and 19 edges. There are 5 disjoint components. The relationship is due to the divisibility patterns $\bmod 0$.

Shekatkar et. al., studied the undirected network of divisibility of natural numbers $(\mathbb{N})$. Here, nodes are the natural numbers and two nodes $a, b$ are connected by an edge provided that $a \mid b$ or $b \mid a \bmod (0)$ [SBA15. The structure studied consisted of a network in constant growth, from time $t=1$ that represents a single node, i.e., $N=1$, up to time $t=2^{25}$, which consisted of a network of $N=33.554 .432$ nodes. They showed that its degree distribution is scale-free. Applying the maximum likelihood method, they found that the scaling-index $\gamma \sim 2$. Another interesting result is that the local clustering $C_{k}$ is not stationary, which has allowed the emergence of a new property that they called similarity stretching with respect to a value $C_{\Delta}=0$. They also showed that the average degree grows logarithmically as a function of $N$, that is, $\langle k\rangle_{n} \sim 2 \log (N)-1.6912$. We illustrate this in Fig. 1.14

Yuan et. al., $\left[\mathrm{YZW}^{+} 14\right]$ and Yan et. al., [YWCS16] studied the relationship between arithmetic congruences of natural numbers and layered networks 


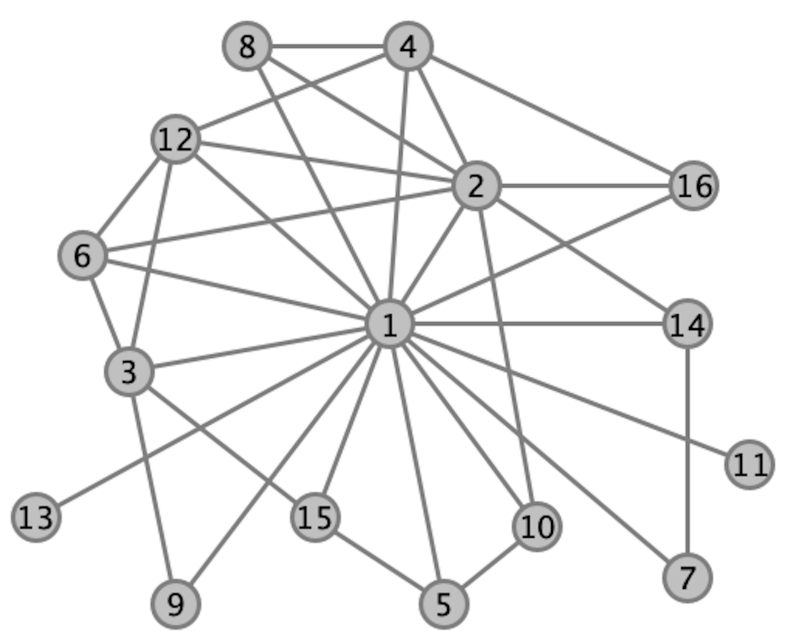

Fig. 1.14: Divisibility network representation for the first 16 natural numbers.

of the form $G(r>0, N)$, with $N$ the number of nodes. There, the connectivity relationship between the nodes $v_{i}, v_{j}$ is established by the edge $e_{i j}$, such that, $j \equiv r \bmod (i)$. These directed networks satisfy that if $r$ is large, then the layers are dispersed. Each layer has only $N-r$ nodes interconnected. The parameter $r$ determines the structure of the congruence network $G$. For example, if $r=0$, then the congruence network reduces to a divisibility network, equal to the networks already studied in [ZWHC06, SBA15]. Besides, the authors provided an analytical expression of the distribution of degrees when the network $G$ is very large.

$$
p(k)=\frac{1}{k(k+1)},
$$

and therefore, for very large $k$, the degree distribution becomes $p_{k} \approx k^{-2}$, obtaining as a result, a scale-free network. Like the result of Shekatkar, et. al., the average degree grows logarithmically with $N$ following the next expression

$$
\langle k\rangle \approx \log (N-r)+H
$$

where

$$
H=2 C-1-\frac{\sum_{i=1}^{r}\left\lfloor\frac{N-r}{i}\right\rfloor}{N-r},
$$

is a constant. When $r$ is very large $H \approx C-1-\log (r)$, with $C \approx 0.57721$ being the Euler constant. 
Rajans and Ambika continued the study of divisibility patterns between the natural numbers in RA20. In this recent work, they showed a new approach to study, analytically, some of the most relevant topologies of complex network models, such as the nodes degrees, the local and global clustering coefficients, the geodesic distances, and the centrality of a node. All these analyses are expressed in terms of the floor and divisors functions $\lfloor\cdot\rfloor$, and $s(n)$, respectively. Besides, given that the primes are distributed in a nonhomogeneous way in the set of natural numbers [DGGB11], the authors derive analytical expressions from verifying how the primes are in the degrees distribution related to each node. For instance, they notice that the node degree $k_{n}$ can be approximated, analytically, as

$$
k_{n}=\lfloor N / n\rfloor+s(n)-2,
$$

where $n \in V$, such as: $n=\prod_{i=1}^{k} p_{i}^{\lambda_{i}}$, and $s(n)=\prod_{i=1}^{k} p_{i}^{\lambda_{i}+1}$, here $\lambda \in \mathbb{Z}^{+}$. The proof is in [RA20, Th. 1]. The Eq. (1.58) means that the distribution of the degrees throughout the network is directly related to the floor function divisors function (see RA20, Fig. 2]). They also show that the density of the subnetwork of size $N$ can be approximated by $N^{-3 / 4}$, following a power-law.

Corso et. al., developed a network of the form $G=(V, E)$ whose nodes are natural numbers $V=\mathbb{N}$ and two numbers are connected following the next rule: Given $a, b \in \mathbb{N}$, we say that $a$ is connected with $b$, if and only if, they share a common prime factor $p_{l}$ Cor04. These networks have a high clustering coefficient and an average path length that is close to 1.8.

Luque, Miramontes, and Lacasa studied the divisibility patterns of natural numbers [LML08, but with a different approach from that studied in [ZWHC06, SBA15]. They were looking for a quite simple and general process in which a scale-free degree distribution can induce the onset of criticality in the dynamics of self-organized systems. They considered an iterative driven and dissipative process in which integers are constantly introduced and removed from it. First, we recall that a primitive set of natural numbers, i.e., none of the elements of $V$ divides exactly another. We start with a primitive set $V$ of $n$ elements extracted from the numbers between 2 and $m$, with $n<m$. The rest of the numbers remain in a pool. We pick a new number from the pool at random, and we introduce it in $V$. If the set is still primitive, we pick another number from the pool. If the set is not primitive, either the number added divides or is divided by $s$ elements. So, we retire them and repeat the process. With this simple process, Luque et. al., showed a general mechanism by which simple dynamics running on networks become self-organizing critical for unscaled topologies.

García-Pérez et. al., studied the relationship between networks and stochastic processes theories, through the architecture of prime and composite numbers GPSB14. They proposed a non-parametric non-Markovian dynamical 
model that naturally generates primes randomly and study their relation with composite numbers. The stochastic process is non-Markovian, because the probability of a number being a prime depends on the probabilistic process on all $\mathbb{Z}$, that is, $P_{N}$. This probability follows a dichotomous selection process that is defined for $k=2, \ldots, N$ as

$$
n_{k}= \begin{cases}1, & \text { if } k \text { is prime } \\ 0, & \text { otherwise }\end{cases}
$$

Hence, we can write $P_{N}$ as

$$
P_{N}=\left\langle n_{N}\right\rangle=\sum_{n_{2}=0}^{1} \cdots \sum_{n_{N}=0}^{1} n_{N} \rho\left(n_{2}, \cdots, n_{N}\right),
$$

where $\left\langle n_{N}\right\rangle$ is the statistical average and $\rho\left(n_{2}, \ldots, n_{N}\right)$ is the joint probability of the particular sequence $\left(n_{2}, \ldots, n_{N}\right)$.

Besides, they indicate that their model refines, to a great extent, the statistical model proposed by Cramer, related to the existing gap between consecutive prime numbers. Besides, this model allows a better understanding of the counting function of the prime numbers, proposing a stochastic function in which, given an integer $z \in \mathbb{Z}$, the probability of know how many primes there are, such that $|p|<|z|$.

Natural numbers can be represented by a weighted bipartite network. Its construction is as follows: Applying the Arithmetic Fundamental Theorem Apo13, Ros13, we can decompose any natural number as a unqiue product of prime factors. Each composite number is connected with the prime factors appearing in its decomposition, with weights according to the number of times that this prime number appears in the factorization. It allow us to build Fig. 1.15 where we descompose a composite number as $n_{c}=p_{1}^{\lambda_{1}} \cdot p_{2}^{\lambda_{2}} \cdots p_{k}^{\lambda_{k}} \cdots$, where $p_{i}$ is the $i$-th prime and $\lambda_{i}$ are non-negative integers. 


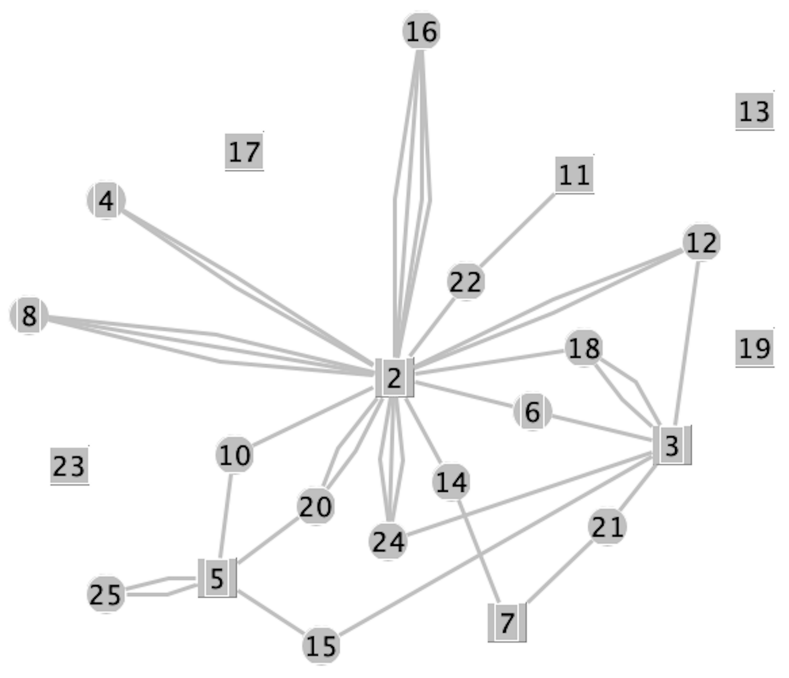

Fig. 1.15: Bipartite network of natural number of side $n=25$, like GPSB14, Figure 1]. Here we can observe the decomposition of the natural numbers in composite and prime factors. Circled nodes represent composite numbers, and squared nodes represent prime numbers.

One of the most important results in this work is that the for a network size $N$, the degree distribution $P\left(k_{p}\right)$, that a randomly chosen prime is connected to $k_{p}$ different composite numbers, is given by

$$
P\left(k_{p}\right)=\frac{\pi\left(\frac{N}{k_{p}+1}\right)-\pi\left(\frac{N}{k_{p}+2}\right)}{\pi(N)},
$$

where $\pi(N) \sim \frac{N}{\ln N}$ is the prime counting function [GPSB14, Eq. (2)], $k_{p}=$ $0,1, \cdots,\left\lfloor\frac{N}{2}\right\rfloor$ and $\lfloor x\rfloor$ denotes the floor function. Another very important result is that, applying the prime number theorem, we get that the degree distribution behaves following a power-law of the form $P\left(k_{p}\right) \sim k_{p}-2$.

\subsubsection{Network analyses of conjectures in number theory}

Some of the most well-known conjectures in number theory have been revisited from the perspective of network science. We briefly outline here the main existing results. 


\subsubsection{The Collatz Conjecture}

Emmert-Streib analyzed the Collatz conjecture from complex network theory perspective ES13. The Collatz sequence's conjecture was enunciated by the Collatz in 1937, and it has not been resolved yet. The conjecture states that "every positive integer has an orbit under the function $T$ defined as

$$
T(n)=\left\{\begin{array}{l}
1, \quad \text { if } n=1, \\
3 n+1, \quad \text { if } n \text { odd is and } n>1, \\
\frac{n}{2}, \quad \text { if } n \text { is even. }
\end{array}\right.
$$

which will always reach 1 and, therefore, it will also reaches the cycle $(4,2,1)$ Lag85.

Apart from Collatz graphs [ES13, Fig. 1], they propose the study of Collatz step graphs, that are defined as follows: Given $n \in \mathbb{N}$, let us define the function $\lambda(n)=t$ if $T^{t}(n)=1$, for all $n>1$. Then, we define a second one $\theta: \mathbb{N} \rightarrow$ $\mathbb{N}^{n-1}$ by $n \theta(n, T)=(\lambda(2), \ldots, \lambda(n))$ for $n>1$. Let us construct the Collatz step sequence graph, for brevity denoted as $G_{C S}$, as follows: The set of nodes will be given by the natural numbers $m$ such that $m$ is equal to the $i$-th component of $\Theta(n)$ for some $1 \leq i \leq n-1$. Two nodes $m$ and $k$ are connected if exists some $i$ such that $m=\Theta_{i}(n)$ and $k=\Theta_{i+1}(n)$, see [ES13, Fig. 2]. If we count how many $i$ 's provide this equality, we can even generate a weighted graph.

The authors claim that this network was motivated after studying the mechanism of a biological cell. Collatz orbits are very similar to the process that occurs in a cell, when DNA follows a linear sequence until it is transcribed into a mRNAs, which is subsequently translated into proteins that interact with each other, as well as other types of gene structures [Zha09, ESG11]. After studying these networks, they conclude the following:

1. There are clear and subtle differences between the orbits of the odd and even natural numbers.

2. The average number of steps until an orbit reaches 1 [ES13, Eq. 13] is logarithmically approximated with the size of $n$.

3. The distribution of weighted degrees behaves, asymptotically, like a power-law distribution of the form $p(k) \sim k^{-0.92}$. It is important to note that the value of the exponent is not affected by the number of nodes and, therefore, is invariant to the size of the network.

4. If $n \rightarrow \infty$, the average path length $L(n)$ tends to

$$
L^{\infty}= \begin{cases}3.069 \pm 0.006, & \text { if } G_{C S} \text { is undirected } \\ 4.134 \pm 0.009, & \text { if } G_{C S} \text { is directed }\end{cases}
$$

5. If $n \rightarrow \infty$, then the global clustering coefficient tends to $0.609 \pm 0.004$. 


\subsubsection{The Goldbach Strong Conjecture}

Chandra et. al., offered us one fascinating result by relating the Goldbach Strong Conjecture (GSC) SL84 with network science CD05. The beauty of his work is to establish a probability function that establishes the minimum distance between pairs of possible primes that result in an even number. Recall that the GSC, which was proposed by Goldbach in 1742, states that "every even number $\left(n_{e}\right)$ greater than 4 is the sum of two prime numbers $p_{1}, p_{2}$ ", that is: $n_{e}=p_{1}+p_{2}$, and Goldbach's weak conjecture (GWC) states that "every odd number $\left(n_{o}\right)$ greater than 5 can be written as the sum of three primes $p_{1}, p_{2}, p_{3}$ ", that is: $n_{o}=p_{1}+p_{2}+p_{3}$ [Sch40]. GWC was proved in 2015 by Helfgott Hel13. Chandra et. al., started from GSC, where nodes were prime numbers and for every even number $n$, we put an edge between the component primes $p$ and $q$ with probability $|p-q|^{\alpha}$, with $-\infty<\alpha<\infty$.

For example, let us consider $n=34$. We can decompose it into the following sums of two primes: $3+31,5+29$ and $11+23$. Then, we define $\Delta=|p-q|$, that is $\Delta=28,24,12$. The possible edges are $L=\{(3,31),(5,29),(11,23)\}$. We define a variable $s=\sum_{i=1}^{k} \Delta_{i}^{\alpha}$, i.e. $s=28^{\alpha}+24^{\alpha}+12^{\alpha}$. Then, we can define $p_{i}=\frac{\Delta_{i}^{\alpha}}{s}$, as the probability that each edge connects the primes $(p, q)$; in our example, it would be $: p_{1}=\frac{28^{\alpha}}{s}, p_{2}=\frac{24^{\alpha}}{s}$ and $p_{3}=\frac{12^{\alpha}}{s}$. Given that $p_{1}+p_{2}+p_{3}=1$, a pair of primes is chosen that belongs to the interval $[0,1]$. Then, for $M$ even numbers, we will have $M$ edges because only one edge is placed for each pair of primes $(p, q)$, but the number of nodes will be smaller $N<M$. If $\alpha=0$, then the choice of the pair $(p, q)$ is independent of the value of $\Delta$, while if $\alpha \neq 0$, then the edge that goes from $p$ to $q$ must meet the probability that the distance is minimal. As results, the authors report that if $\alpha>-1.8$ the network that is formed presents a small-world topology, i.e., the average shortest distance is small (increases only logarithmically with the size of the network), but the clustering coefficient is high compared to the random network, and if $\alpha<-1.8$, the network is regular [WS98.

\subsubsection{The Erdös-Straus Conjecture}

Mondreti, et. al., in , offers us another interesting result in which a number theory problem can be modeled and verified using complex network theory techniques Mon19. In his work, Mondreti studied the famous Erdös-Straus Conjecture. This conjecture states that "for any positive integer $n \geq 2$, the Diophantine Equation (DE)

$$
\frac{4}{n}=\frac{1}{x}+\frac{1}{y}+\frac{1}{z}
$$

has a solution where $x, y, z$ are also positive integers". 
To the aim of building an increasing family of networks $G_{n}$ associated to Graph of the Edrös-Straus Conjeture. The authors build function $S(n)$ that generates an unordered set of integers that could be part of the solution to Eq. 1.64). With this in mind, they generate another function $D_{n}=\{k \in \mathbb{N}$ : $k \leq n\}$ that contains all the triplets of solutions $x, y, z$, which correspond to the denominators of the DE. From the two previous functions $S_{n}$ and $D_{n}$, it is easy to determine the set of nodes, which is defined as

$$
V_{n}=D_{n} \bigcup_{i \in D_{n}} S_{i} .
$$

Now, the relationships between nodes are established by the connections between the unique positive integer solutions to Eq. (1.64), that is,

$$
E_{n}=\left\{(s, t): \forall t \in S_{s} \text { for each } s \in D_{n}\right\} .
$$

Tests done for large values of $n$ indicate that the degree distribution is scale-free, see Mon19, Fig. 2 and 3], with $\gamma \approx 1.80$. However, in the largest SCC we only have $\gamma \approx 0.546$. This last LSCC appears because the conjecture can be rewritten as

$$
\frac{4}{m p}=\frac{1}{m x}+\frac{1}{m y}+\frac{1}{m z} \text {, with } p \text { a prime number, }
$$

so the solutions for composites are simply multiples of the solutions associated to their factors.

Concerning the average path length $\langle l\rangle$, for large values of $n$, we have that the average path length $\langle l\rangle$ is smaller than 1, see [Mon19, Fig. 4] because most of the nodes appear in some solution, but the DE is not solved for them.

However, when studying the largest SCC, the value of $\langle l\rangle \approx 2$, see Mon19, Fig. 5]. According to this, the author conjectures that, in the limit, any solution of the DE will probably belong to the LSCC. The author leaves this conjecture open for future lines of research. Finally, another interesting result is the estimation of the clustering coefficient in the largest SCC, which is $C \approx 0.5$.

\subsubsection{Networks based on Fibonacci numbers}

Fibonacci numbers have been used to define iterative networks that can be easily scaled GKD94. These networks permit us to run algorithms that require minimal modifications when we want to adapt them for scaled versions of the network. They have also been for implementing sorting algorithms in parallel computers Sta00. 
However, as we have seen in previous cases, the numerical properties Fibonacci numbers have also been studied from network science. Jing et. al., derive the analytical expressions related to the topology of this network in JJYWGLYCP13. The Fibonacci network built-in time $n$ by $F_{n}$ consists of the genealogy tree genealogy of the first $n$ descendants given by an initial pair of rabbits. Apart from studying the degree distribution, the local clustering coefficient, the average path length, and the average degree $\langle k\rangle$; they also measure the mean time efficiency of random walks on them.
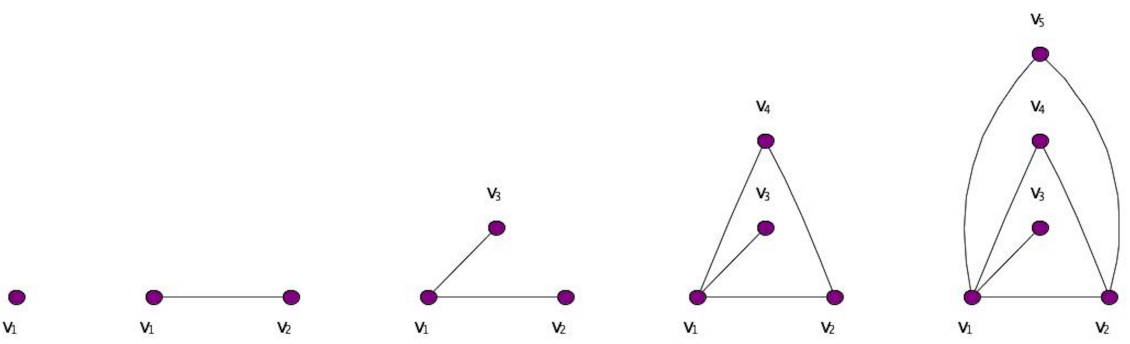

Fig. 1.16: From left to right, Fibonacci Networks $F_{n}$ for times $n=1,2,3,4$, and 5 .

It is important to remark $F_{n}$ is obtained from $F_{n-1}$ recursively. Each network $F_{n}$ will have $n$ nodes. We start with $F_{1}$ with a single node. $F_{2}$ consists of two nodes, $v_{1}$ and $v_{2}$ linked by an edge. Then, $F_{3}$ is generated from $F_{2}$ adding a third node $v_{3}$ and linking them with $v_{1}$ and $v_{2}$. In general, $F_{n}$ is obtained from $F_{n-1}$ adding the $n$-th node $v_{n}$ and connecting them to their ancestors. This yields that the average degree is approximately 2 . To compute the degree distribution, the authors use

$$
p(k)=r_{1}^{-(k+2)}=e^{-(k+2) \alpha} \sim e^{-k \alpha}, \text { with } r_{1}=\frac{1+\sqrt{5}}{2},
$$

where $\alpha=\log \left(r_{1}\right)$. Hence, the degree distribution follows a exponential distribution, like [WKT ${ }^{+}$06, Eq. 9].

The average path length, denoted by $d_{t}$ is computed by

$$
d_{t}=\frac{2 D_{t}}{N_{t}\left(N_{t}-1\right)}
$$

where

$$
D_{t}=\sum_{\substack{i, j \in F_{t} \\ i \neq j}} d_{i j}
$$


here $d_{i j}$ is the are the shortest distance from node $v_{i}$ to node $v_{j}$. It is also worth to mention that the clustering coefficient is zero, because there is no closed triangle. 


\title{
2 Divisibility patterns within Pascal divisibility networks (Journal article)
}

\section{Divisibility patterns within Pascal divisibility networks}

Pedro A. Solares-Hernández, Fernando A. Manzano, Francisco J. PérezBenito, J. Alberto Conejero

Instituto Universitario de Matemática Pura y Aplicada, Universitat Politécnica de Valéncia, Camino de Vera s/n, 46022 Valencia, Spain.

Keywords: network science; divisibility; Pascal matrix.

Complete reference: Solares-Hernández, P.A.; Manzano, F.A.; Pérez-Benito, F.J.; Conejero, J.A. Divisibility Patterns within Pascal Divisibility Networks. Mathematics 2020, 8, 254. https://doi.org/10.3390/math8020254

\begin{abstract}
The Pascal triangle is so simple and rich that it has always attracted the interest of professional and amateur mathematicians. Their coefficients satisfy a myriad of properties. Inspired by the work of Shekatkar et al [SBA15], we study the divisibility patterns within the elements of the Pascal triangle, through its decomposition into Pascal's matrices, from the perspective of network science. Applying Kolmogorov-Smirnov test, we determine that the degree distribution of the resulting network follows a power-law distribution. We also study degrees, global and local clustering coefficients, stretching graph, averaged path length and the mixing assortative.
\end{abstract}

\subsection{Introduction}

Number theory has been one of the most studied fields of mathematics for centuries. In contrast, network science has emerged as a discipline in the last twenty years. Nevertheless, networks have attracted the interest of many researchers due to their multiple applications to different disciplines, such as biology, telecommunications, social and environmental sciences, as well as systems medicine. 
Besides, some networks have emerged from mathematical concepts, such as the divisibility network. This was firstly studied by Zhou et al in [ZWHC06]. Here, nodes represent natural numbers and two nodes $n, m \in \mathbb{N}$ are connected by a directed edge if $n$ divides $m$, denoted by $n \mid m$. These authors noticed that this network has a large clustering coefficient of approximately 0.34, which is insensitive to the network size. Besides, they showed that: (i) the average distance between a pair of nodes is upper bounded, in contrast to small-world networks, (ii) it posses a hierarchical architecture, and (iii) the degree distribution follows a power-law.

A divisibility network can also be considered as a non-directed one if we connect a pair of nodes $a, b \in \mathbb{N}$ if either $a \mid b$ or $b \mid a$. Shekatkar et al [SBA15] studied it using the framework of a growing complex network. Among other properties, they showed that it is scale-free but has a non-stationary degree distribution, reporting a stretching similarity pattern, and showing how this pattern evolves with the size of the network. Related to divisibility networks, Yan et al YWCS16] showed that every layer in a multiplex congruence network is a sparse and heterogeneous subnetwork satisfying the scale-free property, providing an insight into the simultaneous congruences problem through the graphical solutions provided there.

All these results have inspired us to look for other ways of consider the divisibility network through a different growing network procedure. Due to the abundance of beautiful and unpredictable properties hidden in the Pascal Triangle (PT), we have studied the non-directed divisibility following a similar approach as Shekatkar et al did. For this purpose, we have considered squared Pascal symmetric matrices as a covering of growing finite subsets of the PT. These matrices were firstly analyzed by Brawer and Pirovino BP92. We will denote by $S_{n}$ the Pascal square matrix of order $n$, that is obtained when taking the square with two orthogonal sides given by the first $n$ ones of both sides of the PT. As an example, we have indicated in bold font the Pascal matrix $S_{4}$ in Eq. (2.1). From $S_{4}$ we construct a divisibility network whose nodes are $\{1,2,3,4,6,10,20\}$.

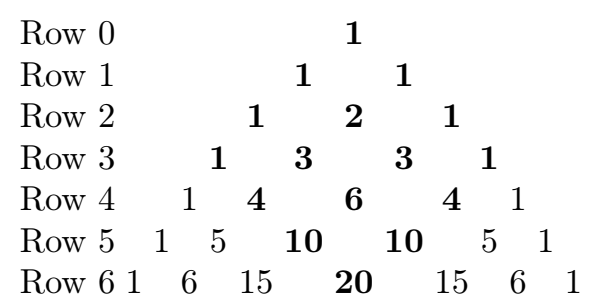

Pascal matrices present some beautiful properties. As an example, we mention the decomposition of the Pascal matrices proposed by Edelman and Strang [ES04 who showed how to decompose any Pascal matrix $S_{n}$ of order $n$, into the product of two matrices $L_{n}$, which is a lower triangular matrix, 
and $U_{n}$, which is an upper triangular matrix, such that, $S_{n}=L_{n} U_{n}$, for every $n \in \mathbb{N}$.

For any given number $k \in \mathbb{N}$, there is some $n_{0} \in \mathbb{N}$ such that $k \in S_{n}$ for all $n \geq n_{0}$. With this in mind, we will study the divisibility networks provided by these matrices. On the one hand, using these matrices, we can cover all the natural numbers through a growing family of subnetworks. On the other hand, the numbers in these matrices are not consecutive, and when sequentially ordered, we can find big gaps between some of them. This suggests us to study whether the scale-free degree distribution holds and other network properties are still satisfied as to the ones shown in [SBA15.

In particular, we have considered the evolution of several network measures along with the size of Pascal matrices, such as the average degree $\langle k\rangle$, the histogram of the connectivity degree distribution; the local and global clustering coefficients $C_{i}$ and $C_{\Delta}$, the assortativity index $r$; and the average path length $\langle d\rangle$. We will recall the definition of these notions in the next section.

The degree distribution is studied in Section 2.2.1, where we show that it is scale-free. We also study an example of how to compute the fitting parameters. In order to study the structure of this network, we have studied how the clustering evolves with the size of the network. The local and global clustering coefficients are presented in Sections 2.2.2 and 2.2.3. The tendency of nodes to connect to nodes of similar degree is analyzed through the assortative coefficient in Section 2.2.4, and how nodes are separated respect to the others through the average path length, see Section 2.2 .5

We refer the reader to the books of Barabási [ $\left.\mathrm{B}^{+} 16\right]$, Estrada [Est12], and Newman [New18, as basic references of Network Science.

\subsection{Network analysis}

For any arbitrary $n \in \mathbb{N}$, we consider the divisibility network $M_{n}=\left(V_{n}, E_{n}\right)$, associated to the Pascal matrix $S_{n}$ of order $n$. This network has $V_{n}$ as its set of nodes and $E_{n}$ as its set of edges. We point out that we exclude the number 1 of $V_{n}$ since it will be linked with any other number, which does not provide useful information for studying the evolution of the network properties of these matrices.

We denote $\operatorname{card}\left(V_{n}\right)$ by $N_{n}$, with $N_{n} \in \mathcal{O}\left(n^{2}\right)$, and $\operatorname{card}\left(E_{n}\right)$ by $L_{n}$. Once fixed the set of nodes $V_{n}$ with the non-repeated elements of $S_{n}$, we recall that an arbitrary pair of elements $a, b \in V_{n}$ are linked by an edge if, and only if, $a \mid b$ or $b \mid a$. Figs. 2.1a and 2.1b are generated from matrices $S_{6}$ and $S_{7}$.

\subsubsection{Degree distribution}

Given the sequence of nodes from a network $M_{n}$, with $n \in \mathbb{N}$, we first analyze the evolution of the degree distribution $p(k)=n_{k} / N_{n}$, where $n_{k}$ is the number 


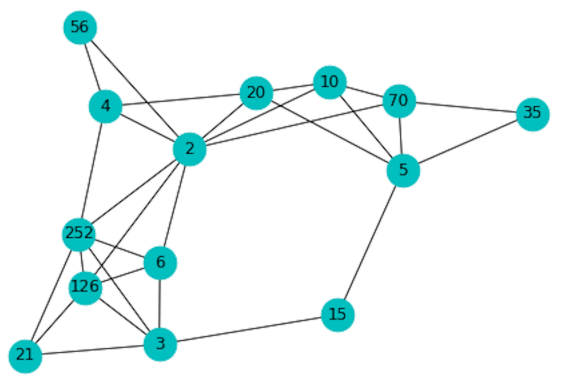

(a) Divisibility network associated to $S_{6}$

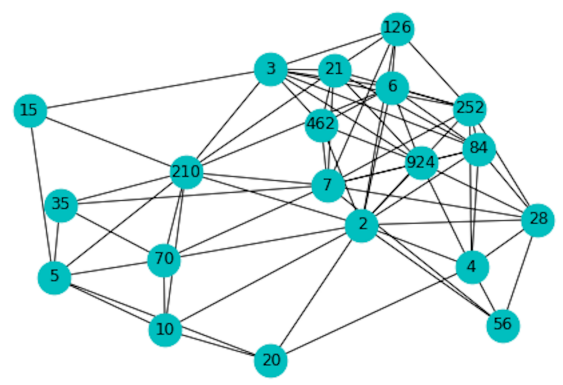

(b) Divisibility network associated to $S_{7}$

Fig. 2.1: Examples of divisibility networks obtained from Pascal matrices $S_{6}$ and $S_{7}$. From $S_{6}$, we have that the network $M_{6}$ has 14 nodes and 29 edges, see Fig. 2.1a), and from $S_{7}$ we have that $M_{7}$ has 20 nodes and 72 edges, see Fig. 2.1b.

of coefficients with degree $k$ in $M_{n}$, along with $n$. First, we check if the scalefree property holds, which will result into the existence of many nodes with only a few edges and a few nodes with a large number of edges, that are called hubs, see $\mathrm{BA} 99, \mathrm{~B}^{+} 16$.

In Fig. 2.2, we illustrate the asymptotic growth of the cumulative and the averaged cumulative degree distributions for the network $M_{2^{6}}$, that has a total of 2001 nodes and 51147 edges. The nodes are indexed in increasing order respect to the term of $S_{2^{6}}$ that they represent. We appreciate how both measures tend to stabilize when adding the last nodes of each network.

Applying the Maximum Likelihood method [CSN09, SBA15, we confirm that the divisibility network of Pascal matrices satisfies a scale-free law. This means that the network degree distribution follows, at least asymptotically, a power-law of the form $p(k)=C \cdot k^{-\gamma}$ for all $k \geq k_{\min }$. We recall that when the power-law governs a process, this usually occurs from what we determinate the "minimum value" $k_{m i n}$, which is exactly the point where one can start to observe the fall of the heavy tail. In Fig. 2.3a, we represent again the degree distribution for $M_{2^{11}}$ with logarithmic binning in the degree values.

We characterize the power-law of the degree distribution of $M_{2^{6}}$ through bootstrapping, using 1000 iterations of bootstrapping. The obtained fitting parameters were $k_{\min }=3$ and $\gamma=1.2256$. In Fig. 22.2a, we plot the histogram of the values obtained for the $\gamma$ parameter. For $\gamma=1.2256$, the KolmogorovSmirnov coefficient was 0.976 , see Fig. $2.3 \mathrm{~b}$, which shows the goodness of the estimation. It is worth to mention that network with similar degree distributions could have a different inner structure, see [Sha12].

We also show the degree distributions of $M_{2^{5}}, M_{2^{6}}, M_{2^{7}}$ and $M_{2^{8}}$ in Fig. 2.4. We observe a high concentration of nodes (plateau) in the region of high values of the degree. This suggests a bias in the linear model fitting due to the 


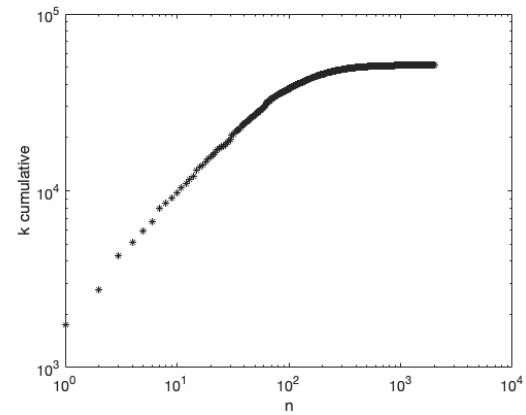

(a)

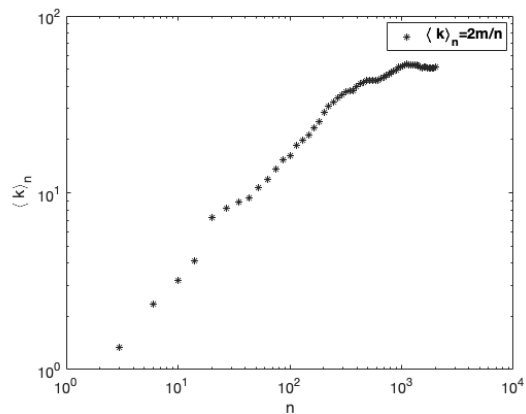

(b)

Fig. 2.2: Cumulative degree and average degree distributions for the network $M_{2^{6}}$. Fig. 2.2a shows the cumulative degree as the coefficients of $S_{2^{6}}$ grow in value. Fig. $2.2 \mathrm{~b}$ plots the evolution of the average degree when the coefficients of $S_{2^{6}}$ are progressively added. For $n=2^{6}$ the average degree is $\langle k\rangle_{n}=51.114$.

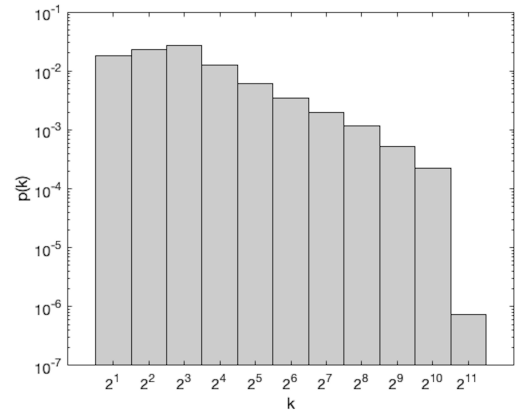

(a)

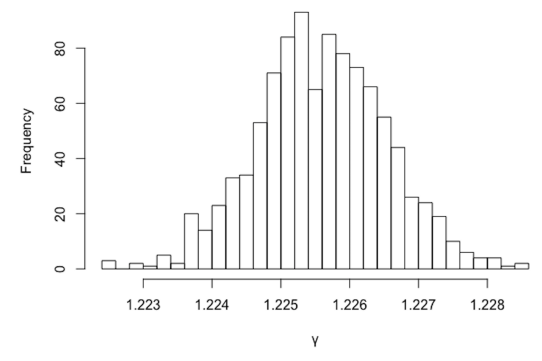

(b)

Fig. 2.3: In Fig. 2.3a, the sizes of the bins are equal to successive positive powers of 2 , and the count in each bin is normalized dividing by the bin width, like in [SBA15, Fig. 2]. In Fig. 2.3b, we characterize the uncertainty in the parameter fitting to a power law using 1000 bootstraps for $M_{2^{6}}$.

comparatively large number of nodes with low degrees respect to the fewer number of nodes with large degrees [B $\mathrm{B}^{+} 16$, Gil15].

In order to correct the non-uniform sampling seen with the linear binning, we show these degrees distributions with logarithmic binning again, see Fig. 2.5 


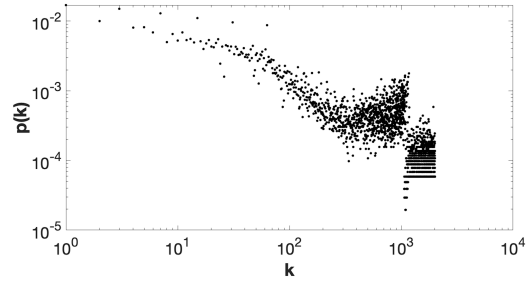

(a) $M_{2^{5}}$

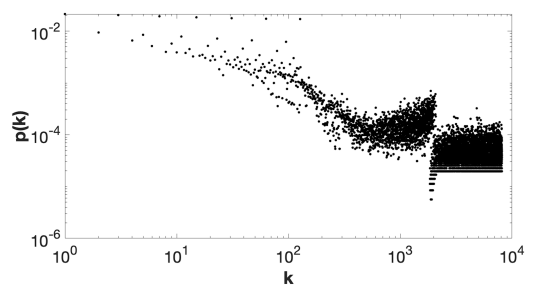

(c) $M_{27}$

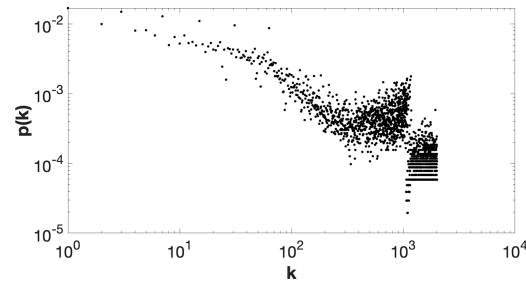

(b) $M_{2^{6}}$

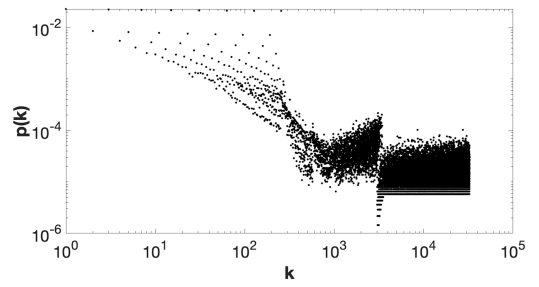

(d) $M_{28}$

Fig. 2.4: Representation of the degree distribution for $M_{2^{5}}, M_{2^{6}}, M_{2^{7}}$, and $M_{2^{8}}$.

As $n$ grows, the $\gamma$ coefficient of the power-law fitting decreases. Increasing the number of nodes of the network does not guarantee a better fit of the linear model. It is worth to mention that these indicators do not fully reflect the network robustness due to the inherent bias of the particular sampling process, see for instance Sha17.

We also have wondered how important is the role of the lowest numbers, which have the highest degrees, in determining the scale-free nature of the network. In this line, we have removed the hubs corresponding to nodes associated with numbers 2 to 6 . These new degree distributions also follow a power-law, as we can see in Fig. 2.6.

\subsubsection{Local clustering coefficient}

Many scale-free networks also display a high degree of clustering. This is the result of a hierarchical organization in which small groups of nodes organize into increasingly larger ones while preserving the scale-free property RB03.

Since all the natural numbers appear along the first row/column of Pascal matrices, and the rest of their elements are composite numbers, there will be many connections from the first elements to the second ones. Besides, as numbers in the inner columns/rows of the matrices grow pretty fast, there will be fewer edges among them. Therefore, we have analyzed how clustered the network is. The main two measures for analyzing the clustering are the 


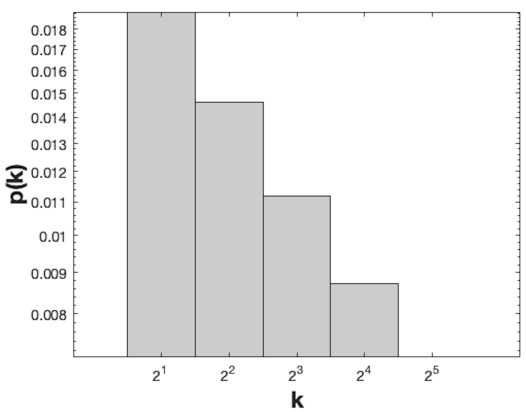

(a) $M_{2^{5}}$

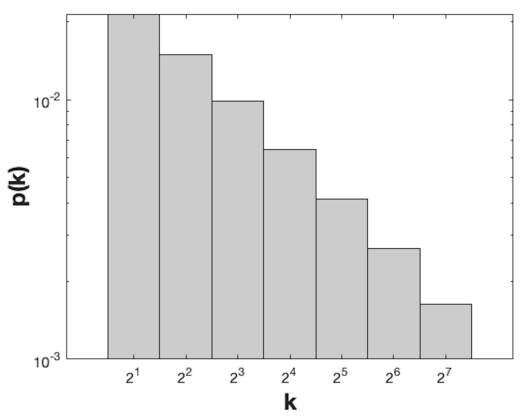

(c) $M_{2^{7}}$

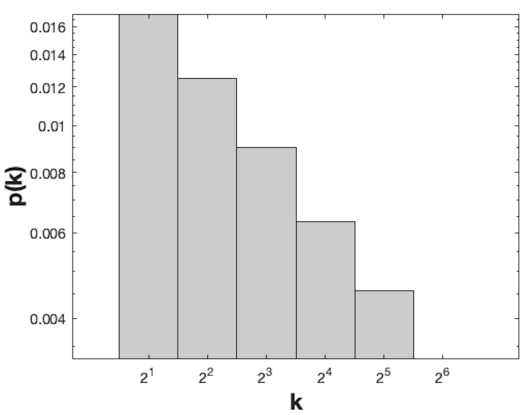

(b) $M_{2^{6}}$

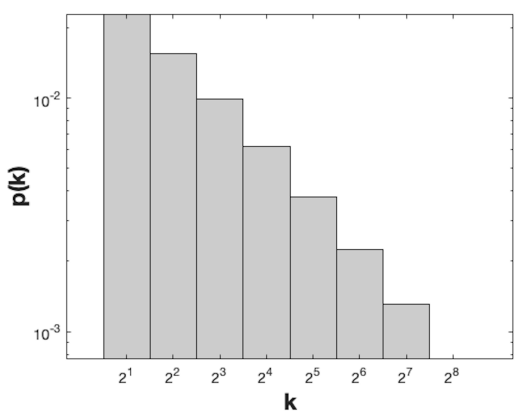

(d) $M_{2^{8}}$

Fig. 2.5: Representation of the degree distribution for $M_{2^{5}}, M_{2^{6}}, M_{2^{7}}$, and $M_{2^{8}}$, with logarithmic bining.

local and the global clustering coefficients. The global version provides an indicator of the clustering in the network, whereas the local one gives an indicator of the connection between the adjacent nodes to a given one.

We recall that given $M_{n}=\left(V_{n}, E_{n}\right)$ and a node $v_{i} \in V_{n}$ with degree $k_{i}$, we denote by $\tilde{v}_{i}$ the set of nodes adjacent to $v_{i}$. Then, the local clustering coefficient $C_{i}$ is the number of pairs of adjacent nodes to $v_{i}$ that are connected between them by an edge, divided by the number of admissible neighbor pairs of $v_{i}$ [DM02, New03b, New18] that is:

$$
C_{i}=\frac{2\left|\left\{l_{j k}: v_{j}, v_{k} \in \tilde{v}_{i}\right\}\right|}{k_{i}\left(k_{i}-1\right)}, \text { where } l_{j k} \text { denotes an edge linking } v_{j} \text { and } v_{k} \text {. }
$$

From Eq. 2.2), we generate some stretching graphs to show how the clustering coefficient evolves when adding the nodes one by one to the networks $M_{2^{6}}, M_{2^{7}}$ and $M_{2^{8}}$, see Fig. 2.7. 


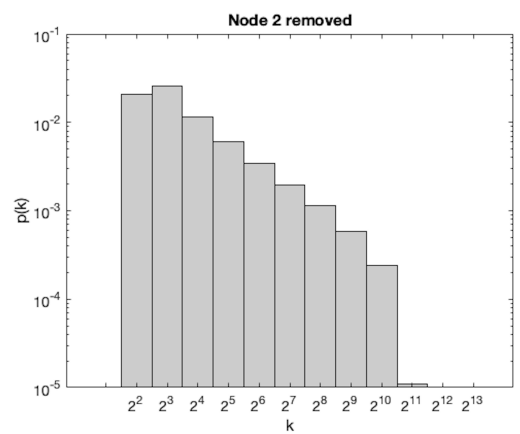

(a)

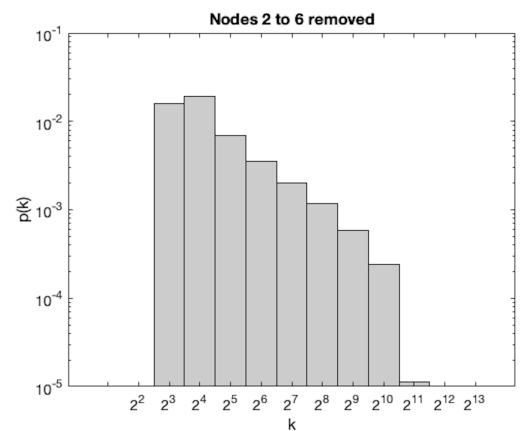

(b)

Fig. 2.6: Degree distributions of the Pascal network $M_{2^{11}}$ with logarithmic bining. In Fig. 2.6a we have removed node 2 and in Fig. 2.6b we have removed nodes 2 to 6 .

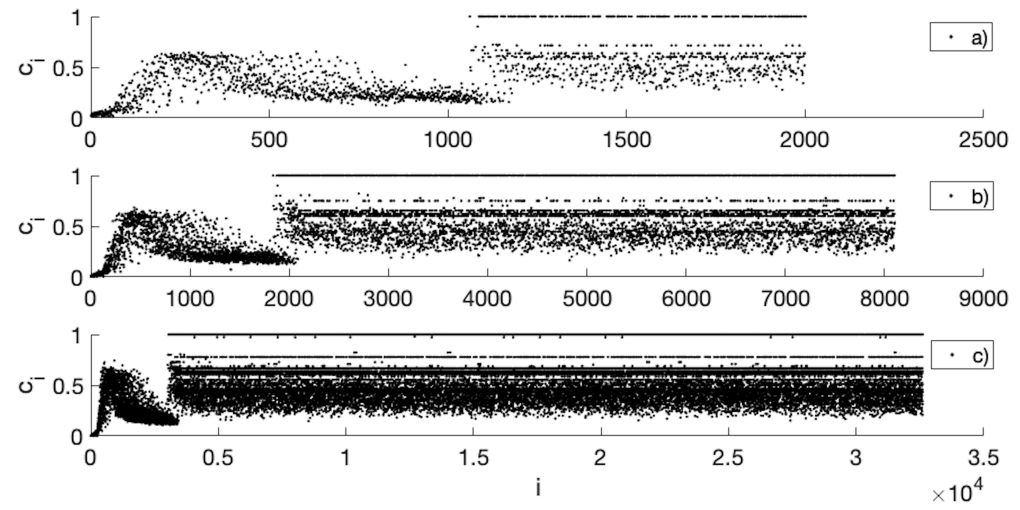

Fig. 2.7: Local clustering coefficient in terms of the node index for different network sizes: (a) $M_{2^{6}}$, (b) $M_{2^{7}}$, (c) $M_{2^{8}}$. Nodes are indexed in increasing order of the number to which they are associated. This graph shows a stretch of similarity due to the divisibility between the coefficients of the Pascal matrix. The stretch is the same, regardless of the size of the network, as it was shown in SBA15.

To see this evolution, we can also represent the difference between the local clustering of two consecutive nodes, namely $\Delta C=C_{i}-C_{i+1}$. This can be compared respect to SBA15, Fig. 5]. Fig. 2.8 shows a symmetrical pattern with respect to the value $\Delta C=0$, as the network grows. 


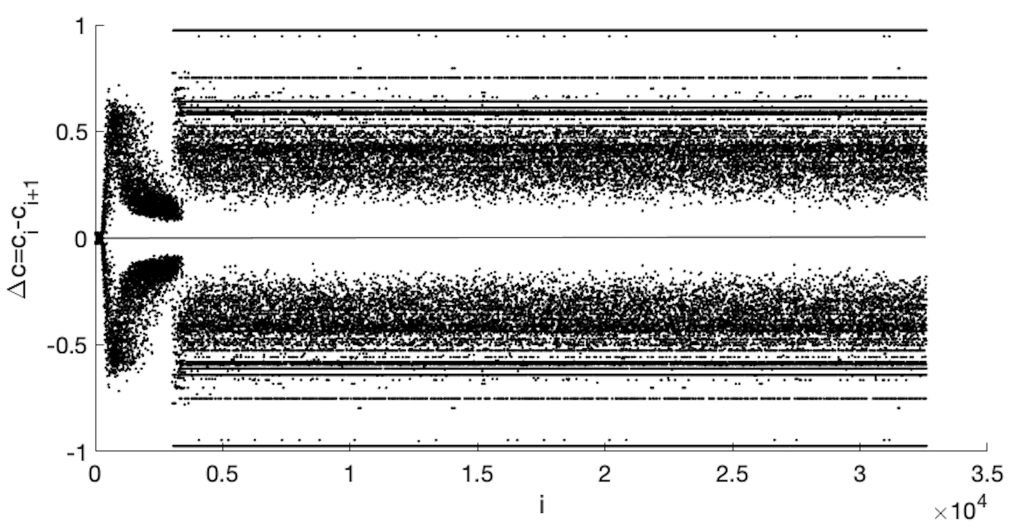

Fig. 2.8: Behavior of the evolution of the difference between the clustering coefficient $C_{i}$ and the clustering coefficient $C_{i+1}$.
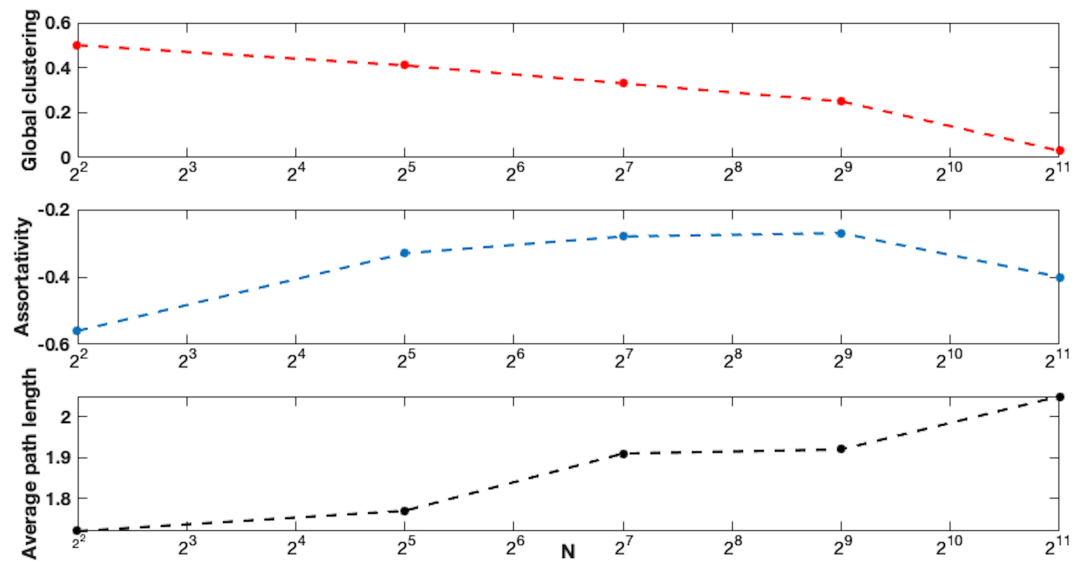

Fig. 2.9: Evolution of the global clustering, assortativity, and average path length for $M_{2^{2}}, M_{2^{5}}, M_{2^{7}}, M_{2^{9}}$, and $M_{2^{11}}$.

\subsubsection{Global clustering coefficient}

The global clustering coefficient is based on ordered triplets of connected nodes, that can be linked by 2 (open triplet) or 3 edges (closed triplet). With this, a triangle graph has 3 closed triplets: That is, a triangle ABC has 3 triplets associated to it: $\mathrm{ABC}, \mathrm{BCA}$, and $\mathrm{CAB}$. With this, the global clustering coefficient can be computed as 


$$
C_{\Delta}=\frac{3 \times \text { number of triangles }}{\text { number of connected triplets }}
$$

As we can see in Fig. 2.9 the global clustering coefficients decreases when the size of $M_{n}$ grows, approaching to 0 for high values of $n$, which represents that the networks $M_{n}$ become more and more sparse when increasing its size $n$.

\subsubsection{Assortative coefficient}

The assortativity coefficient $r$ the correlation coefficient of the degrees of adjacent nodes. It shows the preference of nodes to connect with other nodes of comparable degree New03a, New18].

Given a network $G_{n}=\left(V_{n}, E_{n}\right)$, it is defined as follows:

$$
r=\frac{\sum_{i j}\left(A_{i j}-k_{i} k_{j} / 2 L_{n}\right) k_{i} k_{j}}{\sum_{i j}\left(k_{i} \delta_{i j}-k_{i} k_{j} / 2 L_{n}\right) k_{i} k_{j}}
$$

where $k_{i}$ is the degree of $v_{i}, A_{i j}$ is the $(i, j)^{t h}$ element of the adjacency matrix associated to the eventual connection between $v_{i}$ and $v_{j}, L_{n}$ is the total of links in the network, and $\delta_{i j}$ is the Kronecker delta. However, determining the assortativity from Eq. 3.13 supposes a high computational cost, and therefore, it is suggested to approximate the assortativity by means of the next expression [New18, Sec. 10.7]:

$$
\begin{gathered}
r=\frac{S_{1} S_{e}-S^{2}}{S_{1} S_{3}-S_{2}^{2}} \quad \text { with } \quad S_{e}=\sum_{i j} A_{i j} k_{i} k_{j}=2 \sum_{l_{(i, j)}} k_{i} k_{j}, \text { and } \\
S_{m}=\sum_{i=1}^{N_{n}} k_{i}^{m} \quad \text { for } m=1,2,3, \ldots
\end{gathered}
$$

where we have introduced $l_{(i, j)}$ for referring to all unordered pairs of nodes connected by an edge and $N_{n}$ is the total number of nodes of a network $M_{n}$.

Assortativity coefficient presents values ranging from -1 to 1 . If $r=1$, we called the network to be fully assortative. In case of $r=0$ the network is said to be not assortative, while if $r=-1$, the network is called disassortative [New02, New03a, DM02]. The divisibility networks $M_{n}$ approaches to be disassortative as long as $n$ increases, see Fig. 2.9, which reflects that nodes that have a high degree tend to connect with low-grade nodes.

\subsubsection{Average path length}

The network average path length, denoted by $\langle d\rangle$ is the averaged distance between all pairs of nodes. For each pair, the distance between them is given by the shortest path connecting them. Clearly, if two nodes belong to different 
connected components, the distance will be $\infty$. For computing the average path length we have excluded pairs of nodes belonging to different connected components.

$$
\langle d\rangle=\frac{1}{N(N-1)} \sum_{\substack{i, j=1, N \\ i \neq j}} d_{i, j} .
$$

We can see that, if connected, the averaged path length is short. In Fig. 2.9, we show it for several networks. It increases with the size of the network, but even for $M_{2^{11}}$ it is 2.05 . This means that the biggest connected component is closed to be a bipartite network.

\subsection{Conclusions}

PT and Pascal matrices present multiple properties that have fascinated mathematicians for ages. Here, we have studied them from the perspective of Network Science. We have considered Pascal matrices as a ground for constructing a growing divisibility network. Its structure has been studied, following the approach of [SBA15]. The interest of this choice lies in the fact that PT contains all the natural numbers on each of their sides. The way in which we increasingly construct the divisibility network by taking the Pascal matrices provides a different arrangement of the natural numbers, with gaps between some of them. Nevertheless, the scale-free property for the degree distribution also holds.

Either in [SBA15] or here, both growing networks present similar structures and characteristics to real based networks. This can be noticed when looking at the degree distribution, the global and local clustering coefficients, the assortativity, and the average path length. This work fits within our interest in studying divisibility networks constructed from subsets of the natural numbers, and to see how network measures can help us to describe them and how to find hidden structures and hierarchies BSW19. In future works, we will study the divisibility networks provided by other arrangements of the natural numbers, and the divisibility networks of other countable sets of numbers such as the rational numbers in the unit interval [SHGMC20].

The results concerning the local clustering coefficient are similar to the ones given by [SBA15]. Besides, we have seen that when the size of the network grows, the global clustering coefficient tends to 0 , and the divisibility networks approach more and more to be disassortative. Both results agree with the average path length that, despite low, it increases as the network size grows and indicates that the network is close to be bipartite.

Author Contributions: Conceptualization, PASH. and JAC.; software PASH,FJPB, and FAM; validation and formal analysis, PASH. and JAC; writing-original draft preparation, writing-review and editing, PASH,FJPB, 
FAM, and JAC. All authors have read and agreed to the published version of the manuscript.

Funding: JAC was funded by MEC grant number MTM2016-75963-P. PASH acknowledges the support of MESCyT-RD and Casa Brugal for his $\mathrm{PhD}$ grants.

Conflict of Interest: The authors declare no conflict of interest. The funding institutions had no role in the design of the study; in the collection, analyses, or interpretation of data; in the writing of the manuscript, or in the decision to publish the results. 


\title{
3 Divisibility networks of the rational numbers in the unit interval (Journal article)
}

\section{Divisibility networks of the rational numbers in the unit interval}

Pedro A. Solares-Hernández, Miguel A. García-March, J. Alberto Conejero

Instituto Universitario de Matemática Pura y Aplicada, Universitat Politécnica de Valéncia, Camino de Vera s/n, 46022 Valencia, Spain.

Keywords:network science; complex networks; graph theory; number theory; graphs; abstract algebra.

Complete reference: Solares-Hernández, P.A.; García-March, M.A.; Conejero, J.A. Divisibility Networks of the Rational Numbers in the Unit Interval. Symmetry 2020, 12, 1879. https://doi.org/10.3390/sym12111879

\begin{abstract}
Divisibility networks of natural numbers present a scalefree distribution as many other process in real life due to human interventions. This was quite unexpected since it is hard to find patterns concerning anything related with prime numbers. However, it is by now unclear if this behavior can also be found in other networks of mathematical nature. Even more, it was yet unknown if such patterns are present in other divisibility networks. We study networks of rational numbers in the unit interval where the edges are defined via the divisibility relation. Since we are dealing with infinite sets, we need to define an increasing covering of subnetworks. This requires an order of the numbers different from the canonical one. Therefore, we propose the construction of four different orders of the rational numbers in the unit interval inspired in Cantor's diagonal argument. We motivate why these orders are chosen and we compare the topologies of the corresponding divisibility networks showing that all of them have a free-scale distribution. We also discuss which of the four networks should be more suitable for these analyses.
\end{abstract}




\subsection{Introduction}

Network science is the field that model different phenomena as networks of connected elements. There, the elements are represented by nodes which are connected by links or edges Wat04, PSRDG03, New18, BBV08, $\mathrm{B}^{+} 16$, BFW13, Est12. In recent years this has found applications and received contributions from a wide variety of research fields, such as telecommunications [JD16], machine learning [PBGGNPC20], biology [BO04], social sciences BMBL09, etc.

Recently, network science has been used to study mathematical properties from the point of view of complex systems apart from graph theory itself. Some interesting networks have arisen when studying mathematical structures of numbers sets: divisibility networks of natural numbers following the increasing sequential order, SBA15, divisibility networks of natural numbers according to its arrangement within the Pascal Triangle [SHMPBC20] or networks of prime numbers CD05. Yan et al studied congruence relations through multiplex networks and studied the multiplex congruence network. They found that each one of the layers was sparse and presents a scale-free degree distribution YWCS16.

This motivates our interest in studying how network measures can help us describe them and find hidden structures and hierarchies [BSW19]. In some cases, we can even find analytical expressions of the results, as it is the case of the degree, clustering, geodesic distance, and centrality of the divisibility network on the natural numbers [AA20]. In addition. expressions for other the centrality measures were shown in RA20.

Network science has also provided an approach to analyze conjectures in number theory as it is the case of the Erdös-Straus conjecture Mon19 or the Goldbach conjecture CD05]. Fibonacci networks were studied in JYWWGLYCP13, where the degree distribution and the average path length are calculated. These networks verify the small-world property and have an exponential degree distribution.

In this work, we study divisibility networks where the nodes are rational numbers, each of them connected to other nodes if one divides another one. Despite that we can find rational numbers at a different scale to natural numbers, we wonder if we rational numbers present a symmetric behavior to the natural numbers with respect to the divisibility relation. We also consider that this approach of using network science data analysis tools on datasets of mathematical nature can attract the interest of pure mathematicians to these computational methods.

In principle, these networks consist of infinite sets of numbers (nodes). A common approach is to order the nodes and to define an increasing covering of the set of nodes according to a particular criteria to pick up the nodes. This approach permits to reduce an infinite network to an increasing family of subnetworks whose sets of nodes are in correspondence with the elements of the covering. 
We say that a set $A$ is countable if we can set a bijection $f: \mathbb{N} \rightarrow A$, which, in fact, also yields a sequential order on $A$, taking $a_{1}<a_{2}$, with $a_{1}, a_{2} \in A$, provided that $f^{-1}\left(a_{1}\right)<f^{-1}\left(a_{2}\right)$. Using again bijections, we can prove that the countable union of countable sets is again countable. So, to prove the countability of the rational numbers, it is enough to prove the countability of the rational numbers in the unit interval, and then to consider the countable union of intervals between consecutive integers.

In Section 3.2 we first propose the construction of four different types of networks in the unit interval that are based on Cantor's diagonal argument. Later, we numerically analyze the structure of these networks. In Section 3.3 we study several topological parameters compared to the size of their respective subnetworks, and we see how do they evolve when the size of the network grows. In particular, we discuss the degree distribution $p(k)$; the local clustering coefficient $c(k)$; the average degree $\langle k\rangle_{n}$; the $k$ cumulative degree; the local and global clustering coefficients $C_{i}$ and $C_{\Delta}$; the average clustering $\langle C\rangle$; the assortativity index $r$; the average path length $\langle d\rangle$, and the network density $\rho$. In particular, for the local clustering we represent it splitting the behavior for prime numbers in the numerator and in the numerator. Since the order of the rational numbers does not always correspond with the natural order in the interval, we also indicate their position according to the canonical order and the order obtained through different diagonal arguments. All these measures provide a general overview of the network structure and set the first steps for more detailed analysis that can lead to conjecture analytic formulas that can describe this structure.

Finally, we present the conclusions and draft some future research lines.

\subsection{Matherials and Methods}

For studying the divisibility network of $\mathbb{Q} \cap[0,1]$, we invoke Cantor's diagonal argument to prove its countability Val13]. He arranged all the rational numbers in an infinite-dimensional square matrix and he set a path there to order all their elements sequentially. In this work, we are going to consider several types of these matrices: In the first one, that we will call $\mathcal{A}^{0}=\left(a_{i j}^{0}\right)$, with $a_{i j}^{0}=i / j$ we arrange all positive rational numbers. It is explicitly given by:

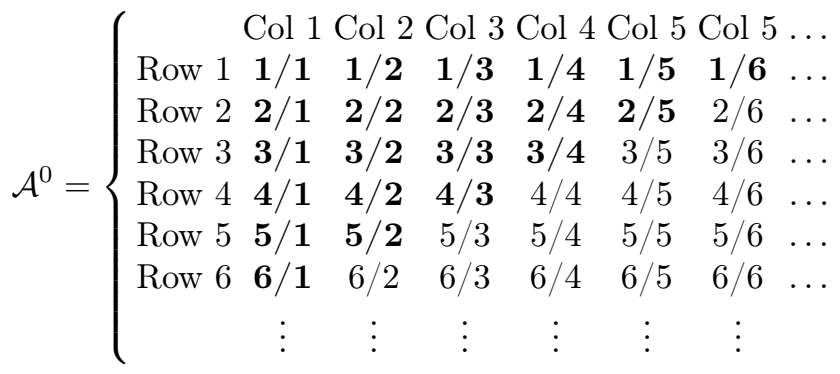


To order them sequentially, one just has to stack one right diagonal after the other. For every $n \in \mathbb{N}$, we define $\mathcal{A}_{n}^{0}$ as the list of numbers extracted from the first $n$-right diagonals of $\mathcal{A}^{0}$. For example, $\mathcal{A}_{6}^{0}$ will be

$$
\mathcal{A}_{6}^{0}=\left(\frac{1}{1}, \frac{1}{2}, \frac{2}{1}, \frac{1}{3}, \frac{2}{2}, \frac{3}{1}, \frac{1}{4}, \frac{2}{3}, \frac{3}{2}, \frac{4}{1}, \frac{1}{5}, \frac{2}{4}, \frac{3}{3}, \frac{4}{2}, \frac{5}{1}, \frac{1}{6}, \frac{2}{5}, \frac{3}{4}, \frac{4}{3}, \frac{5}{2}, \frac{6}{1}\right) .
$$

We start with the divisibility networks that have as the set of nodes all the non repeated elements from $\mathcal{A}_{n}^{0}, n \in \mathbb{N}$. The corresponding set of nodes will be denoted by $V\left(\mathcal{A}_{n}^{0}\right)$. For instance, $V\left(\mathcal{A}_{6}^{0}\right)$ is

$$
V\left(\mathcal{A}_{6}^{0}\right)=\left\{\frac{1}{1}, \frac{1}{2}, \frac{2}{1}, \frac{1}{3}, \frac{3}{1}, \frac{1}{4}, \frac{2}{3}, \frac{3}{2}, \frac{4}{1}, \frac{1}{5}, \frac{5}{1}, \frac{1}{6}, \frac{2}{5}, \frac{3}{4}, \frac{4}{3}, \frac{5}{2}, \frac{6}{1}\right\} .
$$

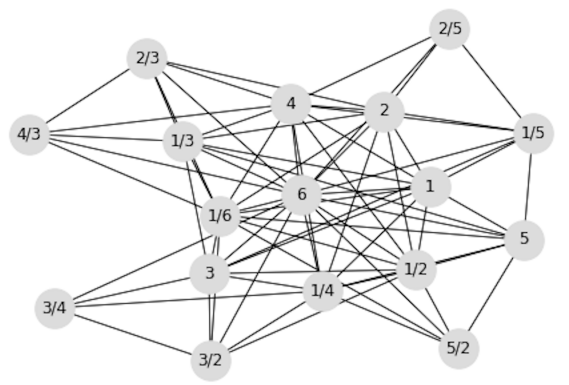

Fig. 3.1: Divisbility network $G\left(\mathcal{A}_{6}^{0}\right)$, with 17 nodes and 71 links.

A connection between nodes, $v_{i}, v_{j} \in V\left(\mathcal{A}_{n}^{0}\right)$ occurs if one divides the other, that is, the division is modulo 0 with a natural number as quotient. We remark that this will provide a non-directed network. We have also considered that 1 is connected with all the numbers, since it is the identity element for the division of non-null elements. We denote as $L\left(\mathcal{A}_{n}^{0}\right)$ the set of links. The networks given by these sets of nodes and links will be denoted by $G\left(\mathcal{A}_{n}^{0}\right)=$ $\left(V\left(\mathcal{A}_{n}^{0}\right), L\left(\mathcal{A}_{n}^{0}\right)\right)$. As an example, we plot in Fig. 3.1 the network $G\left(\mathcal{A}_{6}^{0}\right)$, which has 17 nodes, since $2 / 2$ and $3 / 3$ are equivalent to $1,2 / 4$ is equivalent to $1 / 2$, and $4 / 2$ is equivalent to 2 .

This first network based on Cantor's diagonal argument will inspire the four networks that we will study here. In contrast to $G\left(\mathcal{A}_{n}^{0}\right)$ that is a network of rational numbers, we will consider four different networks, all their elements will belong to $\mathbb{Q} \cap] 0,1]$.

\subsubsection{Networks $G\left(\mathcal{A}_{n}^{1}\right)$}

The first type of network is denoted by $G\left(\mathcal{A}_{n}^{1}\right)=\left(V\left(\mathcal{A}_{n}^{1}\right), L\left(\mathcal{A}_{n}^{1}\right)\right), n \in \mathbb{N}$. It is obtained directly from $\mathcal{A}_{n}^{0}$ by dividing all the nodes by the maximum 
number, which is $n$. This normalization rescales all numbers to fit within the unit interval without affecting the existing links between nodes. For example, from $V\left(\mathcal{A}_{6}^{0}\right)$ one will obtain that the set of nodes $V\left(\mathcal{A}_{6}^{1}\right)$ will be

$$
V\left(\mathcal{A}_{6}^{1}\right)=\left\{\frac{1}{6}, \frac{1}{12}, \frac{1}{3}, \frac{1}{18}, \frac{1}{2}, \frac{1}{24}, \frac{1}{9}, \frac{1}{4}, \frac{2}{3}, \frac{1}{30}, \frac{5}{6}, \frac{1}{36}, \frac{1}{15}, \frac{1}{8}, \frac{2}{9}, \frac{5}{12}, 1\right\} .
$$

\subsubsection{Networks $G\left(\mathcal{A}_{n}^{2}\right)$}

A second network $G\left(\mathcal{A}_{n}^{2}\right)=\left(V\left(\mathcal{A}_{n}^{2}\right), L\left(\mathcal{A}_{n}^{2}\right)\right), n \in \mathbb{N}$, can be obtained from $\mathcal{A}_{n}^{0}$ if we just pick up the numbers in $] 0,1]$ and their connections. Therefore, links between nodes will be also set according to the aforementioned divisibility relation, but the number of nodes and links in $G\left(\mathcal{A}_{n}^{2}\right)$ is smaller than the number of nodes and links of $G\left(\mathcal{A}_{n}^{1}\right)$. For example, the set of nodes with $n=6$ will be

$$
V\left(\mathcal{A}_{6}^{2}\right)=\left\{\frac{1}{1}, \frac{1}{2}, \frac{1}{3}, \frac{1}{4}, \frac{2}{3}, \frac{1}{5}, \frac{1}{6}, \frac{2}{5}, \frac{3}{4}\right\}
$$

which can be compared with $V\left(\mathcal{A}_{6}^{1}\right)$ in Eq. (3.4). For illustrating these definitions, Figs. 3.2 (a) and 3.2 (b) represent $G\left(\mathcal{A}_{6}^{1}\right)$ and $G\left(\mathcal{A}_{6}^{2}\right)$, respectively.

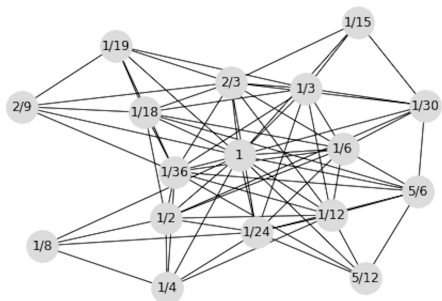

(a)

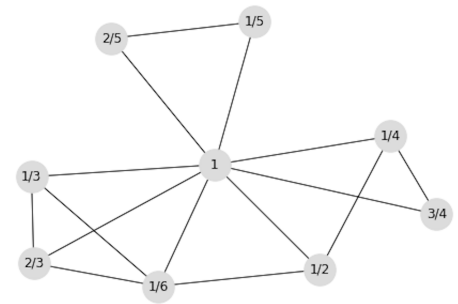

(b)

Fig. 3.2: Divisbility networks $G\left(\mathcal{A}_{6}^{1}\right)$ and $G\left(\mathcal{A}_{6}^{2}\right)$. The first one has 17 nodes and 71 links, and the second one has 9 nodes and 15 links.

\subsubsection{Networks $G\left(\mathcal{B}_{n}^{1}\right)$}

The next two networks are based on a matrix $\mathcal{B}^{0}=\left(b_{i j}^{0}\right)$, with $b_{i j}=i /(j+$ $(i-1))$ and $i, j \in \mathbb{N}$. By construction, this matrix only contains rational numbers in the unit interval. In this matrix, the $n$-right diagonal will have $n$ as a common denominator and the first $n$ natural numbers as numerators. 


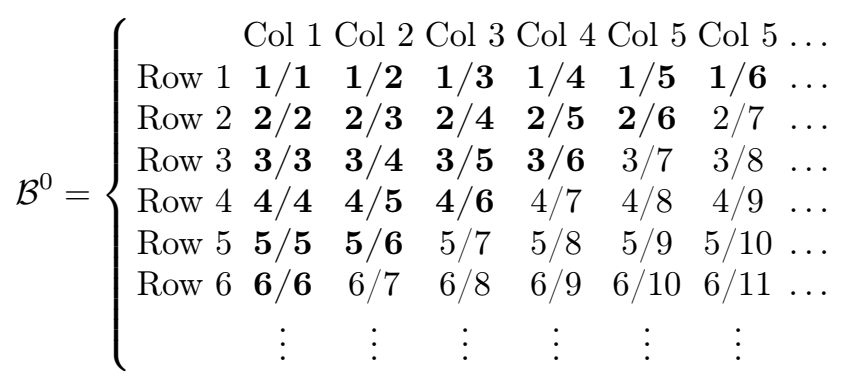

Then, we define $\mathcal{B}_{n}^{0}$ as the set of elements in the first $n$ right diagonals from $\mathcal{B}^{0}$, where $n \in \mathbb{N}$. For example, the elements of $\mathcal{B}_{6}^{0}$ are listed in Eq. (3.7).

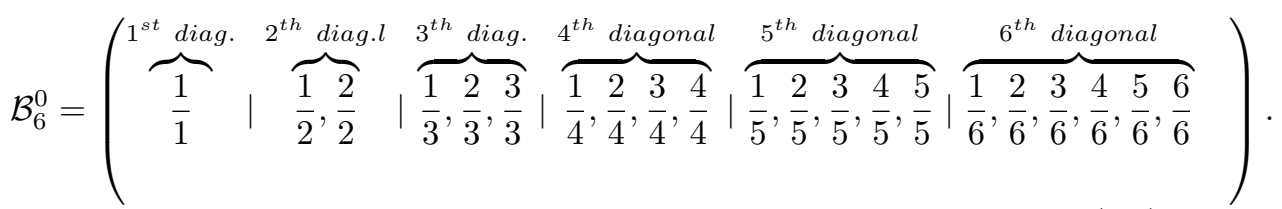

Then, the third type of networks, denoted by $G\left(\mathcal{B}_{n}^{1}\right)=\left(V\left(\mathcal{B}_{n}^{1}\right), L\left(\mathcal{B}_{n}^{1}\right)\right)$, $n \in \mathbb{N}$ will have the non-repeated elements of $\mathcal{B}_{n}^{0}$ as the set of nodes. The set of links will again be given by the divisibility relation. As an example, the set of nodes for $n=6$ is

$$
V\left(\mathcal{B}_{6}^{1}\right)=\left\{\frac{1}{1}, \frac{1}{2}, \frac{1}{3}, \frac{2}{3}, \frac{1}{4}, \frac{3}{4}, \frac{1}{5}, \frac{2}{5}, \frac{3}{5}, \frac{4}{5}, \frac{1}{6}, \frac{5}{6}\right\} .
$$

\subsubsection{Networks $G\left(\mathcal{B}_{n}^{2}\right)$}

Finally, we define a fourth type of network, denoted by $G\left(\mathcal{B}_{n}^{2}\right)=\left(V\left(\mathcal{B}_{n}^{2}\right), L\left(\mathcal{B}_{n}^{2}\right)\right)$, $n \in \mathbb{N}$, whose set of nodes $\mathcal{B}_{n}^{2}$ consists of the elements just in the $n$-th right diagonal of $\mathcal{B}_{k}^{0}$. Again, the sets of links $L\left(\mathcal{B}_{n}^{2}\right)$ is given by the divisibility relation. For example, for $n=6$ one has

$$
V\left(\mathcal{B}_{6}^{2}\right)=\left\{\frac{1}{6}, \frac{2}{6}, \frac{3}{6}, \frac{4}{6}, \frac{5}{6}, \frac{6}{6}\right\} .
$$

We recall that in the first three types of networks, the set of nodes provides a covering of the rational numbers in the unit interval, excluding 0 . However, in this fourth case, the set of nodes $V\left(\mathcal{B}_{n}^{2}\right), n \in \mathbb{N}$ is in correspondence with the first $n$-natural numbers. Note that when running $n$, one obtaines an increasing family of networks isomorphic to the partial subnetworks of the divisibility network of natural numbers, which were analyzed in [SBA15. We represent examples of these last two types of networks in Fig. 3.3, where we plot $G\left(\mathcal{B}_{6}^{i}\right)$ for $i=1,2$. 


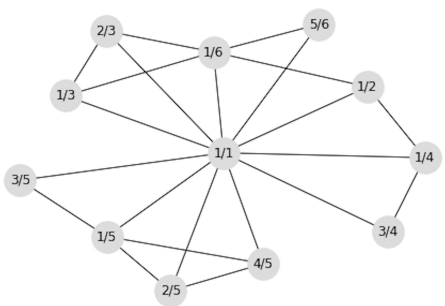

(a)

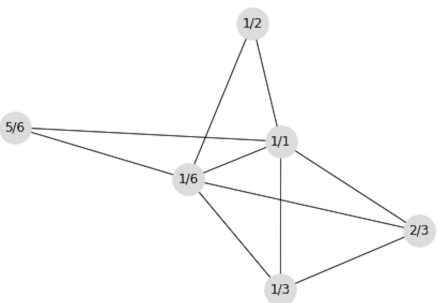

(b)

Fig. 3.3: Divisibility networks $G\left(\mathcal{B}_{6}^{1}\right)$ and $G\left(\mathcal{B}_{6}^{2}\right)$. The first one has 12 nodes and 22 links and the second one has 6 nodes and 10 links.

\subsection{Results}

Since the matrices $\mathcal{A}^{0}$ and $\mathcal{B}^{0}$ are infinite dimensional, we take finite subnetworks of the different four types described above. We start analyzing the complexity of the network through the number of edges. As we have stated previously, despite of considering a similar number of diagonals in matrix $\mathcal{A}^{0}$ and $\mathcal{B}^{0}$, the number of nodes and edges is different for each type of network. For a fixed number of diagonals, the networks $G\left(\mathcal{A}_{n}^{1}\right)$ and $G\left(\mathcal{B}_{n}^{1}\right)$ have more edges and nodes than the respective networks $G\left(\mathcal{A}_{n}^{2}\right)$ and $G\left(\mathcal{B}_{n}^{2}\right)$. The variation of their number of links is a key characteristic that will determine their degree distribution, as the comparative analysis we will carry out will show.

\subsubsection{Degree distribution}

Let us consider an arbitrary network $G=(V, L)$, with $V$ the set of nodes and $L$ the set of edges. We denote by $|V|$ and $|L|$ the cardinals of both sets. We recall that the degree of a node $v \in V$ is the number of links adjacent to $v$. We denote by $p(k), k \in \mathbb{N}$ the frequency with which nodes of degree $k$ appear in $G$. That is, for every $k \in \mathbb{N}$ one has to count how many nodes have degree $k$, which we will denote as $N(k)$, and divide this number by the size of the set of nodes $|V|$, in order to obtain the fraction of nodes in the network with degree $k$, i.e. $p(k)=N(k) /|V|$. This can be illustrated with the histograms of the degree frequency, the cumulative degree, and the average degree $\langle k\rangle=2|L| /|V|$.

In order to better compare the densities we compute a network of each type with $N=2^{16}$ nodes. We plot in Fig. 3.4 the histogram of the degree distribution $p(k)$ with logarithmic bining $\left[\mathrm{B}^{+} 16\right.$, Sec. 4.12 .2$]$ and the logfit to show that the amount of links change very differently for different networks.

These degree distributions are heavy tailed, similarly as it was shown in SBA15, SHMPBC20 for other divisibility networks. These distributions show a plateau for the frequencies of high-order degrees, [ $\left.\mathrm{B}^{+} 16, \mathrm{Ch} .4 .12 .2\right]$. 


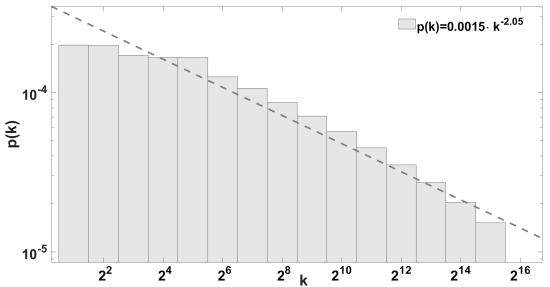

(a)

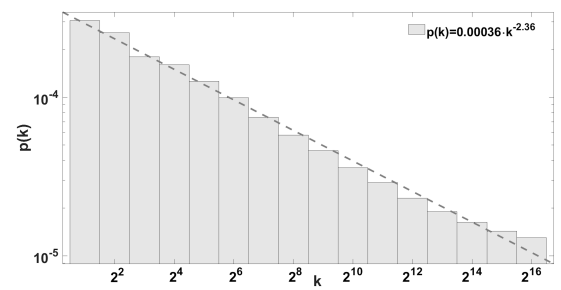

(c)

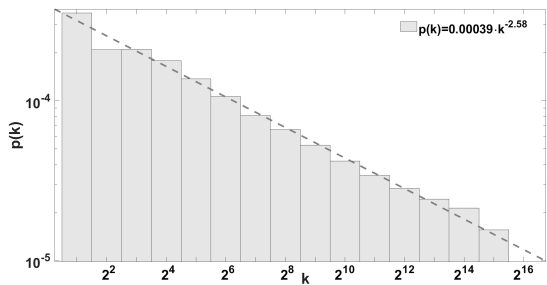

(b)

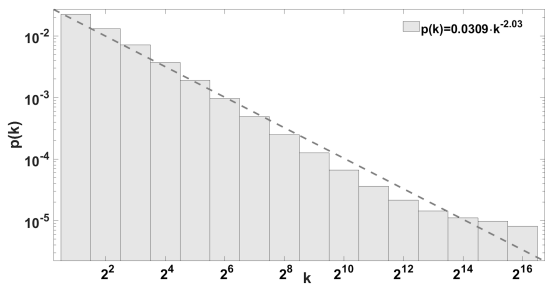

(d)

Fig. 3.4: Degree distribution with log-binning for the networks: (a) $G\left(\mathcal{A}_{n}^{1}\right)$, (b) $G\left(\mathcal{A}_{n}^{2}\right)$, (c) $G\left(\mathcal{B}_{n}^{1}\right)$, (d) $G\left(\mathcal{B}_{n}^{2}\right)$ for a fixed network size of $2^{16}$ nodes.

These distributions can be fitted to a power law distribution of the form $p(k)=C \cdot k^{-\gamma}$, for all $k \geq k_{\min }$. For each type of network we have estimated the values of $k_{\min }$ and $\gamma$ through bootstrapping after 500 iterations. The results for $n=2^{16}$ are shown in Fig. 3.4. On the one hand, the $k_{\text {min }}$ values median are: $k_{\min }\left(G\left(\mathcal{A}_{n}^{1}\right)\right)=4, k_{\min }\left(G\left(\mathcal{A}_{n}^{1}\right)\right)=1, k_{\min }\left(G\left(\mathcal{B}_{n}^{1}\right)\right)=4$, and $k_{\min }\left(G\left(\mathcal{B}_{n}^{2}\right)\right)=2$. We also see that the $\gamma$ estimations for two of the networks are very similar: $\gamma\left(G\left(\mathcal{A}_{n}^{1}\right)\right)=2.05$ and $\gamma\left(G\left(\mathcal{B}_{n}^{2}\right)\right)=2.03$. The other two networks provide higher values of $\gamma$, i.e., $\gamma\left(G\left(\mathcal{A}_{n}^{2}\right)\right)=2.58$ and $\gamma\left(G\left(\mathcal{B}_{n}^{1}\right)\right)=$ 2.36. We recall that for free scale networks, the $\gamma$ of the power-law fitting usually fulfills $2<\gamma<3$, which is satisfied for all the values obtained for $\gamma$. So that, according to these degrees distribution, we have that $G\left(\mathcal{A}^{1}\right)$ is the divisibility network with a more similar behaviour to the divisibility network of the natural numbers, $G\left(\mathcal{B}^{2}\right)$.

Alternatively, we can see how the number of edges increase through the $k$-cumulative degree or the average degree $\langle k\rangle$ in Figs. $3.5 \mathrm{a}$ and $3.5 \mathrm{~b}$ respectively. There are some differences up to $n=10^{3}$. However, from this value, the accumulated degrees tend to behave similarly. We see that network that $G\left(\mathcal{A}^{1}\right)$ presents the highest average degree, and the others present similar values for big network sizes. 


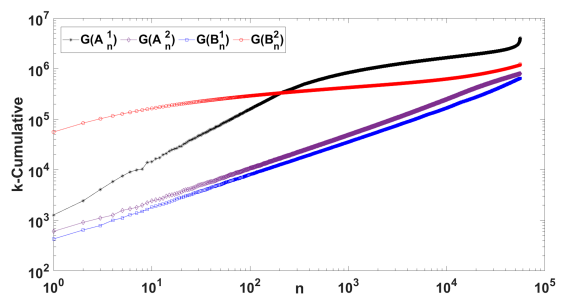

(a)

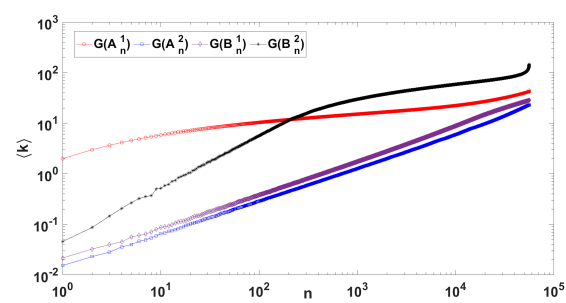

(b)

Fig. 3.5: Evolution of the (a) $k$-cumulative and (b) $\langle k\rangle_{n}$ for the networks $G\left(\mathcal{A}_{n}^{1}\right), G\left(\mathcal{A}_{n}^{2}\right), G\left(\mathcal{B}_{n}^{1}\right)$, and $G\left(\mathcal{B}_{n}^{2}\right)$ for different network sizes up to $2^{16}$ nodes.

\subsubsection{Density and Sparsity}

Given a network $G=(V, L)$, we define its density $\rho(G)$ as the probability of a connection between an arbitrary pair of nodes in $G$. We compute it as the number of edges of the network divided by the maximum admissible number of edges that this network can have. In other words, $\rho(G)=\frac{2|E|}{|V|(|V|-1)}$, see for instance [New18, Ch. 6.10.1]. This value ranges between $0 \leq \rho \leq 1$, the closer to 0 , the more sparse the network is, and the closer to 1 , the more dense it is. For $\rho=0$ we have the null network, and for $\rho=1$ we have a complete network.

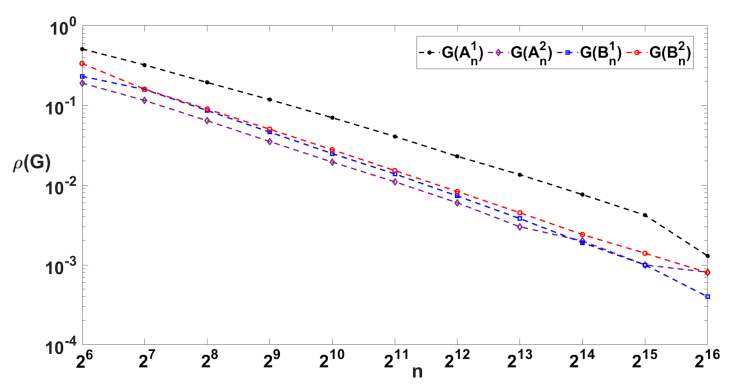

Fig. 3.6: Evolution of the density of networks $G\left(\mathcal{A}_{n}^{1}\right), G\left(\mathcal{A}_{n}^{2}\right), G\left(\mathcal{B}_{n}^{1}\right)$ and $G\left(\mathcal{B}_{n}^{2}\right)$ for different network sizes up to $2^{16}$ nodes.

In Fig. 3.6, we appreciate that the density of $G\left(\mathcal{A}^{1}\right)$ is higher than the others, which agrees with what we observed concerning the average and $k$ cumulative degrees. 


\subsubsection{Local clustering coefficient}

The clustering coefficient can be interpreted as the coefficient that captures the degree to which the neighbors of a given node link to each other $\mathrm{B}^{+} 16$, Ch. 2.10]. Mathematically, the clustering coefficient of a degree $v_{i} \in V$ is computed as:

$$
C_{i}=\frac{2 L_{i}}{k_{i}\left(k_{i}-1\right)},
$$

where $d\left(v_{i}\right)=k_{i}$ and $L_{i} \subset L$ is the set of edges connecting adjacent nodes to $v_{i}$ between them. The local clustering coefficient ranges between 0 and 1 . It can also be understood as the probability that any two adjacent nodes to $v_{i}$ are connected by an edge. This probability gives us information about the density of links in the subnetwork given by the set of nodes adjacent to $v_{i}$. We have represented the stretching separating the cases in which the numerators and denominators are prime or not.

Unlike to the results of [SBA15, SHMPBC20], we have plot the local clustering separating the cases in which numerators (denominators) are prime or not. The nodes are following the order in which they appear following the diagonal argument in their respective matrices. The results are presented in Fig. 3.7. The order in which the nodes appear is represented with colors according to the scale near to each figure. We have also studied if the appearance of prime numbers in the numerator or denominator provide any insight to the clustering coefficient or the similarity stretching, but this is not the case, as we can see in Fig. 3.7. With these networks, the more similar behaviour to $G\left(\mathcal{B}^{2}\right)$ is given by $G\left(\mathcal{A}^{2}\right)$ and $G\left(\mathcal{B}^{1}\right)$ 


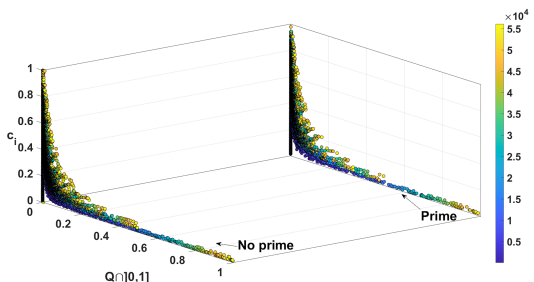

(a)

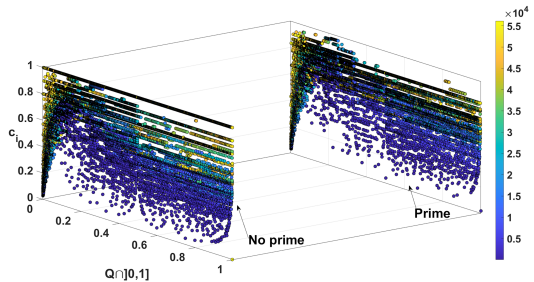

(c)

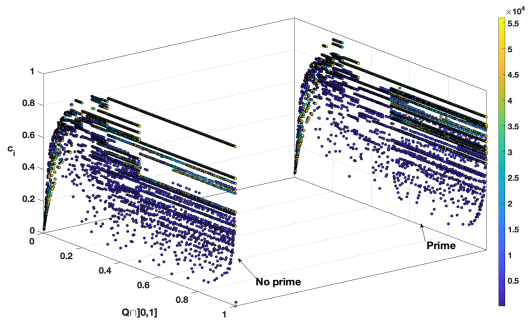

(e)

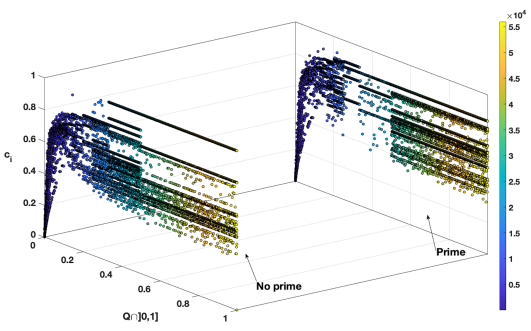

(g)

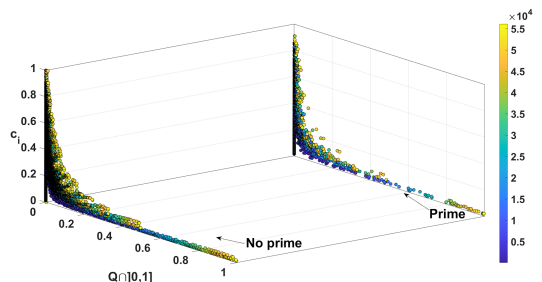

(b)

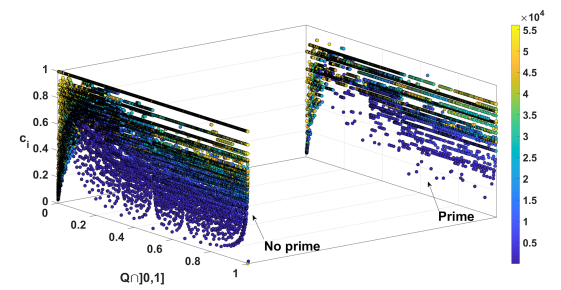

(d)

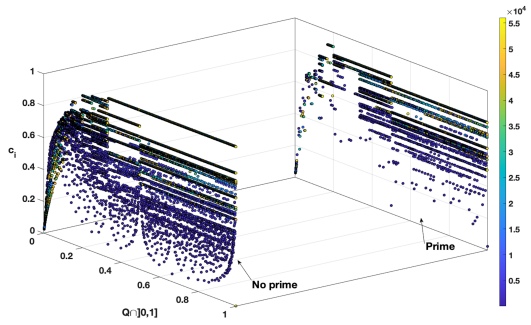

(f)

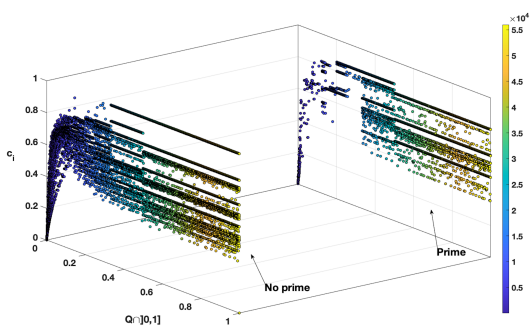

(h)

Fig. 3.7: Local clustering coefficient of the networks (1st row) $G\left(\mathcal{A}_{n}^{1}\right),(2$ nd row) $G\left(\mathcal{A}_{n}^{2}\right),\left(3 \mathrm{rd}\right.$ row) $G\left(\mathcal{B}_{n}^{1}\right)$, and (4t row) $G\left(\mathcal{B}_{n}^{2}\right)$. On the left (right), we separate the values taking into account if the values of the numerator (denominator) are prime or not. Values are colored according to the order of the number following the diagonal argument on the corresponding matrix. 


\subsubsection{Network topologies}

In this section we study four topological parameters: the global clustering coefficient, $C_{\Delta}$, the average clustering coefficient, $\langle C\rangle$, the assortativity coefficient, $r$, and the average path length, $\langle d\rangle$. Further details in [New18, Ch. 6] and $\left[\mathrm{B}^{+} 16\right.$, Ch. 4].

\subsubsection{Global clustering coefficient}

The global clustering coefficient measures the degree of clustering of the whole network. A triplet consist on three nodes of a given network that are connected by edges. If they are just connected by two edges the triplet is said to be open. If they are connected by three, the triplet is closed.

The global clustering coefficient, denoted by $C_{\Delta}$ is the quotient of the total number of closed triplets divided by the total number of triplets (open \& closed).

$$
C_{\Delta}=\frac{3 \times \text { number of closed triplets }}{\text { number of triplets (open \& closed) }} .
$$

We see that it decreases to 0 as the size of the finite subnetworks of each type grow. It can be appreaciated that $C_{\Delta}$ can be approximated by $\frac{1}{N}\left[\mathrm{~B}^{+} 16, \mathrm{Ch}\right.$. 4]. Alternatively, in order to measure the clustering of the whole network one can also study the average clustering coefficient, $\langle C\rangle$, defined as the average of all local clustering coefficients of a network normalized by the network size, that is:

$$
\langle C\rangle=\frac{1}{|V|} \sum_{i=1}^{|V|} C_{i} .
$$

The comparison between both coefficients can be seen in Fig. 3.8. We see that the global clustering coefficient tends to 0 as the size of the network gets bigger for all networks except for $G\left(\mathcal{B}_{n}^{1}\right)$, which seems to stabilize around 0.3. On the contrary, the average clustering coefficient seems to be more inherent to the network and not so influenced by the network size. A significative difference is observed for the values of $G\left(\mathcal{A}_{n}^{1}\right)$ respect to the other networks.

\subsubsection{Assortative coefficient}

In order to measure how nodes are related between them, we compute the Pearson correlation between degrees of adjacent nodes, see for instance New02, New03a, DM02, or the more recent books [New18, Sec. 10.7] and $\left[\mathrm{B}^{+} 16\right]$. The assortative coefficient of a given network is defined in Eq. 3.13).

$$
r=\frac{\sum_{i j}\left(A_{i j}-k_{i} k_{j} / 2|L| k_{i} k_{j}\right.}{\left.\sum_{i j}\right)\left(k_{i} \delta_{i j}-k_{i} k_{j} / 2|L|\right) k_{i} k_{j}}
$$




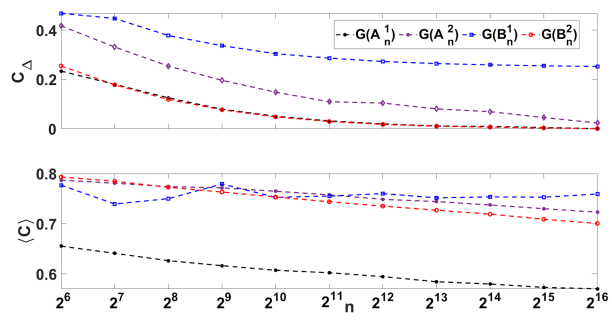

Fig. 3.8: Evolution of the global clustering coeffcient, $C_{\Delta}$, and the average clustering coefficient $\langle C\rangle$, for different network sizes up to $n=2^{16}$.

where $k_{i}$ is the degree of $v_{i}, A_{i j}$ is the $(i, j)^{t h}$ element of the adjacency matrix associated to the eventual connection between $v_{i}$ and $v_{j},|L|$ is the total of links in the network, and $\delta_{i j}$ is the Kronecker delta. However, to determine the assortativity from Eq. 3.13 supposes a high computational cost, and therefore it is suggested to approximate the assortativity by means of the expression

$$
\begin{gathered}
r=\frac{S_{1} S_{e}-S^{2}}{S_{1} S_{3}-S_{2}^{2}} \quad \text { with } \quad S_{e}=\sum_{i j} A_{i j} k_{i} k_{j}=2 \sum_{l_{(i, j)}} k_{i} k_{j}, \quad \text { and } \\
S_{m}=\sum_{i=1}^{N_{n}} k_{i}^{m} \quad \text { for } m=1,2,3, \ldots
\end{gathered}
$$

where we have introduced $l_{(i, j)}$ for referring to all unordered pairs of nodes connected by an edge and $N_{n}$ is the total number of nodes of each one of our networks.

The assortativity coefficient presents values between -1 and 1 . If $r=1$, we called the network to be fully assortative. In case of $r=0$ the network is said to be not assortative, while if $r=-1$, the network is called disassortative. The results can be seen in Fig. 3.9. In the four cases we find a non-assortative pattern that combine with the free-scale property emphasizes that highly connected nodes tend to connect with nodes of low degree.

On the other hand, we observe that the network is dissasortative, $r<$ 1 , and therefore the probability that there are clusters of nodes with the same characteristics is minimal, however, we can see that the assortativity coefficient tends to zero for each network type as $n$ grows. Finally, the average path length $\langle d\rangle \rightarrow 2$ as $n$ grows.

\subsubsection{Average path length}

The network average path length, denoted by $\langle d\rangle$ is the averaged distance between all pairs of different nodes $\left[\mathrm{B}^{+} 16\right]$. For each pair, the distance between 


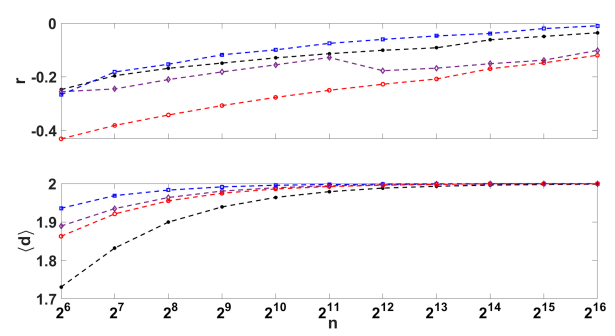

Fig. 3.9: Evolution of the assortativity coefficient, $r$, and the average path length, $\langle d\rangle$, for different network sizes.

them is given by the shortest path connecting them. Since one is connected with all the nodes, then the average path length would be lower than 2 .

$$
\langle d\rangle=\frac{1}{N(N-1)} \sum_{\substack{i, j=1 \\ i \neq j}}^{|V|} d_{i, j} .
$$

In our complex networks, we can see that the $\langle d\rangle \rightarrow 2$ for all networks, which means that all of them are close to be bipartite.

\subsection{Conclusions}

The countability of the set of rational numbers shown by Cantor is one of the most striking elementary results in Mathematics. Starting from his idea of the diagonal arrangement of rational numbers in a matrix, we can propose there is no unique way to set a sequential order in the rational numbers, even in the interval $[0,1]$.

We have explored 4 possible arrangements and their subsequent divisibility networks in order to study divisibility properties from the point of view of Network Science. In all these works, when studying the degree distribution and other network properties, we notice characteristics and structures similar to real based networks.

In all the cases, the obtained results agree with similar results presented by the natural numbers divisibility network explored in [SBA15, SHMPBC20], holding the scale-free property again. We have seen that $G\left(\mathcal{A}^{1}\right)$ behaves in a similar way as $G\left(\mathcal{B}^{2}\right)$. We have also shown how different measures evolve with the network size, and the average path length seems to stabilize in 2, being close to what it yields for a bipartite network. Also, the global clustering coefficient approaches to 0 , except for the case of $G\left(\mathcal{B}_{n}^{1}\right)$, which seems to give a value around 0.3. However, for the rest of the properties, $G\left(\mathcal{A}^{2}\right)$ seems to present more similar characteristics to $G\left(\mathcal{B}^{2}\right)$, the divisibility network of the natural numbers. 
We can find many sequences and arrangements of countable sets in the development of mathematical results, which are susceptible to constructing networks linking them, as we have done here with the divisibility relation. We can also consider other relations among natural or rational numbers that would provide us a network covering of the whole network. We consider of interest to know which are the underlying network topologies in each case. Moreover, we also wonder if we can find some analytical results that would back the obtained results. Besides, we also wonder if this approach can give further insight in order to state and prove new properties of the relation under consideration.

\section{Acknowledgements}

We thank Fernando A. Manzano for supporting us in the computational tasks.

Authors contributions: Conceptualization, PASH, MAGM, JAC.; software computations PASH; validation and formal analysis, PASH, MAGM, and JAC; writing-original draft preparation, writing-review and editing, PASH, MAGM, and JAC. Authors have read and agreed to the published version of the manuscript.

Funding: JAC was funded by MEC grant number MTM2016-75963-P. PASH acknowledges the support of MESCyT-RD, Casa Brugal, and Fundación Proyecto Escuela Hoy Inc. for his PhD grants.

Conflicts of interest: The authors declare no conflict of interest. The funders had no role in the design of the study; in the collection, analyses, or interpretation of data; in the writing of the manuscript, or in the decision to publish the results. 



\section{Discussion of the results and conclusions}

The human brain is an incredible pattern-matching machine.

Jeff Bezos.

With this thesis, we have contributed to describing different properties of Number Theory from the perspective of Network Science by using network representations. We summarize the main conclusions presented in this work and a list of topics to be developed in the future.

\subsection{Discussion of the results}

Network Science emerged as a new discipline motivated by the discovering that most real networks do not behave as random networks since they present hubs, satisfy the small world phenomenon, and present a scale-free degree distribution. Such phenomena have also been noticed in networks generated from mathematical relations, particularly from the divisibility relation in the natural numbers.

We have studied if these properties are satisfied for natural numbers arranged differently, as is the case when we extract an increasing family of Pascal Matrices from the Pascal Triangle. The obtained results can be compared with the ones of [SBA15]. Apart from presenting a scale-free degree distribution, we have seen that the global clustering coefficient approaches 0 , and the network becomes disassortative as the network size grows. Besides, the average path length tends to two, which means that the network tends to be close to a bipartite network.

We have also explored divisibility relation for another countable set, the set of the rational numbers in the unit interval. There is no unique way of setting the bijection to provide a sequential order to these numbers for studying this problem. Therefore, we have studied several configurations inspired by Cantor's diagonal argument to prove the natural numbers' countability. 


\subsection{Future work}

To conclude this memory, we present some research projects that we want to mention some research lines in which we are working and that we want to present some new results in a short future:

1. Divisibility can also provide several different relations in the set of natural numbers that can also be studied by using networks. We can consider that two numbers $a, b \in \mathbb{N}$ are connected if they share a prime factor $p_{i}^{\lambda_{k}}$ with $\lambda_{k} \geq 1$. It will not only be interesting how the properties of these networks when increasing $k$, but also if they are considered as multilayer networks CDARM18. Multilayer networks can be seen as a network with multiple layers. Several configurations can be chosen, either the divisibility by one prime number at each layer or, more generally, increasing the number of common prime factors at each layer.

2. The prime decomposition of natural numbers yields an expression of the total number of divisors of any number directly. Therefore, we can also study the divisibility property from the sequence $\left\{D_{n}\right\}_{n}$, where $D_{n}$ is the number of divisors of $n$. Time series can also be studied with network methods like visibility graphs. Natural and horizontal visibility graphs were introduced by Lacasa et. al., for $\left[\mathrm{LLB}^{+} 08\right.$, LLBL09]. With this approach, we can convert time series into networks, and several properties of the time series are transferred into the network structure. For instance, periodic series are converted into regular networks, random time series into random networks, and fractal series do so into scale-free networks. We want to explore what is the behavior of these networks constructed from the number of divisors.

3. In 1742 Christian Goldbach conjectured that "every pair number greater than 4 is the sum of two prime numbers". Since that time, many mathematicians have tried to solve it, including Goldbach and Euler, but it has not yet been possible, although significant advances have been made in the attempt to prove it.

Chandra and Dasgupta set a network in which each node is a prime number and corresponding to every even number $n=p+q$, with $p, q$ prime numbers, we link $p$, and $q$ with an edge [CD05]. They determined that this network of prime numbers satisfies the small-world property, but the clustering coefficient is high compared to the random network.

However, other approaches can be used to study networks whose generation is based on these conjectures. On the one hand, Schnirelmann demonstrated that there exists a $M$ such that if $n \ggg 2$, starting from a place, $n$ can be represented by, at most, $M$ primes, that is: $n=p_{1}+p_{2}+\cdots+p_{M}$ with $n \ggg 2$ [Sch40]. Later, Wen-Lin proved that $M \leq 18$ [Yin56]. On the other hand, Vinogradov used Schnirelmann's results to demonstrate that if $n$ is a large odd number, then it can be expressed as the sum of three primes: $n=p_{1}+p_{2}+p_{3}$. The most significant approach was 
given by Helfgott, who proved Goldbach's weak conjecture, which states that "every odd number $n$ greater than 5 can be written as the sum of three primes" [Hel13]. It would be interesting to analyze Goldbach's weak conjecture through Network Science and, in particular, to study the clustering in connection to the triplets of numbers used for representing each number bigger than 5 . By now, we already have seen that, applying the Kolmogorov-Smirnov test, the degree distribution behaves similarly to the power-law distribution. 



\section{References}

[AA20] R. Abiya and G. Ambika. Patterns of primes and composites on divisibility graph. arXiv preprint arXiv:2010.12153, 2020.

[AAH02] S.L. Arlinghaus, W.C. Arlinghaus, and F. Harary. Graph theory and geography: An interactive view (eBook). WileyInterscience Series in Discrete Mathematics. John Wiley and Sons, 2002.

[Apo13] T.M. Apostol. Introduction to analytic number theory. Springer Science \& Business Media, 2013.

[Ara10] J.R. Arana. La idea de infinito en la filosofía de Descartes. Ontology studies, pages 131-142, 2010.

[Arm98] M. Armstrong. Network interconnection in telecommunications. Econ. J., 108(448):545-564, 1998.

[B $\left.{ }^{+} 16\right]$ A.L. Barabási et al. Network science. Cambridge University Press, 2016.

[BA99] A.L. Barabási and R. Albert. Emergence of scaling in random networks. Science, 286(5439):509-512, 1999.

$\left[\mathrm{BBB}^{+} 14\right]$ N. Boers, B. Bookhagen, H.M.J. Barbosa, N. Marwan, J. Kurths, and J.A. Marengo. Prediction of extreme floods in the eastern Central Andes based on a complex networks approach. Nat. Comm., 5(1):1-7, 2014.

$\left[\mathrm{BBK}^{+} 12\right]$ A. Bashan, R.P. Bartsch, J.W. Kantelhardt, S. Havlin, and P.Ch. Ivanov. Network physiology reveals relations between network topology and physiological function. Nat. Comm., 3(1):1-9, 2012.

[BBV08] A. Barrat, M. Barthelemy, and A. Vespignani. Dynamical processes on complex networks. Cambridge university press, 2008.

[BF02] A.L. Barabási and J. Frangos. Linked: the new science of networks science of networks. Basic Books, 2002.

[BFW13] U. Brandes, L.C. Freeman, and D. Wagner. Social networks. CRC Press, 2013. 
[BMBL09] S.P. Borgatti, A. Mehra, D.J. Brass, and G. Labianca. Network analysis in the social sciences. Science, 323(5916):892-895, 2009.

[BO04] A.L. Barabasi and Z.N. Oltvai. Network biology: understanding the cell's functional organization. Nat. Rev. Genet., 5(2):101-113, 2004.

[Bor66] Z.I. Borevich. Number theory. Pure and applied mathematics. Academic Press, New York, [electronic resource] edition, 1966.

[BP92] R. Brawer and M. Pirovino. The linear algebra of the Pascal matrix. Linear Algebr. its Appl., 174:13-23, 1992.

[BSW19] Leonid Bunimovich, Dallas Smith, and Benjamin Webb. Finding hidden structures, hierarchies, and cores in networks via isospectral reduction. Appl. Math. Nonlinear Sci., 4(1):225-248, 2019.

[CD05] A.K. Chandra and S. Dasgupta. A small world network of prime numbers. Physica A, 357(3-4):436-446, 2005.

[CDARM18] E. Cozzo, Gu.F. De Arruda, F.A. Rodrigues, and Y. Moreno. Multiplex networks: basic formalism and structural properties. Springer, 2018.

[CH03] R. Cohen and S. Havlin. Scale-free networks are ultrasmall. Phys. Rev. Let., 90(5):058701, 2003.

[CHBA03] R. Cohen, S. Havlin, and D. Ben-Avraham. Efficient immunization strategies for computer networks and populations. Phys. Rev. Let., 91(24):247901, 2003.

[Cor04] G. Corso. Families and clustering in a natural numbers network. Phys. Rev. E, 69(3):036106, 2004.

$\left[\mathrm{CPH}^{+} 08\right]$ Y. Chen, G. Paul, S. Havlin, F. Liljeros, and H.E. Stanley. Finding a better immunization strategy. Phys. Rev. Let., 101(5):058701, 2008.

[CSN09] A. Clauset, C.R. Shalizi, and M.E.J. Newman. Power-law distributions in empirical data. SIAM Rev., 51(4):661-703, 2009.

[Dau90] J.W. Dauben. Georg Cantor: His mathematics and philosophy of the infinite. Princeton University Press, 1990.

[DGGB11] Charo I Del Genio, Thilo Gross, and Kevin E Bassler. All scale-free networks are sparse. Physical review letters, 107(17):178701, 2011.

[DM02] S.N. Dorogovtsev and J.F.F. Mendes. Evolution of networks. Adv. Phys., 51(4):1079-1187, 2002.

[DSPK78] I. De Sola Pool and M. Kochen. Contacts and influence. Soc. Networks, 1(1):5 - 51, 1978.

[EA75] S.F. Edwards and P.W. Anderson. Theory of spin glasses. J. Phys. F Metal Phys., 5(5):965-974, May 1975. 
[ER59] P. Erdös and A. Renyi. On random graphs I. Publ. Math., pages 290-297, 1959.

[ER60] P. Erdös and A. Rényi. On the evolution of random graphs. Publ. Math. Inst. Hung. Acad. Sci, 5(1):17-60, 1960.

[ES04] A. Edelman and G. Strang. Pascal matrices. Amer. Math. Mon., 111(3):189-197, 2004.

[ES13] F. Emmert-Streib. Structural properties and complexity of a new network class: Collatz step graphs. PLoS ONE, 8(2):e56461, 2013.

[ESG11] F. Emmert-Streib and G.V. Glazko. Network biology: a direct approach to study biological function. Wiley Interdiscip. Rev. Syst. Biol. Med., 3(4):379-391, 2011.

[Est12] E. Estrada. The structure of complex networks: theory and applications. Oxford University Press, 2012.

$\left[\mathrm{FMC}^{+} 16\right]$ J.F. Fan, J. Meng, X.S. Chen, Y. Ashkenazy, and S. Havlin. Network approaches to climate science. Sci. China Phys. Mech., 60(1):010531, Oct 2016.

$\left[\mathrm{GGB}^{+} 12\right]$ O. Guez, A. Gozolchiani, Y. Berezin, S. Brenner, and S. Havlin. Climate network structure evolves with north atlantic oscillation phases. EPL (Europhysics Letters), 98(3):38006, 2012.

[Gil59] E.N. Gilbert. Random graphs. Annals Math. Stat., 30(4):1141-1144, 1959.

[Gil15] C.S. Gillespie. Fitting heavy tailed distributions: The poweRlaw package. J. Stat. Softw., 64(2):1-16, 2015.

[GKD94] R. K Govindaraju, M.S. Krishnamoorthy, and N. Deo. Fibonacci networks. Fibonacci Q., 32(4):329, 1994.

[GPSB14] G. Garcia-Perez, M. A. Serrano, and M. Boguná. Complex architecture of primes and natural numbers. Phys. Rev. E, 90(2):022806, 2014.

[HC18] S.G. Hofmann and J. Curtiss. A complex network approach to clinical science. Eur. J. Clin. Invest., 48(8):e12986, 2018.

[Hel13] H.A. Helfgott. The ternary goldbach conjecture is true. arXiv preprint arXiv:1312.7748, 2013.

[HHLM99] L.H. Hartwell, J.J. Hopfield, S. Leibler, and A.W. Murray. From molecular to modular cell biology. Nature, 402(6761):C47-C52, 1999.

[Hop82] J.J. Hopfield. Neural networks and physical systems with emergent collective computational abilities. Proc. Natl. Acad. Sci. U.S.A., 79(8):2554-2558, 1982.

[JD16] F. Jian and S. Dandan. Complex network theory and its application research on p2p networks. Appl. Math. Nonlinear Sci., 1(1):45-52, 2016. 
[JDGDM14] S. Johnson, V. Domínguez-García, L. Donetti, and M.A. Muñoz. Trophic coherence determines food-web stability. Proc. Natl. Acad. Sci. U.S.A., 111(50):17923-17928, 2014.

[JTMM10] S. Johnson, J.J. Torres, J. Marro, and M.A. Munoz. Entropic origin of disassortativity in complex networks. Phys. Rev., 104(10):108702, 2010.

[JYWGLYCP13] Z. Jing-Yuan, S. Wei-Gang, T. Li-Yan, and L. Chang-Pin. Topological properties of Fibonacci networks. Commun. Number Theory Phys., 60(3):375, 2013.

[Koc89] M. Kochen. The small world. Ablex Pub., 1989.

[Lag85] J.C. Lagarias. The $3 x+1$ problem and its generalizations. Amr. Math. Month., 92(1):3-23, 1985.

[LBHGSR21] A. Lampo, J.r Borge-Holthoefer, S. Gómez, and A. SoléRibalta. Multiple abrupt phase transitions in urban transport congestion. Phys. Rev. Res., 3:013267, Mar 2021.

$\left[\mathrm{LEA}^{+}\right.$01] F. Liljeros, C.R. Edling, L.A.N. Amaral, H.E. Stanley, and Y. Åberg. The web of human sexual contacts. Nature, 411(6840):907-908, 2001.

$\left[\mathrm{LFW}^{+} 15\right]$ D. Li, B. Fu, Y. Wang, G. Lu, Y. Berezin, H.E.e Stanley, and S. Havlin. Percolation transition in dynamical traffic network with evolving critical bottlenecks. Proc. Natl. Acad. Sci. U.S.A., 112(3):669-672, 2015.

$\left[\mathrm{LLB}^{+} 08\right]$ L. Lacasa, B. Luque, F. Ballesteros, J. Luque, and J.C. Nuño. From time series to complex networks: The visibility graph. Proc. Natl. Acad. Sci. USA, 105(13):4972-4975, 2008.

[LLBL09] B. Luque, L. Lacasa, F. Ballesteros, and J. Luque. Horizontal visibility graphs: Exact results for random time series. Phys. Rev. E, 80(4):046103, 2009.

[LML08] B. Luque, O. Miramontes, and L. Lacasa. Number theoretic example of scale-free topology inducing self-organized criticality. Phys. Rev. Let., 101(15):158702, 2008.

[Loc09] P. Lockhart. A Mathematician's Lament: How school cheats us out of our most fascinating and imaginative art form. Bellevue Literary press, 2009.

[Lor95] E.N. Lorenz. The essence of chaos. University of Washington Press, 1995.

$\left[\mathrm{LSD}^{+}\right.$20] Y.Y. Liu, H. Sanhedrai, G.G. Dong, F. Shekhtman, L.M .and Wang, S.V. Buldyrev, and S. Havlin. Efficient network immunization under limited knowledge. Natl. Sci. Rev., 8(1), 09 2020. nwaa229.

[Man82] B.B. Mandelbrot. The fractal geometry of nature, volume 1. WH freeman New York, 1982. 
[MBMK12] N. Malik, B. Bookhagen, N. Marwan, and J. Kurths. Analysis of spatial and temporal extreme monsoonal rainfall over South Asia using complex networks. Clim. Dyn., 39(34):971-987, 2012.

[Mil67a] S. Milgram. Six degrees of separation. Psychol. Today, 2:60-64, 1967.

[Mil67b] S. Milgram. The small world problem. Psychol. Today, 2(1):60-67, 1967.

[Mon19] V. Mondreti. A complex networks approach to analysing the Erdös-Straus conjecture and related problems. Preprint, 2019.

[MT69] S. Milgram and J. Travers. An experimental study of the small world problem. Sociometry, 32(4):425-443, 1969.

[New01] M E.J. Newman. The structure of scientific collaboration networks. Proc. Natl. Acad. Sci. U.S.A., 98(2):404-409, 2001.

[New02] M.E.J. Newman. Assortative mixing in networks. Phys. Rev., 89(20):208701, 2002.

[New03a] M.E.J. Newman. Mixing patterns in networks. Phys. Rev., 67(2):026126, 2003.

[New03b] M.E.J. Newman. The structure and function of complex networks. SIAM Rev., 45(2):167-256, 2003.

[New05] M.E.J. Newman. Power laws, Pareto distributions and Zipf's law. Contemp. Phys., 46(5):323-351, 2005.

[New18] M.E.J. Newman. Networks. Oxford university press, 2018.

$\left[\mathrm{OSH}^{+} 07\right]$ J.P. Onnela, J. Saramäki, J. Hyvönen, G. Szabó, D. Lazer, K. Kaski, J. Kertész, and A.L. Barabási. Structure and tie strengths in mobile communication networks. Proc. Natl. Acad. Sci. U.S.A., 104(18):7332-7336, 2007.

[PBGGNPC20] F. Javier Pérez-Benito, J.M. García-Gómez, E. NavarroPardo, and J.A. Conejero. Community detection-based deep neural network architectures: A fully automated framework based on likert-scale data. Math. Methods Appl. Sci., 43(14):8290-8301, 2020.

[PLC91] S.L. Pimm, J.H. Lawton, and J.E. Cohen. Food web patterns and their consequences. Nature, 350(6320):669-674, 1991.

[PSRDG03] R. Pastor-Satorras, M. Rubi, and A. Diaz-Guilera. Statistical mechanics of complex networks, volume 625 . Springer Science \& Business Media, 2003.

[PSV01] R. Pastor-Satorras and A. Vespignani. Epidemic spreading in scale-free networks. Phys. Rev. Let., 86(14):3200, 2001. 
[RA20] A. Rajans and G. Ambika. Patterns of primes and composites from divisibility network of natural numbers. arXiv preprint arXiv:2007.00769, 2020.

[RB03] E. Ravasz and A.L. Barabási. Hierarchical organization in complex networks. Phys. Rev., 67(2):026112, 2003.

[Ros13] M. Rosen. Number theory in function fields, volume 210. Springer Science \& Business Media, 2013.

$\left[\mathrm{RSM}^{+} 02\right]$ E. Ravasz, A.L. Somera, D.A. Mongru, Z.N. Oltvai, and A.L. Barabási. Hierarchical organization of modularity in metabolic networks. Science, 297(5586):1551-1555, 2002.

[SBA15] S.M. Shekatkar, C. Bhagwat, and G. Ambika. Divisibility patterns of natural numbers on a complex network. Sci. Rep., 5:14280, 2015.

[Sch40] L.G. Schnirelman. Prime numbers. Gosudarstvenoe izdateljstvo tehnikoteoreticheskoi literaturi (Russian), Moskva-Leningrad, 1940.

[SEM18] M. Saleh, Y. Esa, and A. Mohamed. Applications of complex network analysis in electric power systems. Energies, 11(6):1381, 2018.

[Sha12] Y. Shang. Distinct clusterings and characteristic path lengths in dynamic small-world networks with identical limit degree distribution. J. Stat Phys., 149(3):505-518, 2012.

[Sha17] Y. Shang. Subgraph robustness of complex networks under attacks. IEEE Trans. Syst. Man Cybern. Syst., 49(4):821832, 2017.

[SHGMC20] P.A. Solares-Hernández, M.A. García-March, and J.A. Conejero. Divisibility networks of the rational numbers in the unit interval. Symmetry, 12(11):1879, 2020.

[SHMPBC20] P.A. Solares-Hernández, F.A. Manzano, F.J. Pérez-Benito, and J.A. Conejero. Divisibility patterns within Pascal divisibility networks. Mathematics, 8(2):254, 2020.

[SL84] M.R. Schroeder and R.B. Lindsay. Number Theory in Science and Communication, With Applications in Cryptography, Physics, Biology, Digital Information and Computing. Acoustical Society of America, 1984.

[SR51] R. Solomonoff and A. Rapoport. Connectivity of random nets. Bull. Math. Biophys., 13(2):107-117, 1951.

[Sta00] G. Stachowiak. Fibonacci correction networks. In Algorithm Theory - SWAT 2000, 7th Scandinavian Workshop on Algorithm Theory, pages 535-548. Springer, 2000.

[Str12] S.H. Strogatz. The Joy of $x$ : a Guided Tour of Math, from one to infinity. Houghton Mifflin Harcourt, 2012. 
[Val13] R.W. Vallin. The elements of Cantor sets: with applications. Wiley Online Library, 2013.

[VBH20] L.D. Valdez, L.A. Braunstein, and S. Havlin. Epidemic spreading on modular networks: The fear to declare a pandemic. Phys. Rev. E, 101(3):032309, 2020.

[Wat04] D.J. Watts. Small worlds: the dynamics of networks between order and randomness, volume 9. Princeton University Press, 2004.

[Wea96] D.B. West and et al. Introduction to graph theory, volume 2. Prentice Hall Upper Saddle River, NJ, 1996.

[Wil75] K.G. Wilson. The renormalization group: Critical phenomena and the kondo problem. Rev. Mod. Phys., 47(4):773, 1975.

$\left[\mathrm{WKT}^{+} 06\right]$ D.R. White, N. Kejžar, C. Tsallis, D. Farmer, and S. White. Generative model for feedback networks. Phys. Rev. E, 73(1):016119, 2006.

[WS98] D.J. Watts and S.H. Strogatz. Collective dynamics of'small-world'networks. Nature, 393(6684):440-442, 1998.

$\left[\mathrm{WWA}^{+} 08\right]$ L. Wu, B.N. Waber, S. Aral, E. Brynjolfsson, and A. Pentland. Mining face-to-face interaction networks using sociometric badges: Predicting productivity in an it configuration task. SSRN, (1130251), 2008.

[Yin56] Wen-lin Yin. Note on the representation of large integers as sums of primes. Bull. Acad. Polon. Sci., 16:793-795, 1956.

[YWCS16] X.Y. Yan, W.X. Wang, G.R. Chen, and D.H. Shi. Multiplex congruence network of natural numbers. Sci. Rep., 6:23714, 2016.

[YZW $\left.{ }^{+} 14\right]$ Z. Yuan, C. Zhao, W.-X. Wang, Z. Di, and Y.-C. Lai. Exact controllability of multiplex networks. New J. Physics, 16(10):103036, 2014.

[Zac77] W.W. Zachary. An information flow model for conflict and fission in small groups. Journal of anthropological research, 33(4):452-473, 1977.

[Zha09] A. Zhang. Protein interaction networks: computational analysis. Cambridge University Press, 2009.

[ZWHC06] T. Zhou, B.-H. Wang, P.M. Hui, and K.P. Chan. Topological properties of integer networks. Physica A, 367:613-618, 2006. 